\section{NRTSC}

Nuclear Reactor Technology And Scientific Computations
NRTSC

Technology

On Time

On Target

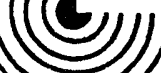

\title{
Statistical Analysis of Test Data for APM Rod Issue (U)
}

\author{
by \\ T. B. Edwards \\ S. P. Harris \\ C. P. Reeve

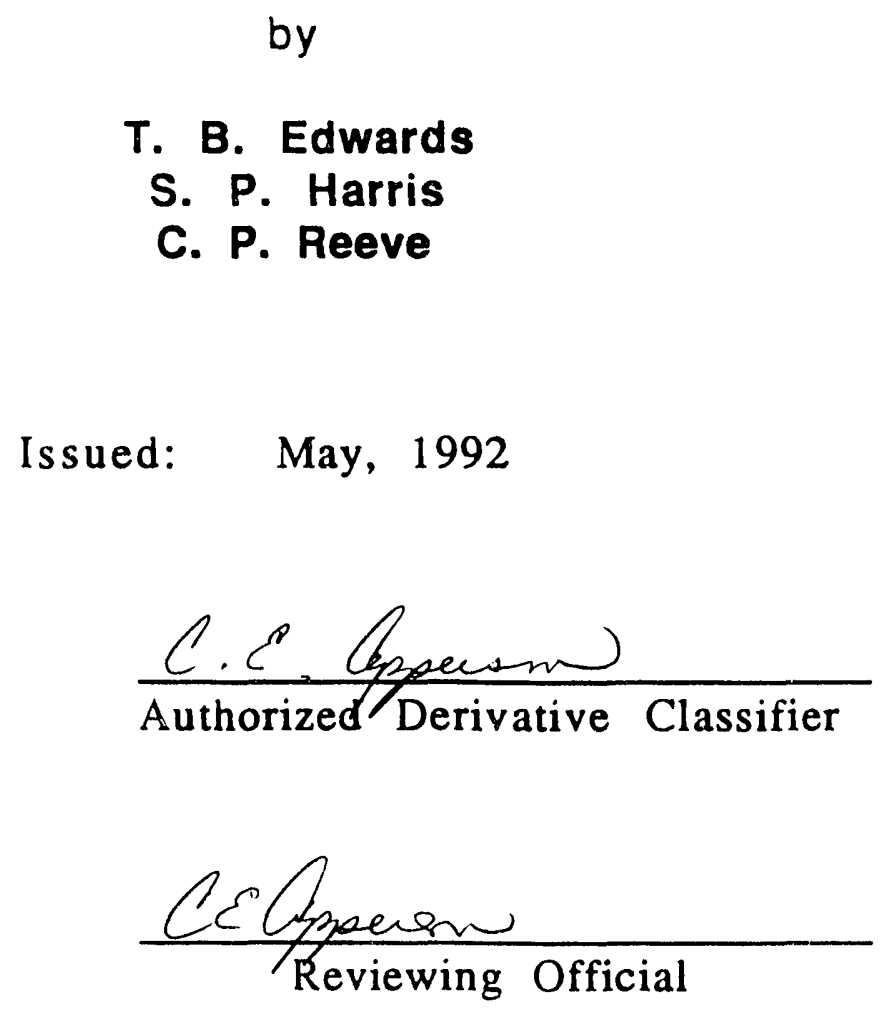

WSRC-TR-92-259

Task 92-053-1

SRTC

MATER

Savannah River Technology Center, Aiken, SC 29802

Westinghouse Savannah River Company

Prepared by the U. S. Department of Energy under Contract DE-AC09-8\$SR18035 
Document:

Title:

Task Number:
W SR C - TR - $92-259$

Statistical Analysis of Test Data for APM Rod Issue (U)

$92-053-1$

Approvals

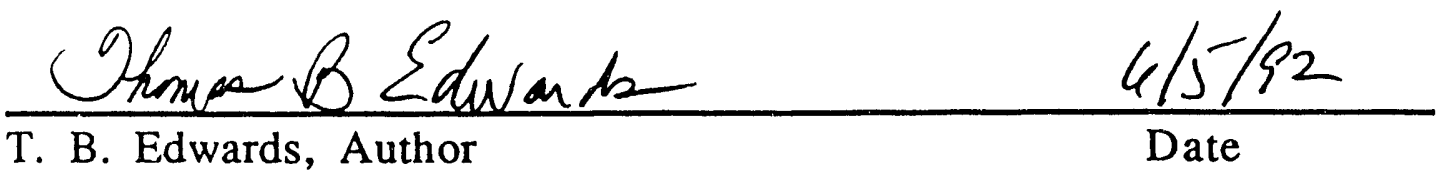

T. B. Edwards, Author

Date
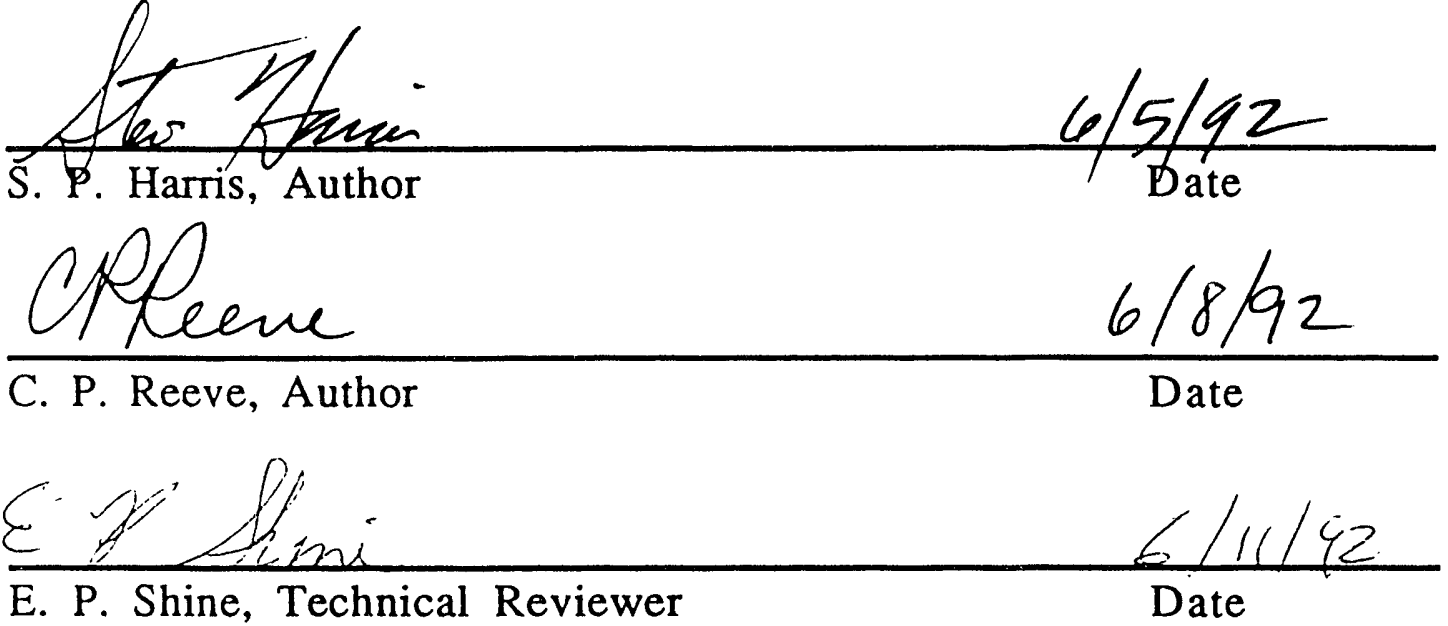

E. P. Shine, Technical Reviewer

Date
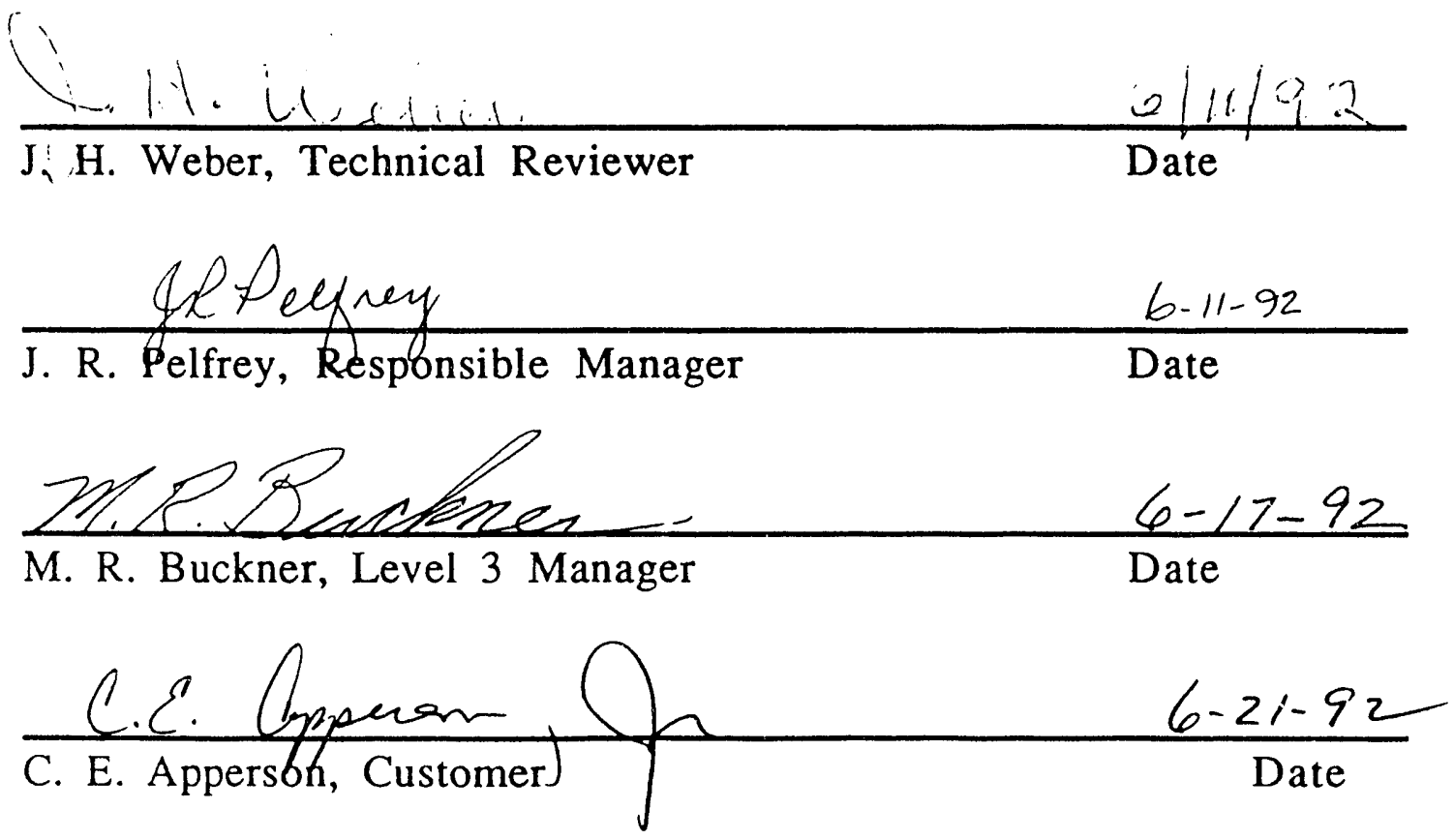


\section{Table of Contents}

Section

Page

1.0 Executive Summary

$2.0 \quad$ Introduction ............................................................

$3.0 \quad$ Test Data ................................................................ 2

4.0 Statistical Approach .................................................. 2

$5.0 \quad$ Inverse Prediction Problem .................................. 5

6.0 Total System Uncertainty ....................................... 7

7.0 Conclusions .................................................................. 8

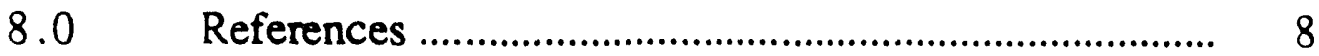

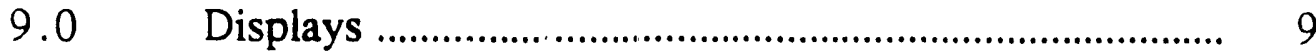

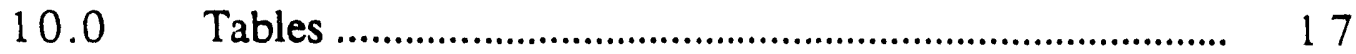

$11.0 \quad$ Appendices ............................................................ 54 


\subsection{Executive Summary}

The uncertainty associated with the use of the K-Reactor axial power monitors (APMs) to measure roof-top-ratios is investigated in this report. Internal heating test data acquired under both DC-flow conditions and $\mathrm{AC}$-flow conditions have been analyzed. These tests were conducted to simulate gamma heating at the lower power levels planned for reactor operation. The objective of this statistical analysis is to investigate the relationship between the observed and true roof-top-ratio (RTR) values and associated uncertainties at power levels within this lower operational range. Conditional on a given, known power level, a prediction interval for the true RTR value corresponding to a new, observed RTR is given. This is done for a range of power levels. Estimates of total system uncertainty are also determined by combining the analog-to-digital converter uncertainty with the results from the test data.

\subsection{Introduction}

A concern was raised regarding the axial power monitor (APM) rods as they are used to measure the roof-top-ratio (RTR) for the K-Reactor. RTR is a measure of relative power between the top and the bottom of the core. The objective is to investigate the relationship between the observed and true roof-top-ratio (RTR) values and associated uncertainties at power levels within the planned operational range for the reactor. The approach is based on a statistical analysis of test data acquired as part of the overall resolution of these APM concerns.

The APMs are self-calibrating gamma thermometers that were manufactured by the Delta-M Corporation. There are nine APM rods in the reactor. Each rod contains seven sensors along their axial dimension. The RTR value as observed by the reactor operator is the ratio of the response of Sensor \#2 to the response of Sensor \#6 as determined by the Control Computer.

Since the rods were initially designed and procured when operating levels for the SRS reactors were around $2400 \mathrm{MW}$, additional performance studies of the rods in the planned reactor operating range (720 MW) were initiated. Tests were conducted using internal heaters to simulate reactor gamma heating. The data from these tests were recorded using the APM data acquisition system (DAS). Additional data used for corroborating results were collected using the Control Computer.

A statistical analysis of these data, recorded over the lower power levels, forms the basis for the investigation of the RTR uncertainties. Prediction equations for observed RTR values are developed by applying the method of least squares regression to the test data. The uncertainties associated with these predictions are estimated. These results are then used to determine prediction intervals for the true RTR values corresponding to new, observed RTRs for power levels within the range of interest. 
The total system uncertainty is estimated by modifying the results from the statistical analysis of the test data to include the analog-to-digital converter (ADC) uncertainty.

\subsection{Test Data}

A number of tests were conducted to provide data for the analysis of RTR uncertainties. All of these tests involved internal heater experiments conducted for the nine APM rods in the K-Reactor. Data were collected under two different flow conditions. Initially, tests were conducted under flow conditions with all 6 DC pumps operating. Data from all nine APM rods were acquired using the APM DAS and some data for APM positions 5 through 9 were also acquired using the Control Computer. In addition, some tests were repeated. A second series of tests were conducted under flow conditions with all AC pumps operating. For these tests, data from all nine APM rods were acquired using the APM DAS.

This report provides the analysis for bcth the $D C$ and $A C$ flow test data acquired using the APM DAS as presented in appendices 1-5 for the DC tests and 6-10 for the AC tests. Only the power level ( $X$, measured in watts/gram), the sensor 2 response ( $Y 2$, given in $m V$ ), and the sensor 6 response ( $Y 6$, given in $m V$ ) are provided. The data from these appendices for $X$ greater than zero were used in this analysis. Test data collected with the Control Computer and some replicate data acquired with the APM DAS were used as corroborating information.

\subsection{Statistical Approach}

To estimate the uncertainty of the RTR values, the relationship between sensor response and power must be investigated. Displays $1-2$ provide charts of the responses of sensors 2 and 6 versus the watts/gram power level for each of the 9 APM rods under DC-flow conditions. Note that there is an anomaly in the behavior of APM 6 for power values above 1.2 watts/gram. Display 3 demonstrates a consistency in this behavior for APM 6 across the other. corroborating DC-flow test data. Displays 4-7 provide charts similar to displays 1-2 except they cover the AC-flow tests. Displays 4 and 5 cover the range 0 to 2 watts/gram and displays 6 and 7 cover the range 0 to .4 watts/gram. Note that there are no anomalies for APM 6 in the AC-flow data.

\section{Least Squares Regression}

The method of least squares regression was used to investigate the relationship between sensor response in $\mathrm{mV}$ to power level in watts/gram. Linear and quadratic models were explored. The results from these investigations as well as information from the manufacturer of the APM rods [1] and engineering considerations led to the selection of a quadratic prediction equation with no intercept term. Such a model was determined for each of the sensors ( 2 and 6 ) 
for each of the nine APM rods. The estimates of the coefficients of the quadratic equations along with their variances and covariances are provided in Appendix 11 for the DC tests and Appendix 12 for the AC tests.

These models were estimated using SAS-PC Release 6.04, a commercial software package from SAS Institute, Inc., Cary, NC. The code developed using this package is presented in A.ppendix 13.

The residuals from the quadratic fits for sensors 2 and 6 were investigated for each APM position. A positive correlation exists between the two sets of residuals for each rod. The approach taken here, which is to assume that these errors are uncorrelated, leads to more conservative (larger) estimates of the random error variances in the discussion below.

\section{Systematic Error Of Predicted RTR Value}

An observed RTR value for an APM rod is determined by the ratio of responses of sensor 2 to sensor 6 . Let the true quadratic response curves of the sensors be given by

$$
Y_{2}=A_{2} x+B_{2} x^{2}+\varepsilon_{2} \quad \text { and } \quad Y_{6}=A_{6} x+B_{6} x^{2}+\varepsilon_{6}
$$

where $x$ is the power level in watts/gram applied to that sensor, the A's and B's are unknown constants, and the $\varepsilon$ 's are uncorrelated, random variables which are assumed to have zero mean.

Let $x$ be the power applied to sensor 6 and $x T$ be the power applied to sensor 2 . Then the "true" RTR is given by

$$
x T / x=T .
$$

The A's and B's are estimated in the prediction equations determined by the method of least squares described above. If the estimates are represented by lowercase letters, then the predicted value of the observed RTR for given values of $x$ and $T$ is

$$
r(x, T)=\frac{a_{2} T+b_{2} \times T^{2}}{a_{6}+b_{6} \times}
$$

There is a systematic error associated with using these equations to predict RTR values. Each time a prediction equation is determined, the errors in the fitted equations are perpetuated as a systematic error in the predictions made with the equation.

An estimate of the variance of these systematic errors can be obtained by the method of error propagation as described in Appendix 14. Let

$$
\begin{array}{lll}
v_{a_{2}}=\operatorname{VARIANCE}\left(a_{2}\right), & v_{b_{2}}=\operatorname{VARIANCE}\left(b_{2}\right), \\
v_{a_{6}}=\operatorname{VARIANCE}\left(a_{6}\right), & v_{b_{6}}=\operatorname{VARIANCE}\left(b_{6}\right),
\end{array}
$$




$$
c_{a b_{2}}=\operatorname{COVARIANCE}\left(a_{2}, b_{2}\right), \quad c_{a b_{6}}=\operatorname{COVARIANCE}\left(a_{6}, b_{6}\right) .
$$

Estimates of these values are provided in appendices 11 and 12. The partial derivatives of interest are given by:

$$
\begin{aligned}
& \left(\partial r / \partial a_{2}\right)=T /\left(a_{6}+b_{6} x\right), \quad\left(\partial r / \partial b_{2}\right)=x T^{2} /\left(a_{6}+b_{6} x\right), \\
& \left(\partial r / \partial a_{6}\right)=-\left(a_{2} T+b_{2} x T^{2}\right) /\left(a_{6}+b_{6} x\right)^{2}, \\
& \left(\partial r / \partial b_{6}\right)=-x\left(a_{2} T+b_{2} x T^{2}\right) /\left(a_{6}+b_{6} x\right)^{2} .
\end{aligned}
$$

Then, the variance of the systematic error of the predicted, observed RTR value reduces to

Systematic Error Variance $=$

$$
\begin{aligned}
& \frac{T^{2} v_{a_{2}}+T^{4} x^{2} v_{b_{2}}+2 T^{3} \times c_{a b_{2}}}{\left(a_{6}+b_{6} x\right)^{2}} \\
& +\frac{\left(a_{2} T+b_{2} \times T^{2}\right)^{2}\left(v_{a_{6}}+x^{2} v_{b_{6}}+2 \times c_{a b_{6}}\right)}{\left(a_{6}+b_{6} x\right)^{4}}
\end{aligned}
$$

\section{Random Error Of Predicted RTR Value}

Next, the random error component of the uncertainty of the obsened RTR value is estimated. The random error of each sensor response can be estimated from the scatter around the quadratic prediction equation for each sensor. The responses of sensors 2 and 6 conditional on a known power ievel have standard deviations as computed from the determination of the quadratic prediction equations for each sensor. These values are provided in appendices 11 and 12. The estimated standard deviations of the random errors for sensors 2 and 6 are labelled as SIG_RND2 and SIG_RND6, respectively.

The observed RTR can be written as

$$
\begin{gathered}
r\left(\varepsilon_{\left.2, \varepsilon_{6}\right)}=r=\frac{A_{2} x T+B_{2}(x T)^{2}+\varepsilon_{2}}{A_{6} x+B_{6} x^{2}+\varepsilon_{6}}=\frac{\left(y_{2}+\varepsilon_{2}\right)}{\left(y_{6}+\varepsilon_{6}\right)}\right. \\
\text { where } \quad(\text { SIG_RND2 })^{2}=\text { Variance }\left(\varepsilon_{2}\right), \quad(\text { SIG_RND6 })^{2}=\text { Variance }\left(\varepsilon_{6}\right), \\
y_{2}=A_{2} x T+B_{2}(x T)^{2}, \quad y_{6}=A_{6} x+B_{6} x^{2} .
\end{gathered}
$$


The variance of the random error for $r$ is determined by error propagation as outlined in Appendix 14 using the following partial derivatives (note that $\varepsilon_{2}$ and $\varepsilon_{6}$ are uncorrelated):

$$
\begin{aligned}
& \left(\partial r / \partial \varepsilon_{2}\right)=1 /\left(A_{6} x+B_{6} x^{2}\right)=1 / y_{6}, \\
& \left(\partial r / \partial \varepsilon_{6}\right)=-\left(A_{2} x T+B_{2}(x T)^{2}\right) /\left(A_{6} x+B_{6} x^{2}\right)^{2}=-y_{2} /\left(y_{6}\right)^{2} .
\end{aligned}
$$

This approach leads to

$$
\text { Random Error Variance }=\left(1 / y_{6}\right)^{2}\left[(\text { SIG_RND2 })^{2}+\left(y_{2} / y_{6}\right)^{2}(\text { SIG_RND6 })^{2}\right] \text {. }
$$

\section{Total Uncertainty of Predicted RTR Value}

The total uncertainty (variance) of the observed RTR value is estimated by the sum of the systematic error (variance) and the random error (variance):

$$
\begin{gathered}
\text { Total Error Variance }= \\
\text { Systematic Error Variance + Random Error Variance. }
\end{gathered}
$$

For each APM rod, when the values of $x$ and $T$ are given, the prediction equations for the responses of sensors 2 and 6 allow the expected, observed RTR to be estimated. Tables 1-9 provide estimates of observed RTR values, denoted by P, for the APM rods for the DC tests. Tha uncertainty for these RTR values from (1) may be expressed as a percent relative standard deviation which is denoted in the tables as PCT_TO:

$$
\text { PCT_TO }=100 \frac{\sqrt{\text { Total Error Variance }}}{\mathrm{P}} .
$$

The results for the $A C$ tests are given in tables 10-18. The other columns presented as part of these tables will be discussed below.

\subsection{Inverse Prediction Problem}

The relationship between the observed RTR values and true RTR values, given the power level $x$, is investigated in this section. In operation, an RTR value, based on the responses of sensors 2 and 6 , is observed by the operator. Denote this value by $r_{0}$. The operator does not know the $x$ or $T$ values corresponding to this observed $r_{0}$. Given that $x=x_{0}$, an estimate of $T, T_{0}$, corresponding to $r_{0}$ can be determined by inverting the prediction equation for $r$. A prediction interval for $T$ can be determined based on the uncertainties for the new, observed RTR value, $r_{0}$, given $x_{0}$. The uncertainties (systernatic error and random error) can be combined in an additive approach instead of the 


\section{Statistical Analysis of Test Data for APM Rod Issue Page 6 of 87}

quadrature approach of equation (1) to provide a conservative, 95\% prediction interval for $r_{0},\left(r_{L}, r_{U}\right)$. This method is used in [4] and gives:

$$
\begin{array}{r}
r u=r_{0}+2[\text { Systematic Error Standard Deviation } \\
+ \text { Random Error Standard Deviation ] } \\
r_{L}=r_{0}-2[\text { Systematic Error Standard Deviation } \\
+ \text { Random Error Standard Deviation ]. }
\end{array}
$$

These prediction limits can be used to determine a prediction interval for the value of $T$, the true RTR, corresponding to $r_{0}$ given $x_{0}$. Consider the following graphic representation of the prediction curve and prediction intervals.

Relationship Between r (Observed RTR) and T (True RTR) Given $x_{0}$

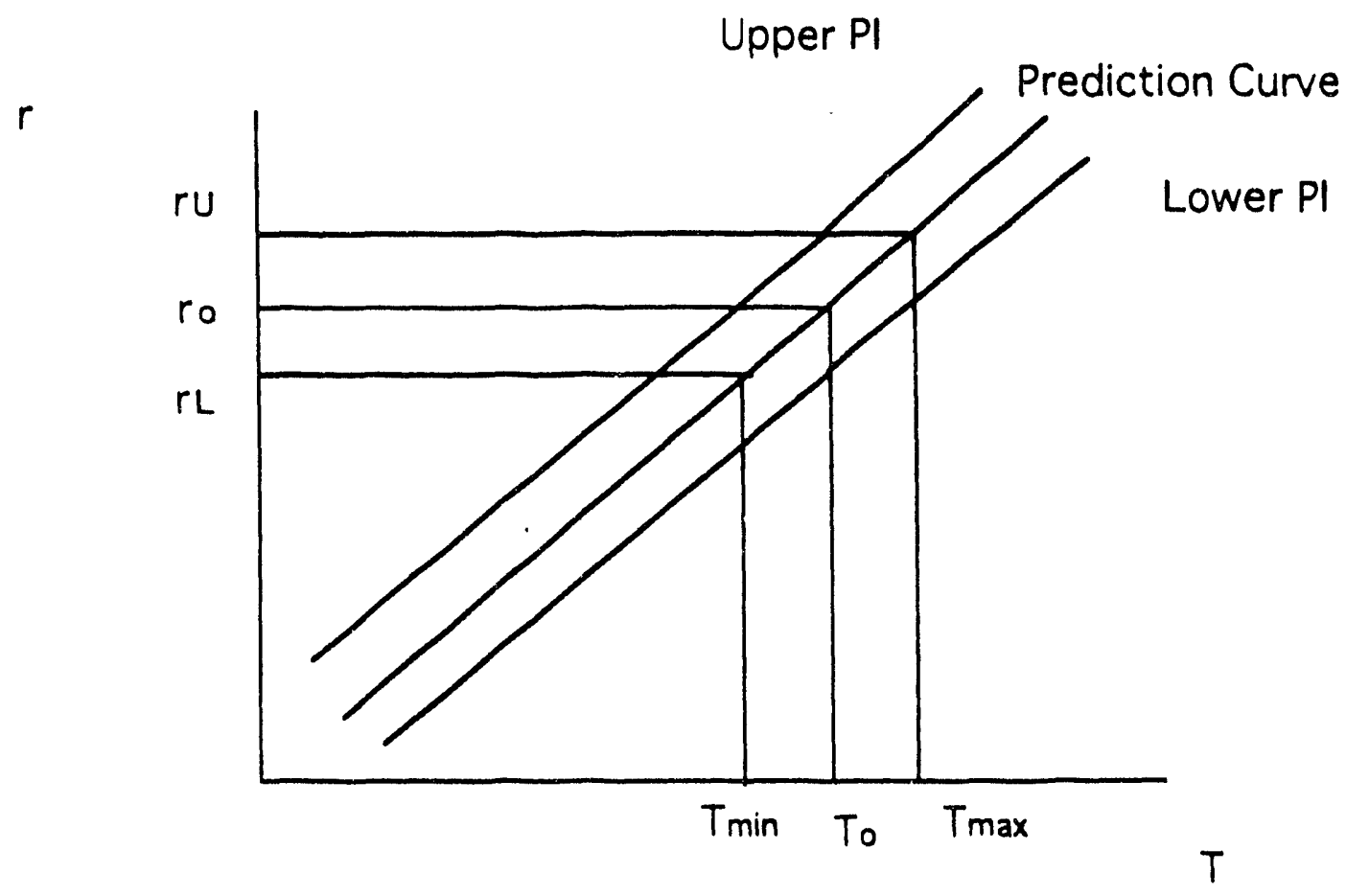

When the observed RTR value is within its bounds, $\left(r_{L}, r_{U}\right)$, on the vertical axis, then the corresponding true RTR value will be within the limits $\left(T_{\min }, T_{\max }\right)$ for a given value of the power level, $x_{0}$. The equations for $T_{\min }$ and $T_{\max }$ are given by:

$$
T_{\max }=T_{0}+\frac{\left(r U-r_{0}\right)}{r^{\prime}\left(x_{0}, T_{0}\right)}, \quad T_{\min }=T_{0}-\frac{\left(r_{0}-r_{L}\right)}{r^{\prime}\left(x_{0}, T_{0}\right)}
$$

where $r^{\prime}\left(x_{0}, T_{0}\right)$ represents the derivative with respect to $T$ of the prediction function evaluated at $x_{0}$ and $T_{0}$ : 


$$
r(x, T)=\frac{a_{2} T+b_{2} \times T^{2}}{a_{6}+b_{6} x} .
$$

Applying this approach to the test data, the $T_{\min }$ and $T_{\max }$ values have been computed. They are presented in tables $1-9$ for the DC tests and tables 10-18 for the AC tests. Recall that $P$ is used in these tables to represent the observed RTR value, $r_{0}$.

\subsection{Total System Uncertainty}

A system uncertainty analysis was performed by the Instrumentation Systems and Setpoints Group following the methodology used in the commercial nuclear industry for investigating potential sources of uncertainties. The details of this analysis are presented in reference [2]. In summary, the test data and the statistical methodology developed here were evaluated and considered to be an adequate representation of the sensor uncertainties excluding the analog-todigital converter (ADC). Each time a sensor is read by the Control Computer, the ADC channel is used. The ADC channel uncertainty is applied independently to each sensor. To propagate this error to the RTR value, the following model can be used:

$$
r=\frac{A_{2} x T+B_{2}(x T)^{2}+\varepsilon_{2}+\eta_{2}}{A_{6} x+B_{6} x^{2}+\varepsilon_{6}+\eta_{6}}=\frac{\left(y_{2}+\varepsilon_{2}+\eta_{2}\right)}{\left(y_{6}+\varepsilon_{6}+\eta_{6}\right)}
$$

where $\varepsilon_{2}$ represents the random error for sensor 2, $\eta_{2}$ represents the ADC error for sensor 2 , $\varepsilon_{6}$ represents the random error for sensor 6 , and $\eta_{6}$ represents the ADC error for sensor 6 .

From [2], the $\eta$ 's are assumed to be independent, random variables with zero means. For sensor response within $\pm 10 \mathrm{mV}$, the maximum $A D C$ error contribution is $10 \mu \mathrm{V}$ (assume that this is a 3-sigma limit). Thus, the standard deviation of the ADC errors can be estimated as

$$
(10 \mu \mathrm{V}) / 3=3.33 \mu \mathrm{V}=.0034 \mathrm{mV}
$$

Once again the method of Appendix 14 can be used to propagate the error. The partial derivatives involved are as follows (note that $\varepsilon_{2}, \varepsilon_{6}, \eta_{2}$ and $\eta_{6}$ are pairwise uncorrelated):

$\left(\partial \mathrm{r} / \partial \varepsilon_{2}\right)=1 /\left(A_{6} x+B_{6} x^{2}\right)=1 / y_{6}, \quad\left(\partial r / \partial \eta_{2}\right)=1 /\left(A_{6} x+B_{6} x^{2}\right)=1 / y_{6}$, $\left(\partial r / \partial \varepsilon_{6}\right)=\cdot\left(A_{2} x T+B_{2}(x T)^{2}\right) /\left(A_{6} x+B_{6} x^{2}\right)^{2}=-y_{2} /\left(y_{6}\right)^{2}$, 
$\left(\partial r / \partial \eta_{6}\right)=-\left(A_{2} x T+B_{2}(x T)^{2}\right) /\left(A_{6} x+B_{6} x^{2}\right)^{2}=-y_{2} /\left(y_{6}\right)^{2}$

The technique of error propagation applied to this model leads to the following estimate for the variance of the total system random error

Random Error Variance $\mathrm{N}=$

$$
\left(1 / y_{6}\right)^{2}\left[\left(S I G \_R N D 2\right)^{2}+(.0034)^{2}+\left(y_{2} / y_{6}\right)^{2}\left[\left(S I G \_R N D 6\right)^{2}+(.0034)^{2}\right]\right] \text {. }
$$

There is no change in the systematic error due to the ADC since the converter was not a component of the APM DAS used to record the test data. Thus, the total system error variance is

$$
\text { Total Error Variance } \mathrm{N}=
$$

Systematic Error Variance + Random Error VarianceN.

New values for the percent relative standard deviation as given by (2) were computed using (5). New values for $T_{\min }$ and $T_{\max }$ were computed as in (4) using additive method for combining systematic and random errors as in (3). These values are included in tables 1.9 for the DC tests and tables 10-18 for the $A C$ tests. They carry the "_N" suffix to distinguish them from the previous estimates.

\subsection{Conclusions}

For values of the power level, the $x$ 's, between 0.05 and 0.4 watts/gram, tables 1.18 estimate the relationship between predicted, observed RTR values, the P's, and "true" RTR values, the T's, based on the test data. Uncertainties associated with this relationship are also determined including a prediction interval for $T$ based on a new, observed $P$ at a given value of $x$.

\subsection{References}

[1] Bayne, C. K., et al., "Operational Prediction Equations for APM Gamma Thermometer Rods: Volume I. Inverse Calibration Equations", Delta-M Corporation, March, 1990.

[2] Calc-Note: SRTC-ISS-92-9001, "Instrumentation/Setpoint Uncertainty Evaluation of the Axial Power Monitor," Task Number: SRL-RSC-91-8004.

[3] Jaech, John L. Statistical Methods in Nuclear Material Control, TID-26298, Technical Information Center, Office of Information Service, U.S. Atomic Energy Commission, December, 1973.

[4] ANSI N15.19-1989. "American National Standard for Nuclear Materials Control - Volume Calibration Techniques," American National Standards Institute, New York, 1989. 
WSRC-TR-92-259

Task 92-053-1

May 29, 1992

Revision 0

Statistical Analysis of Test Data for APM Rod Issue Page 9 of 87

9.0 Displays

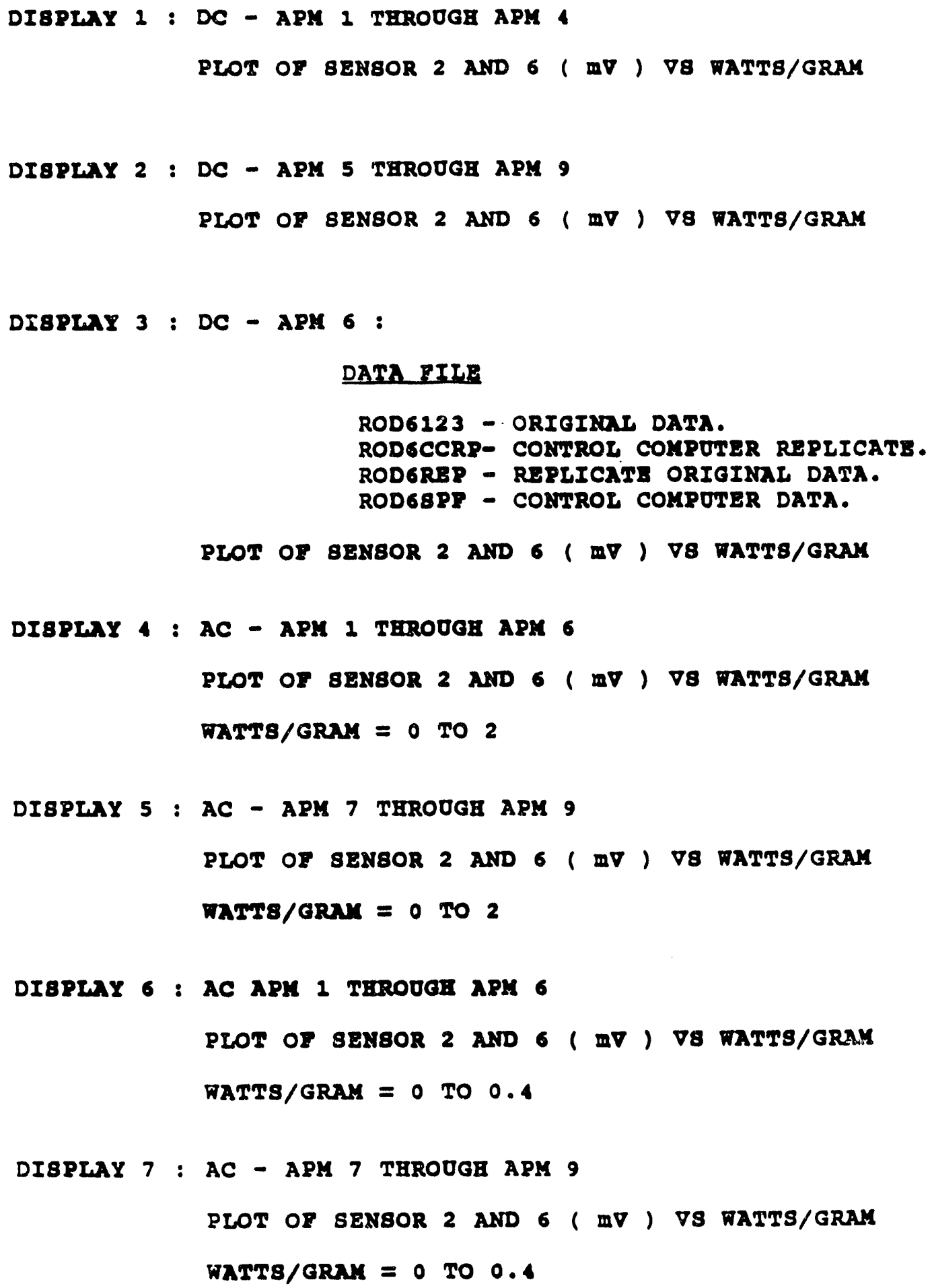




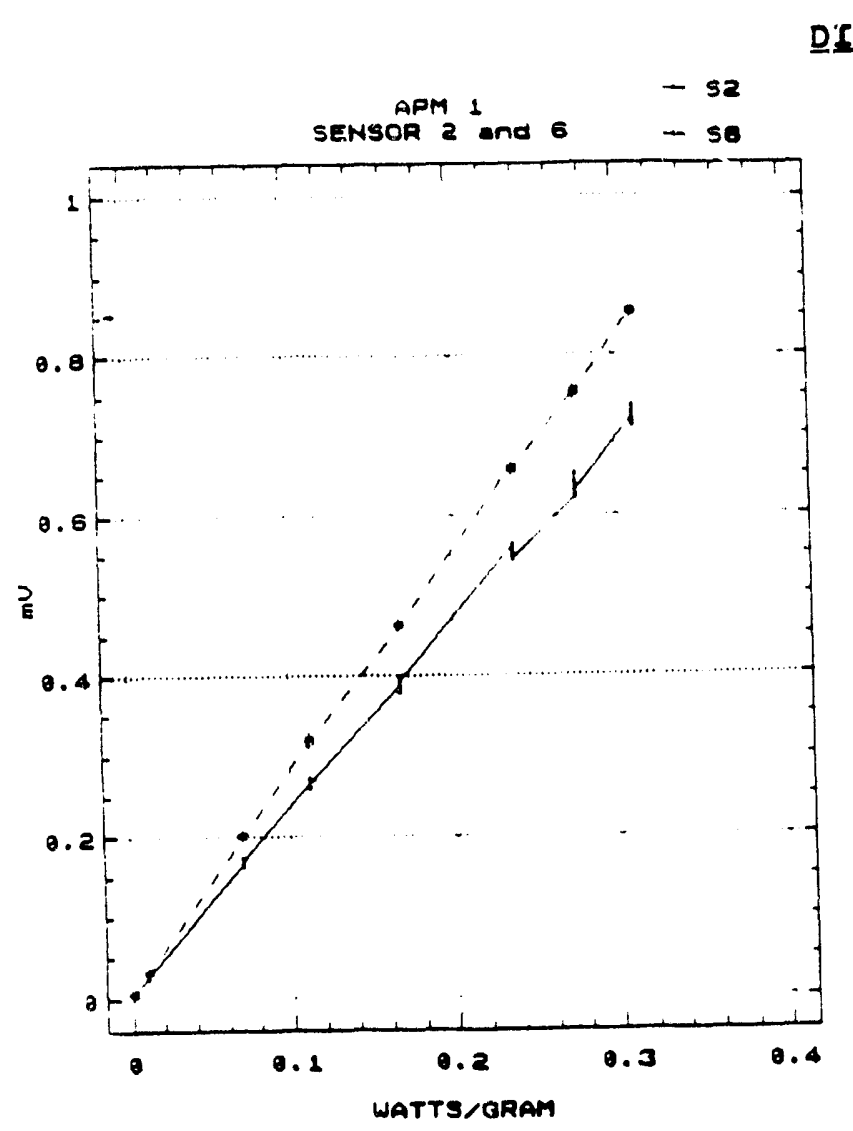

\section{Desprar 2}
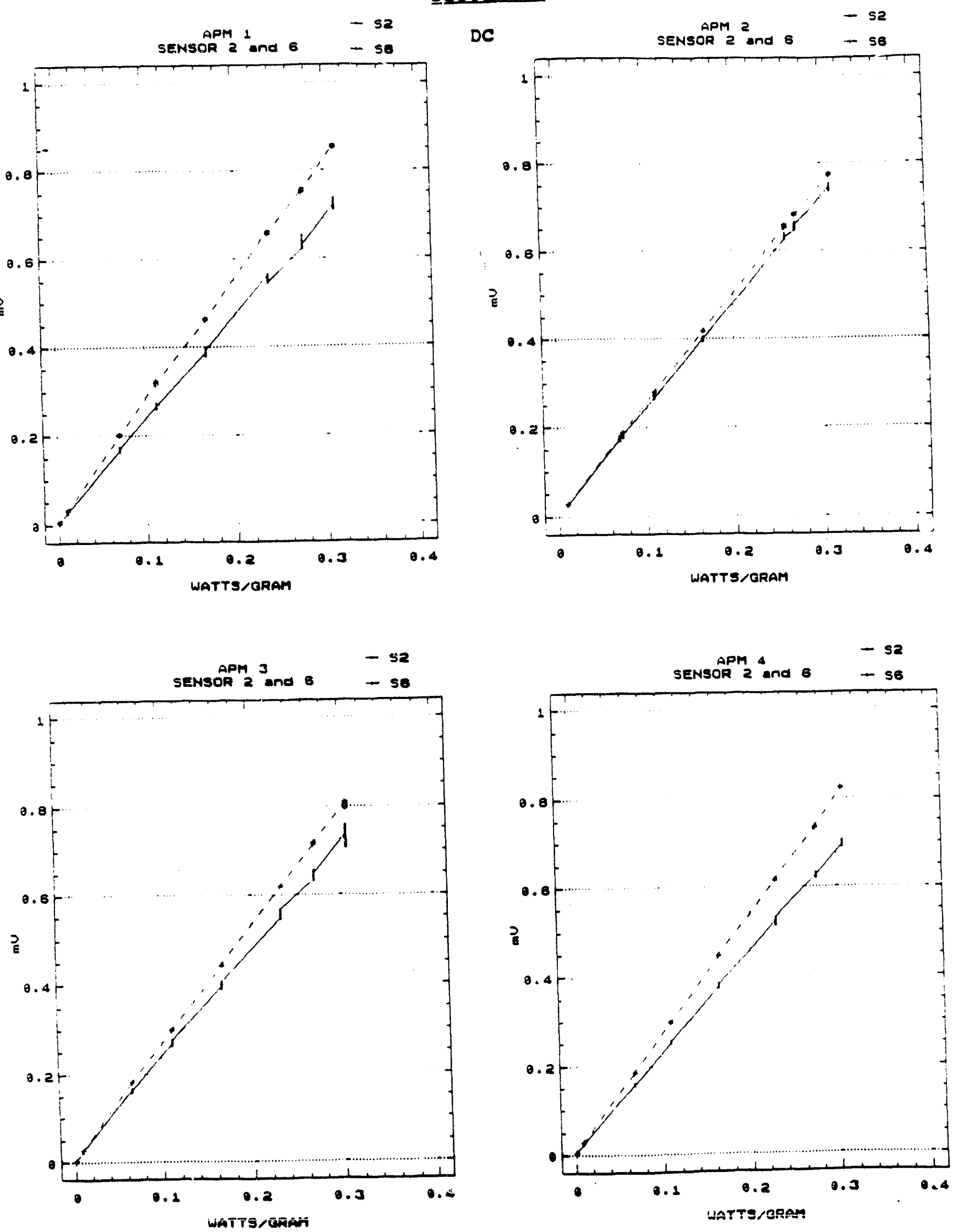
WSRC-TR-92-259

May 29, 1992

Task 92-053-1

Revision 0

Statistical Analysis of Test Data for APM Rod Issue Page 11 of 87
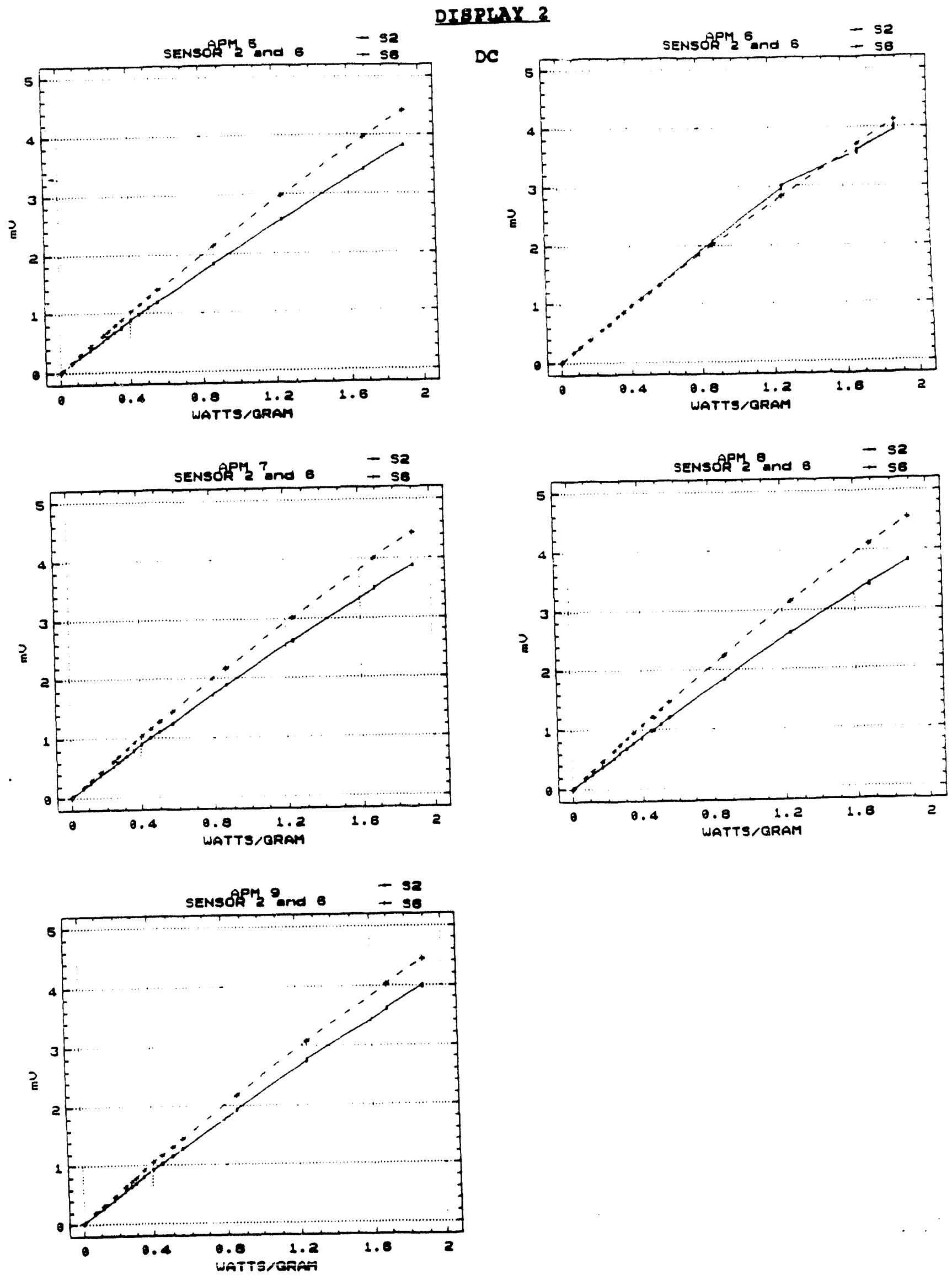


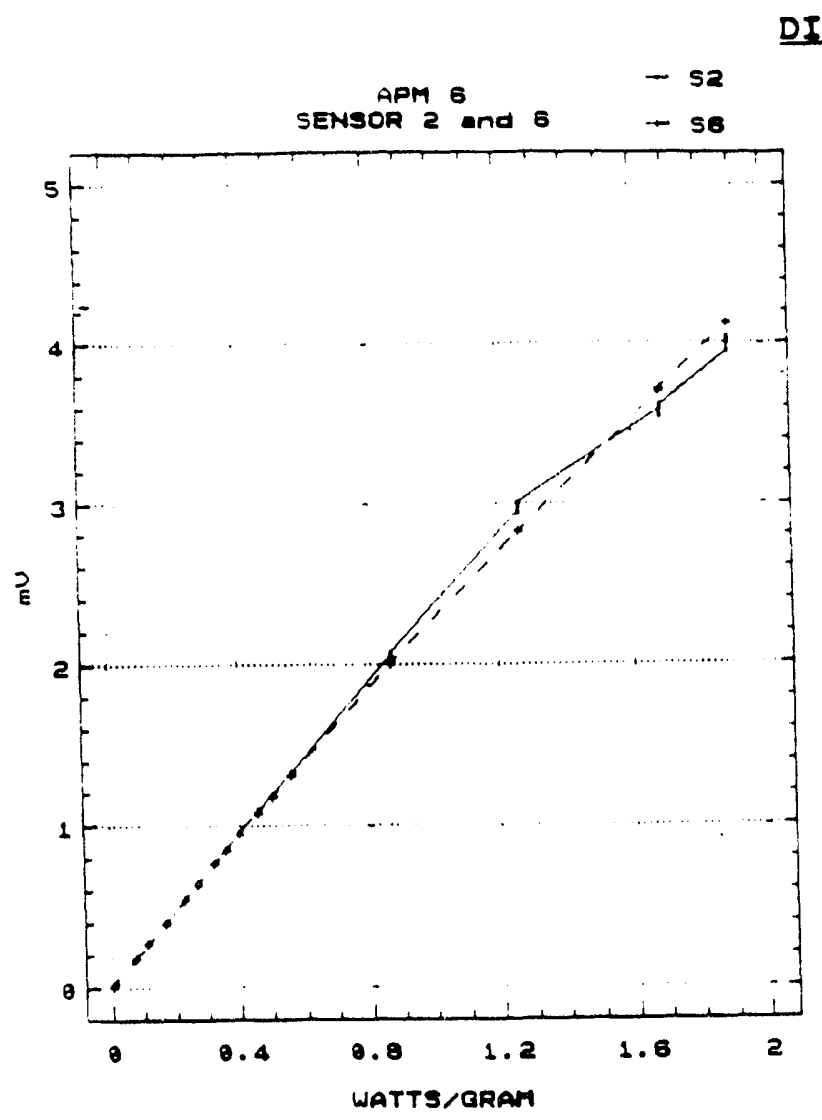

( R008123)

IBPLAY 3
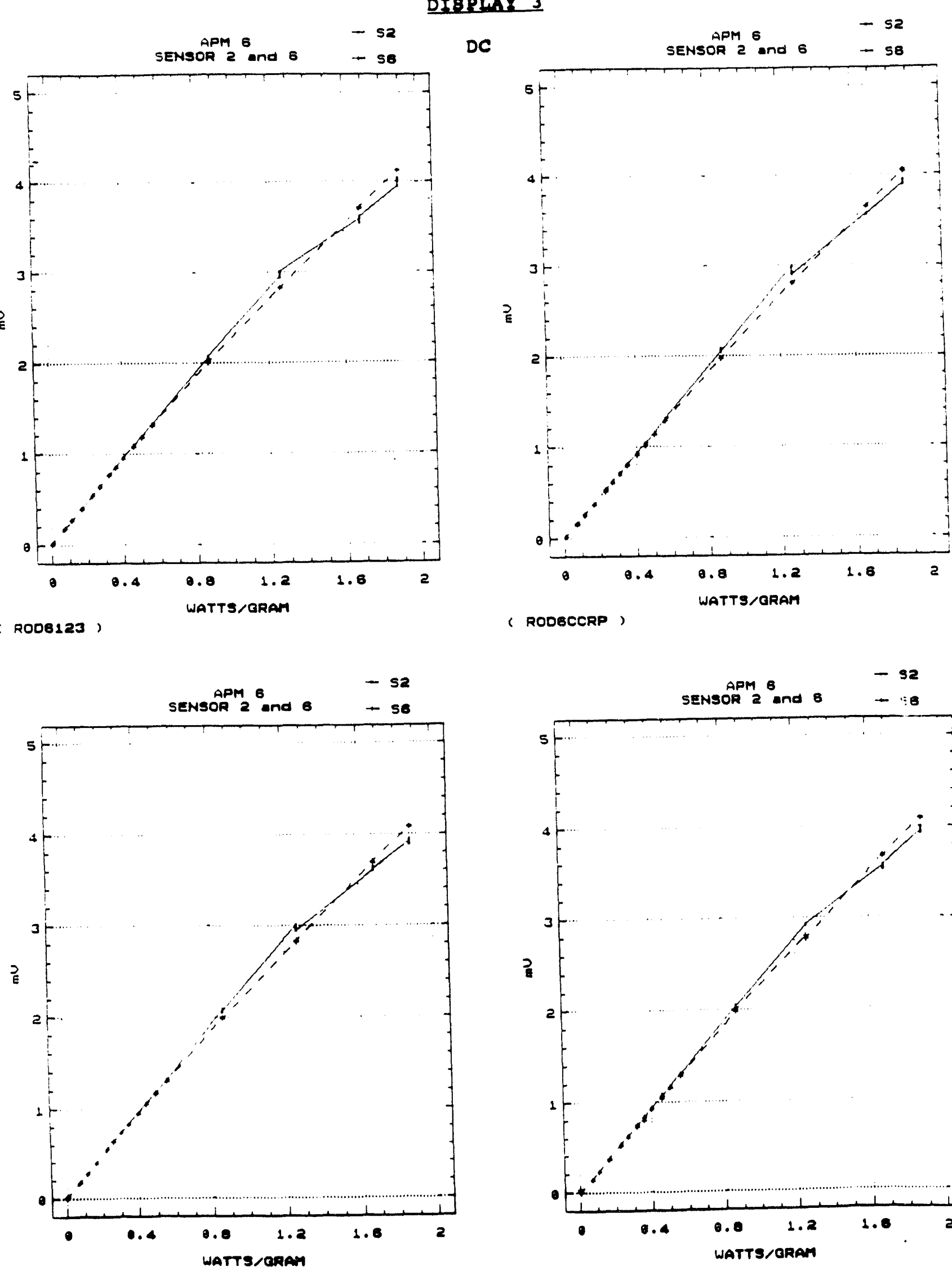

( RODERTR)

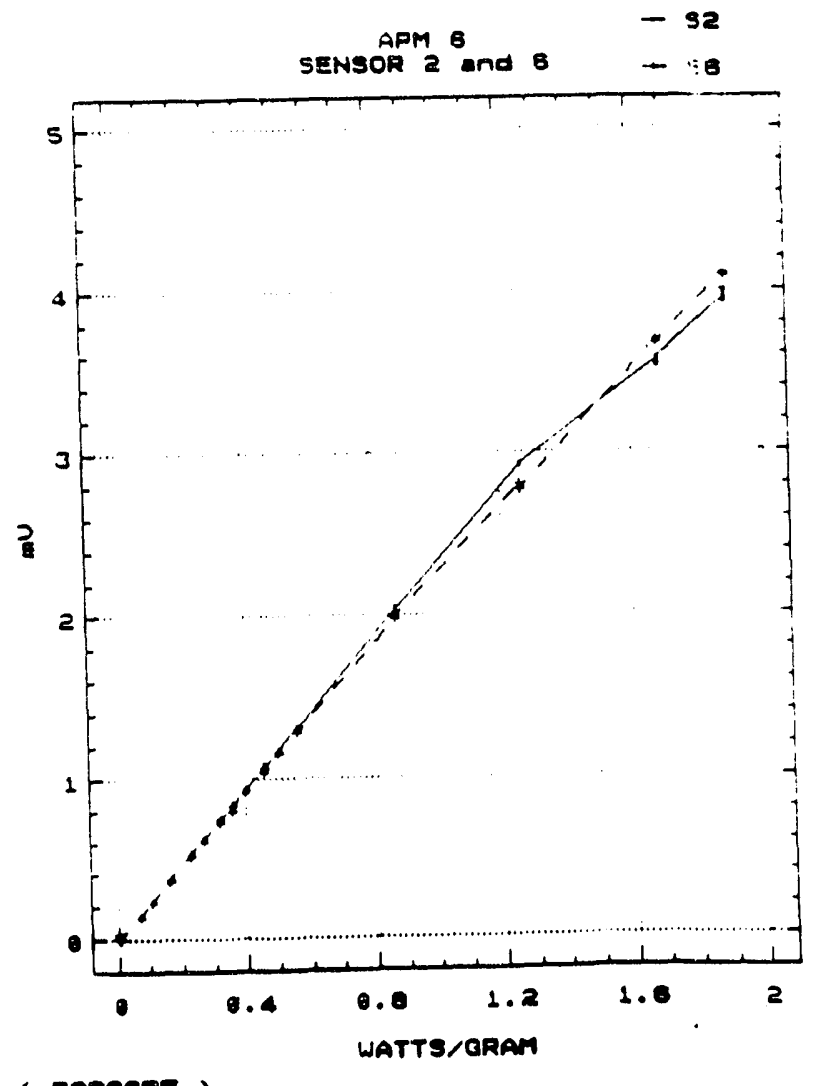




\section{DIBPTAY 4}

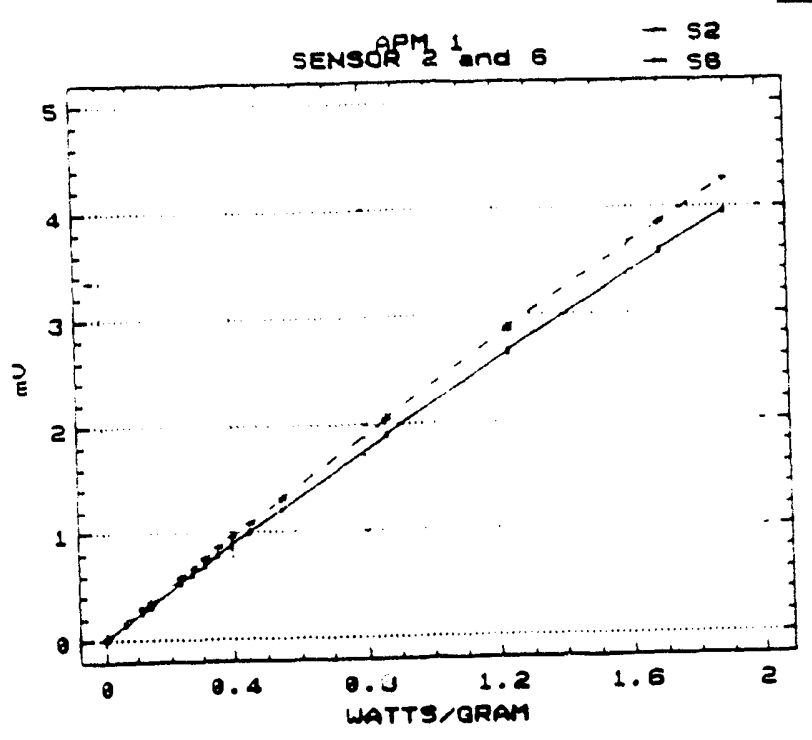

AC

SENSORR ${ }^{2}$ and 6

-52
-58
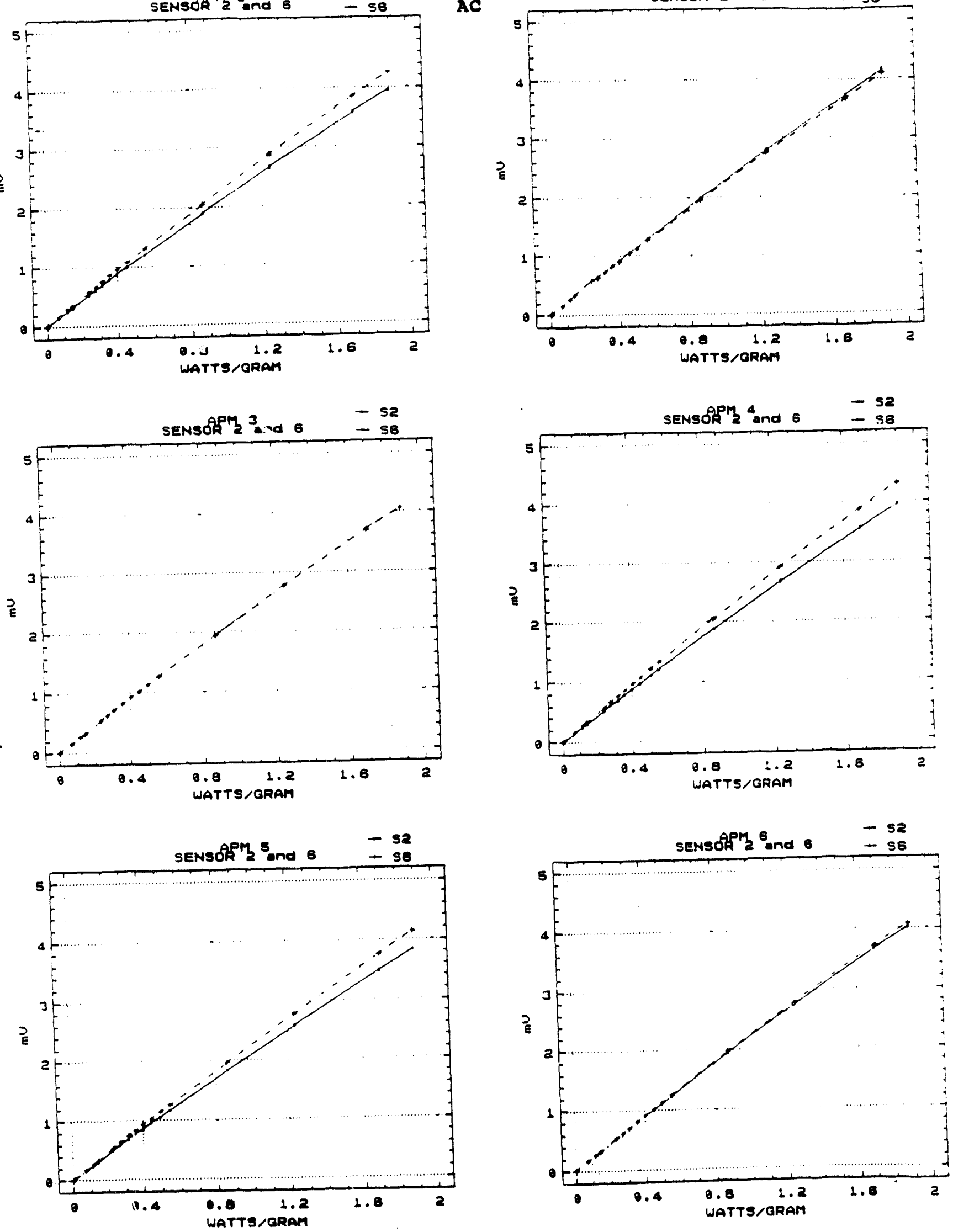

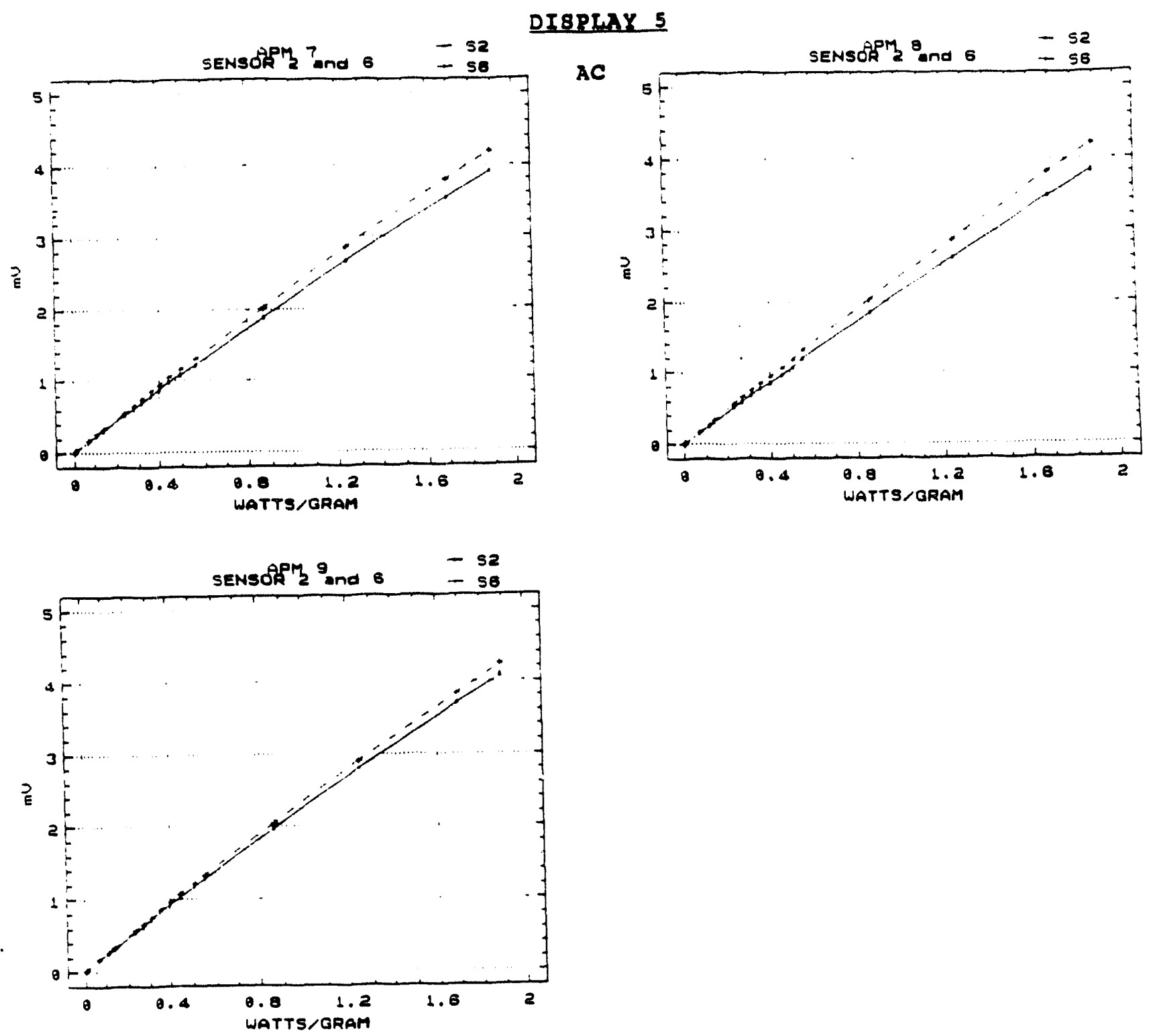


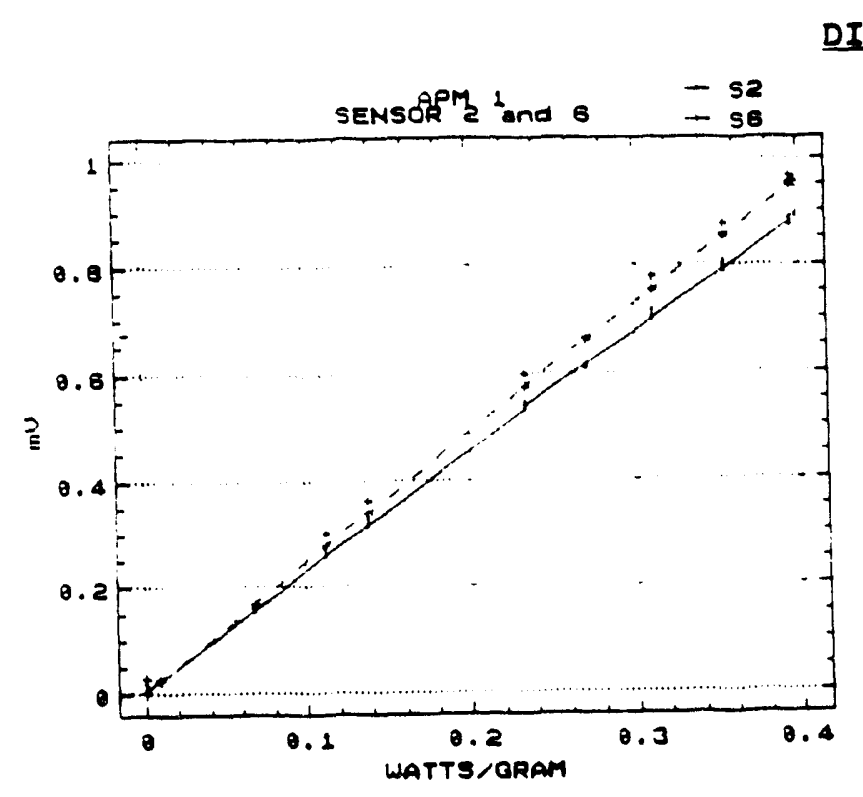

\section{DIBPHAY 6}
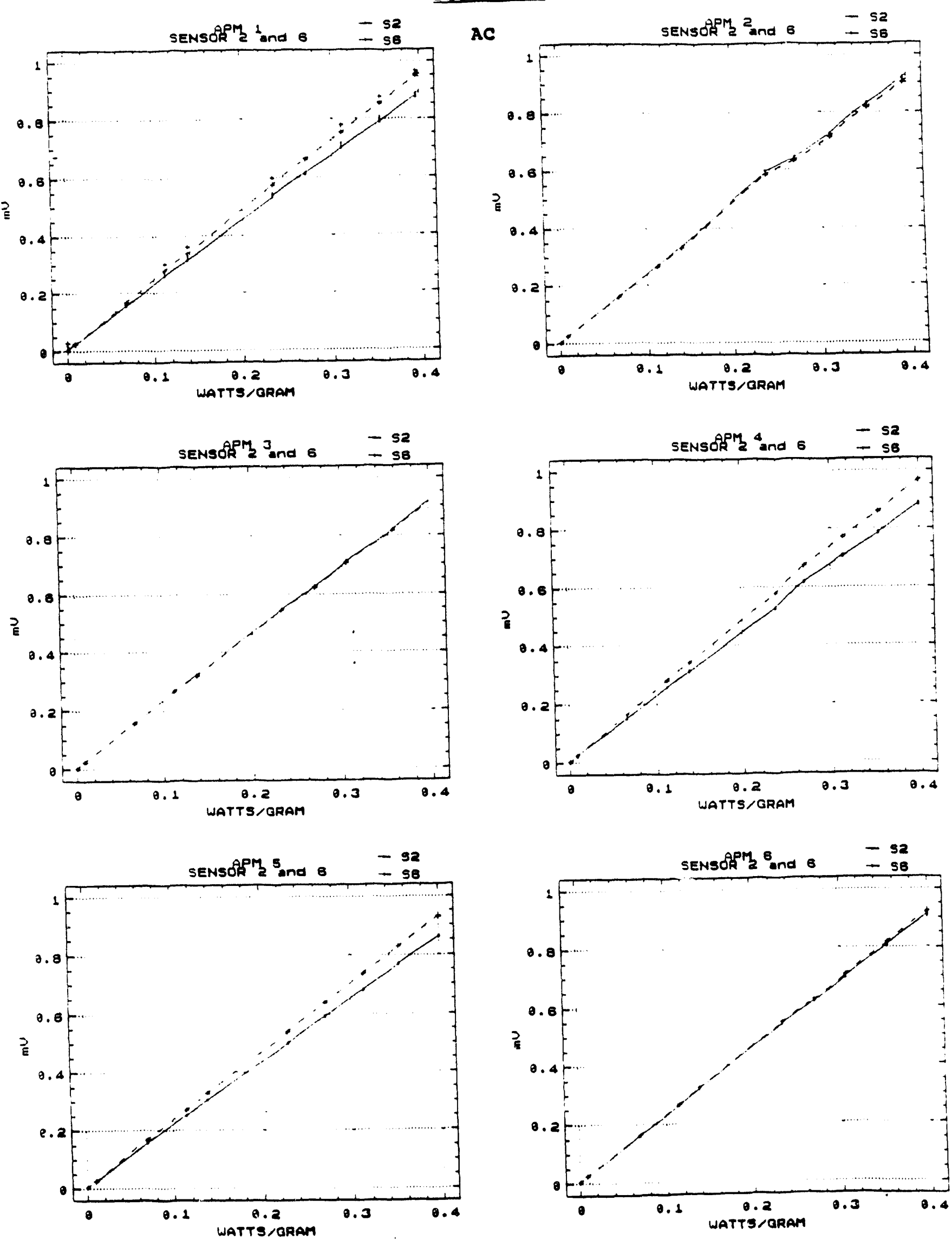


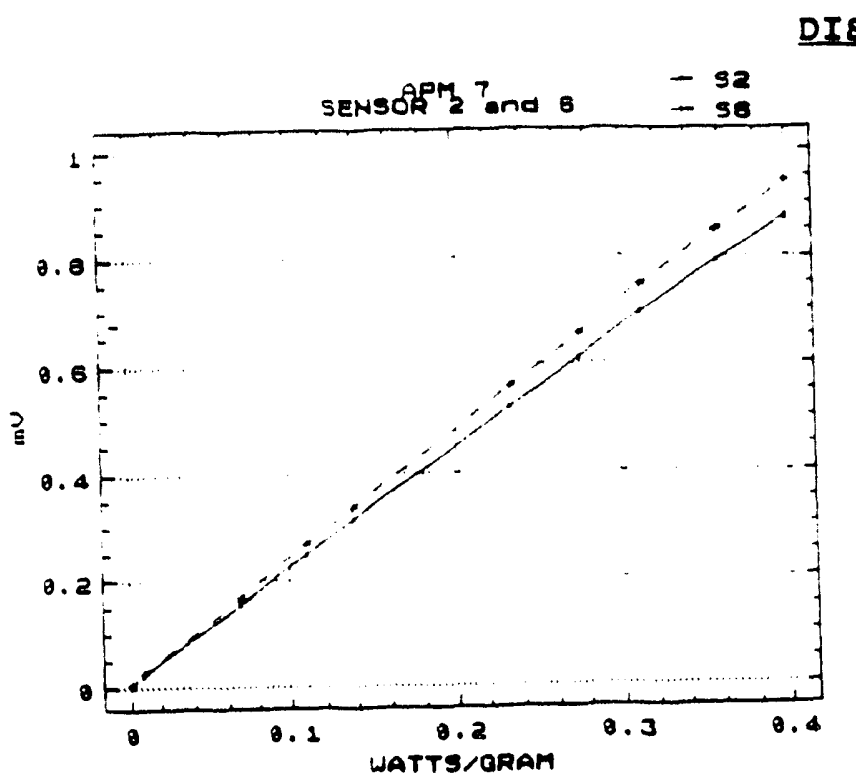

\section{DIBPLAY 7}
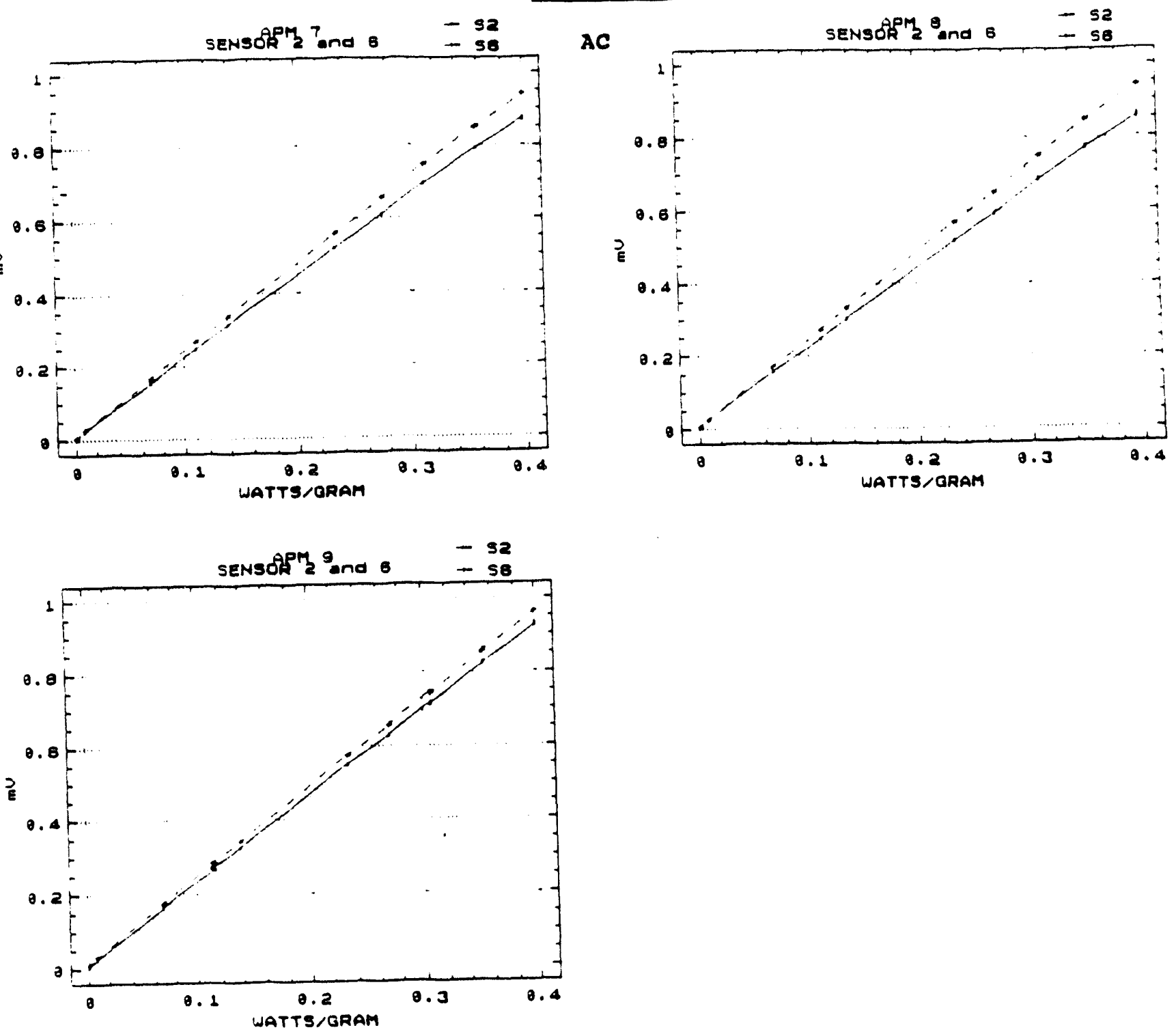
WSRC-TR-92-259

May 29, 1992

Task 92-053-1

Statistical Analysis of Test Data for APM Rod Issue Page 17 of 87

10.0 Tables

\begin{tabular}{|c|c|c|c|c|c|c|c|c|}
\hline TABLE & 1: & DC & - APY & 1 & TABLE & 10: & AC & - APM \\
\hline TABLE & $2:$ & DC & - APY & 2 & TABLB & 11: & AC & - APM \\
\hline ABLB & 3: & DC & - APM & 3 & TABLE & $12:$ & AC & - APM \\
\hline TABLE & 4: & DC & - APY & 4 & TABLB & 13: & AC & - APM \\
\hline TABLE & $5:$ & DC & - APM & 5 & TABLE & 14: & AC & - APY \\
\hline TABLB & $6:$ & DC & - APM & 6 & TABLE & 15: & AC & - ARM \\
\hline TABLE & $7:$ & DC & - APX & 7 & TABLE & $16:$ & AC & - APX \\
\hline TABLE & $8:$ & DC & - APY & 8 & TABLE & 17: & AC & - APM \\
\hline ABSE: & $9:$ & DC & $-\mathbf{A P}$ & $g$ & TABLE & 188 & AC & - APY \\
\hline
\end{tabular}

$$
\begin{aligned}
& X \text { = SENSOR } 6 \text { WATTS PER GRAY. } \\
& \text { T = UNOBgERVED ACTUAL ROOF-TOP RATIO. } \\
& \text { T_MIN = LOWBR } 95 \% \text { CONPIDENCB BOUND ON } \mathrm{T} \text {. } \\
& \text { T_MIN_N }=\underset{\text { IOWBR } 95 \% \text { CONTIDENCE BOUND ON } T}{T} \\
& \text { T_MAX = OPPER } 95 \% \text { CONPIDENCE BOUND ON } T \text {. } \\
& \text { T_MAX_N }=\text { OPPER 95* CONFIDENCE BOUND ON } T \\
& \text { P = EXPECTED ROOP-TOP RATIO. } \\
& \text { PCT_TO = PERCENT 8TANDARD DEVIATION OF } P \text {. } \\
& \text { PCT_TO_N= PERCENY 8TANDARD DEVIATIOA OF P }
\end{aligned}
$$


WSRC-TR-92-259

Task 92-053-1

May 29, 1992

Statistical Analysis of Test Data for APM Rod Issue

Revision 0

Page 18 of 87

TABLB 1

$(D C-A P M 1)$

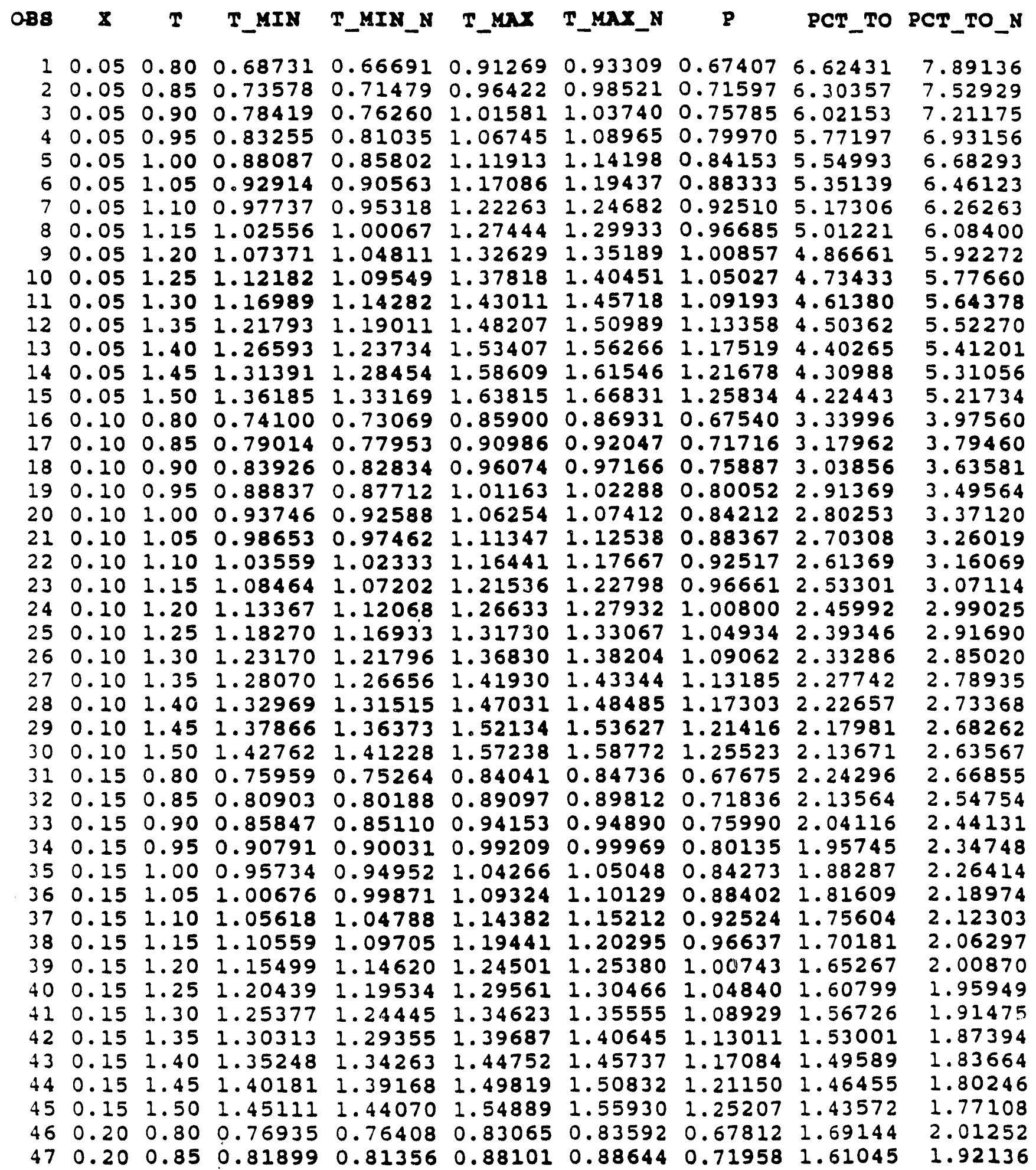


WSRC-TR-92-259

Task 92-053-1

May 29, 1992

Statistical Analysis of Test Data for APM Rod Issue Page 19 of 87

TABIE 1

( DC - APM I )

( CONTINOED )

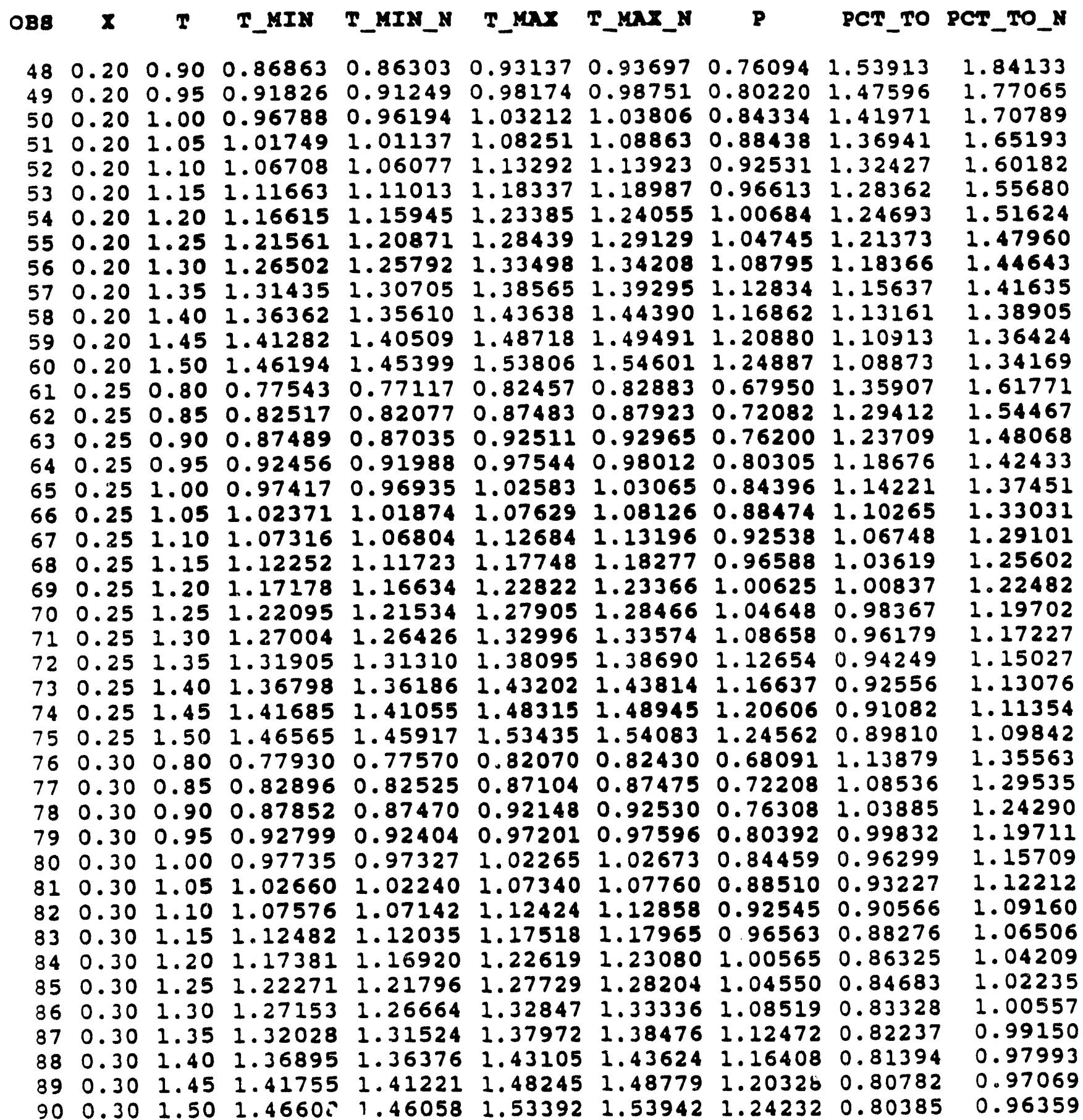




\section{TABLE 2}

$(D C-A P M 2)$

\begin{tabular}{|c|c|c|c|c|c|c|c|c|c|}
\hline 88 & $x$ & $\mathbf{T}$ & SIN & T_MIX_N & T_mx & r_max_y & E & PCT_TO & PCT_TO_M \\
\hline 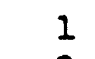 & & & & & & & & & \\
\hline & & & & & & & & & \\
\hline 3 & & & & 0052 & & 0.99948 & 0.86193 & & \\
\hline 4 & & & & & & 63 & & & \\
\hline 5 & & & & & & & & & \\
\hline 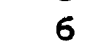 & & 1.05 & & & 21 & & 1.00487 & & \\
\hline & & 1.10 & & & .17 & & 1.05247 & & \\
\hline 8 & & 1.15 & 1.078 & 1.0 & 1.22 & 1.26 & 1.10004 & & \\
\hline 9 & & 1.20 & 1.12 & 1.0 & & & & & \\
\hline & & 1.25 & 1.17 & & & & & & \\
\hline & & 1.30 & 1.22 & 1.18 & & & & & \\
\hline & & 1.35 & 1.27 & 1.22 & 1.42 & & 1.2 & & \\
\hline & & 1.40 & 1.32 & 1.2 & 1.47 & & 1.3 & & \\
\hline & & 1.4 & 1.3 & 1.3 & 1.5 & & 1.3 & & 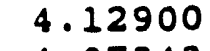 \\
\hline & .0 & .00 & 5 & 1.3 & & & & & \\
\hline & & & & & & & & & 391 \\
\hline & & 0.85 & & & & & & & \\
\hline & 0.3 & 0.90 & 0.8 & & & 0.5 & & & \\
\hline & 0.10 & 0.95 & 0. & $0 . \varepsilon$ & 0 & 1.0 & & & 935 \\
\hline & 0.10 & 1.00 & & & & & & & \\
\hline & 0.10 & 1.05 & & 0.9 & & & & & \\
\hline & & 1.10 & $1 . c$ & & 1.1 & & 1.0 & & \\
\hline 25 & 0.18 & 1.15 & 1.11 & 1.0 & 1.1 & 1.2 & 1.1 & & \\
\hline & 0.1 & 1.20 & 1. & 1.2 & & 1.2 & & & 590 \\
\hline & 0.10 & 1.25 & 1.21 & 1.1 & 1.28 & 1.3 & 1.1 & & 123 \\
\hline & 0.10 & 1.30 & 1.26 & 1.2 & 1.3 & & & & \\
\hline 2 & & 1.35 & 1.2 & 1.2 & 1.3 & & 1.2 & & \\
\hline 28 & 0.10 & 1.80 & 1.3 & 1.3 & 1.4 & & 1.3 & & \\
\hline 29 & & & & 1.3 & & & 1.3 & & 27 \\
\hline 30 & & & 1.45 & 1.4 & 1.5 & & 1.4 & & 238 \\
\hline 31 & & & & 0.7 & & & & & \\
\hline & & 0.85 & 0.8 & & & & & & \\
\hline $3:$ & & 0.90 & 0.87 & & & & 0.8 & & \\
\hline & & & & & & & & & \\
\hline 35 & & 1.00 & 0.97 & 0.9 & 1.02 & & 0.9 & 1. & 740 \\
\hline 36 & & & 1.02 & 1.0 & & & 1.0 & & \\
\hline 3 & & 1.10 & 1.07 & & & & & & \\
\hline & & & 1.12 & & & & & & \\
\hline 3 & & 1.20 & 1.17 & 1.3 & 1.22 & & 1.1 & & 000 \\
\hline 40 & & 1.25 & 1.22 & 1.2 & 1.27 & & 1.1 & & 013 \\
\hline 41 & & 1.30 & 1.27 & 1.2 & 1.32 & & 1.2 & & 311 \\
\hline & & 1.35 & 1.32 & 1.3 & 1.37 & & 1.28973 & & \\
\hline & & 1.40 & 1.37 & & 1.42 & & & & 627 \\
\hline 4 & & 1.45 & $1.42 \%$ & & 1.47 & & 1.3 & & \\
\hline 45 & 0.15 & 1.50 & 1.471 & 1.45522 & 1.5 & & 1.4 & 0.84 & 1730 \\
\hline 46 & 0 . & & 0.782 & 0.7747 & 0.81 & & 0.7 & & \\
\hline & & & 3265 & 2423 & 0.86735 & 7577 & 0.81984 & 0.91310 & L. 3984 \\
\hline
\end{tabular}


WSRC.TR-92-259

Task 92-053-1

May 29, 1992

Revision 0

Statlstical Analysis of Test Data for APM Rod Issue Page 21 of 87

TABLB 2

$(D C-A P M 2)$

( CONTINOED )

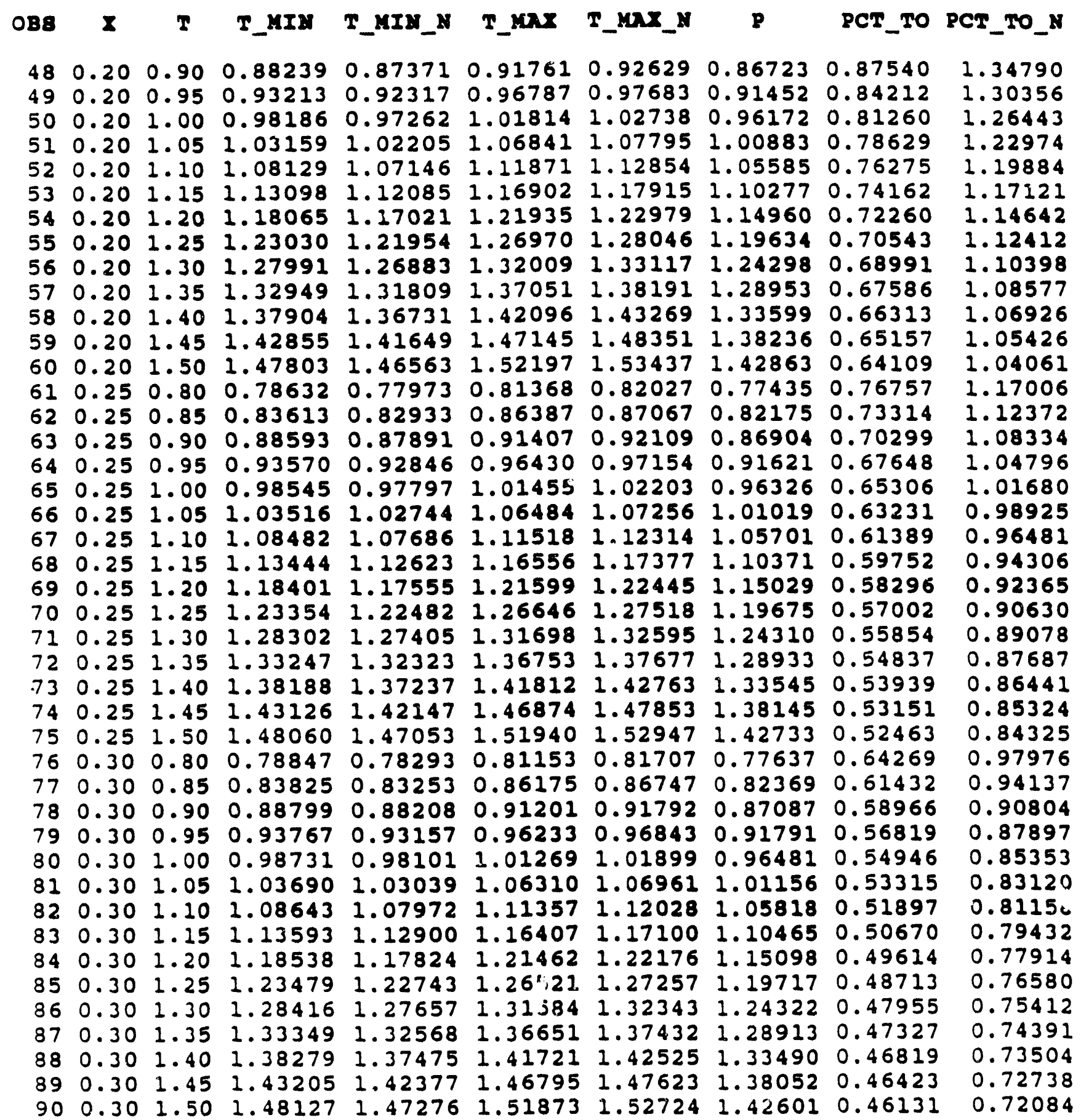




\title{
TABLE 3
}

\author{
( DC - APM 3 )
}

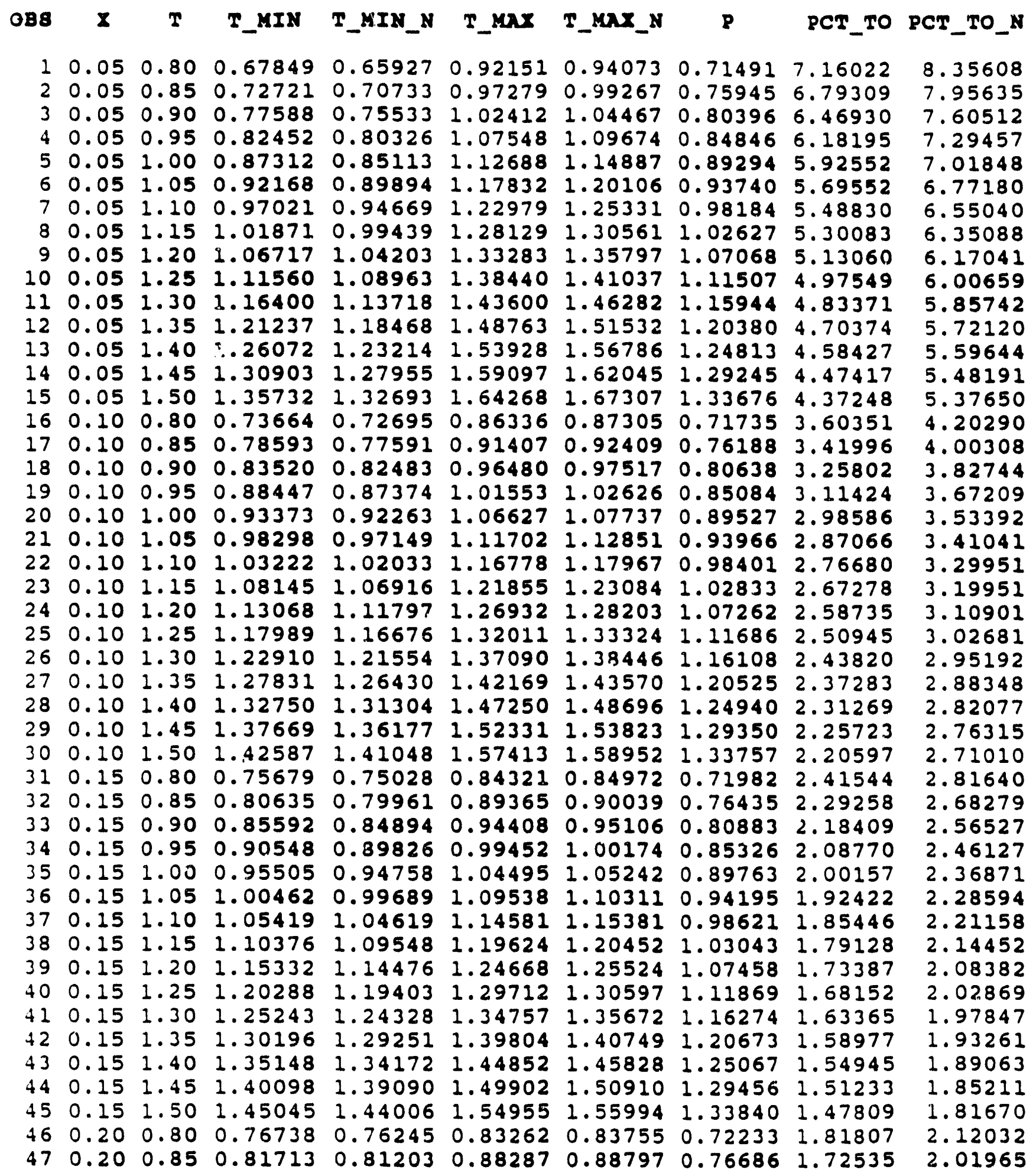


WSRC-TR-92-259

Task 92-053-1

May 29, 1992

Statistical Analysis of Test Data for APM Rod Issue

Revision 0

Page 23 of 87

TABLE 3

( DC - APY 3 )

( CONTINOED )

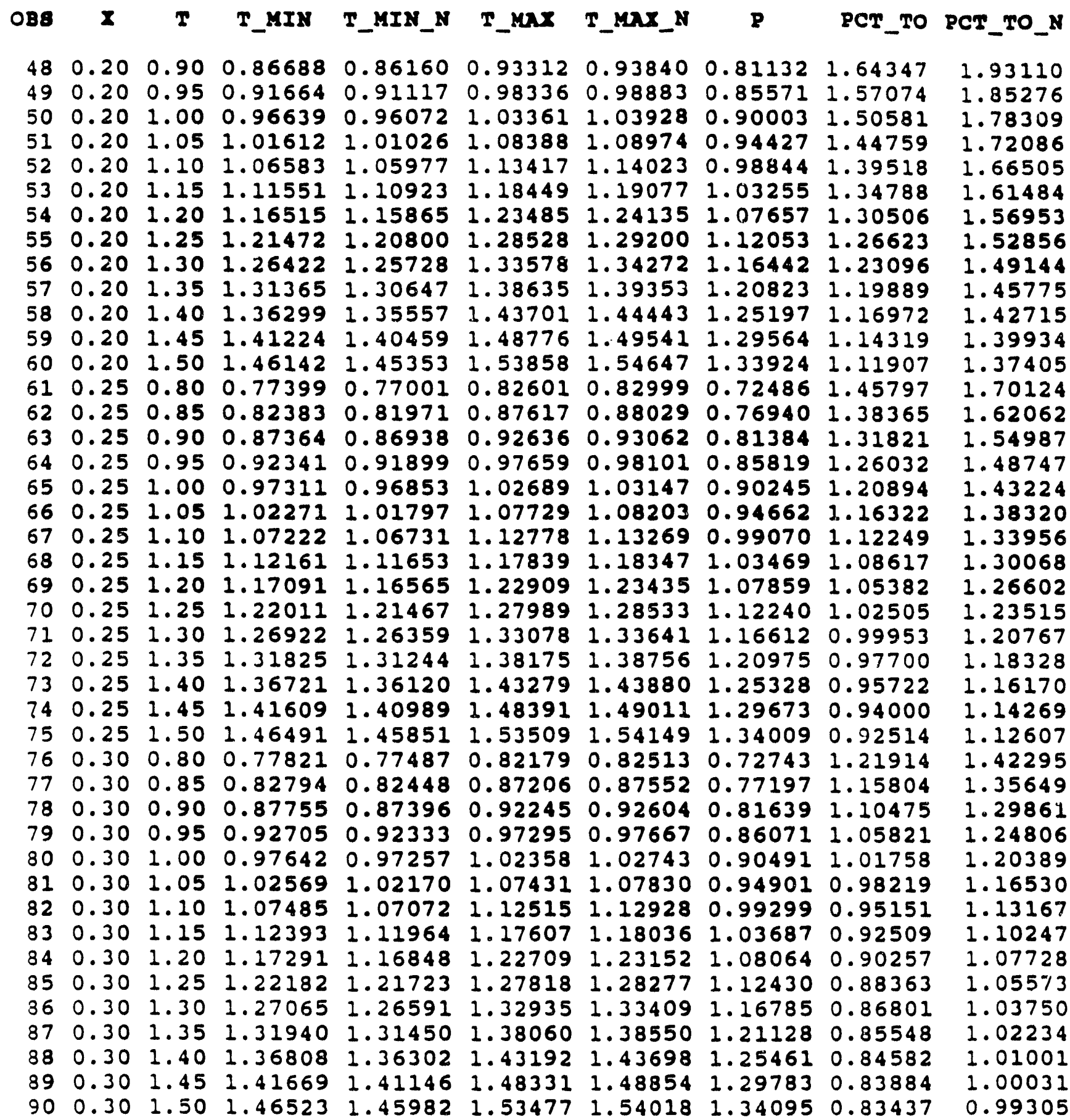


WSRC.TR-92-259

Task 92-053-1

Statistical Analysis of Test Data for APM Rod issue

May 29, 1992

Revision 0

Page 24 of 87

TABHE 4

$($ DC - APK 4)

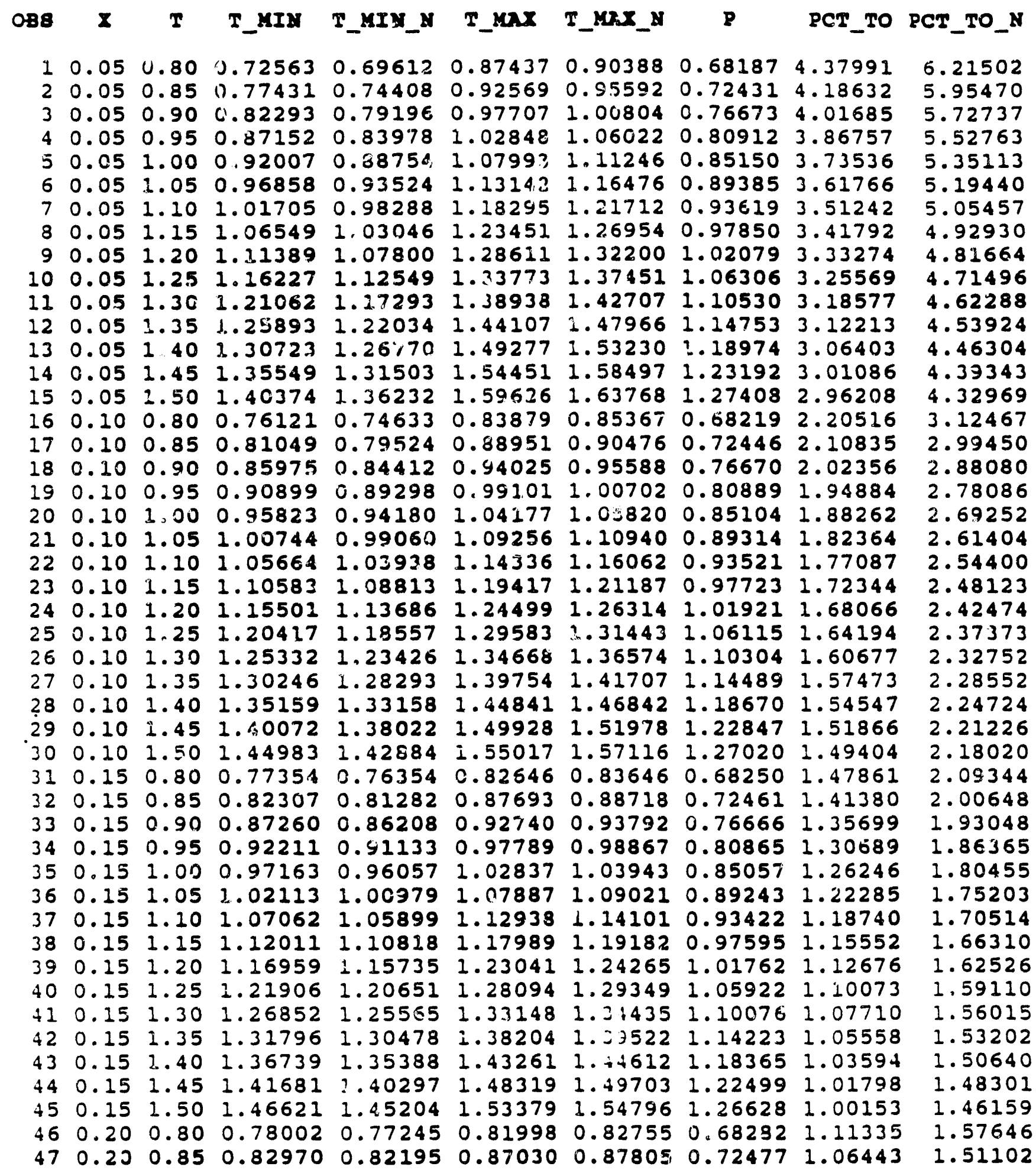


WSRC-TR-92-259

Task 92-053-1

May 29, 1992

Revision 0

Statistical Analysis of Test Data for APM Rod Issue Page 25 of 87

TABLE 4

( DC - APY 4)

( CONTINUED )

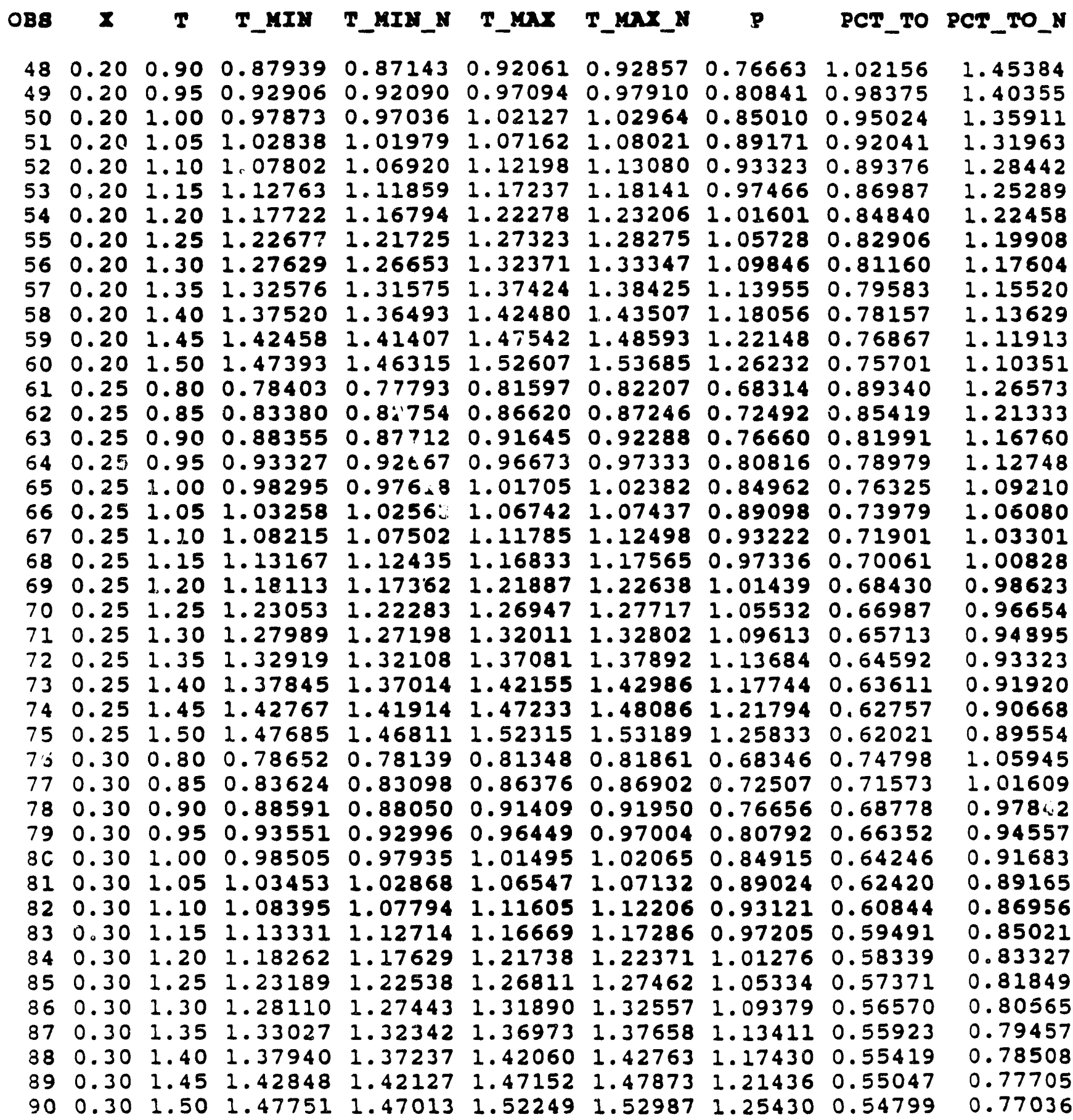


WSRC-TR-92-259

Task 92-053-1

May 29, 1992

Statistical Anglysis of Revision 0

Revision 0

TABLE 5

$($ DC - APM 5 )

\begin{tabular}{|c|c|c|c|c|c|c|c|c|c|}
\hline & $\mathbf{z}$ & I & IN & _MIN_N & $-\operatorname{sen} x$ & _Mx_N & 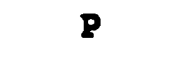 & PCT_TO & PCT_TO_N \\
\hline 1 & 05 & 0 & & & & & .68732 & 8.03927 & \\
\hline 2 & & & & & & & & & \\
\hline 3 & & & & & & & & 090 & .62334 \\
\hline 4 & & & & & .09090 & & & & 703 \\
\hline ; & & & & & & & & & 2272 \\
\hline 6 & & & & 29 & & & & & 1550 \\
\hline 7 & & 0 & & & & & & & \\
\hline 8 & & 1.1 & & & & & & & \\
\hline & & 1.2 & & & 1.39 & 1.38 & & & \\
\hline 10 & & 1.2 & 1 & & & & & & \\
\hline & 00 & 1.3 & 1.13 & & 1.4 & & 1. & & \\
\hline & .05 & 1.35 & & & & & & & \\
\hline 3 & & 1.40 & 1.22 & 1.2 & 1.57 & & 1.2 & & \\
\hline & & 1.45 & 1.27 & & 1.62 & & & & \\
\hline 1 & & 1.5 & 1.3 & 9 & 1.6 & & 1.2 & & \\
\hline & .10 & .80 & 0.73 & 01 & 0.8 & & 0.68 & & \\
\hline & & .85 & & & & & & & 97 \\
\hline 8 & 0.10 & .90 & & & & & & & \\
\hline 9 & & .95 & & & & & & & \\
\hline 20 & D & 1.0 & & & & & 8 & & 67 \\
\hline & .10 & 1.05 & & 23 & 1.1 & & & & 110 \\
\hline 22 & & & & & & & & & 907 \\
\hline 3 & & 1 & 1.0 & & & & & & 93 \\
\hline 4 & & .20 & & & & & & & \\
\hline 25 & 0 & .25 & & & 1.3 & & 1 & & 14 \\
\hline & & 9 & & & & & & & 40 \\
\hline 27 & & .35 & 1.26 & 2 & 1.4 & & 1.1 & & 31 \\
\hline 8 & & .40 & 1.3 & & & & 1.1 & & 19 \\
\hline & & 1 & 1.3 & & & & 1.2 & & \\
\hline 30 & .10 & .5 & & 31 & & 1. & 1.2 & & \\
\hline & & 0.80 & & & & & 0.6 & & \\
\hline 32 & 5 & .85 & & & & & & & \\
\hline & & & & & & & & & \\
\hline 34 & & & & & & & & & \\
\hline 35 & & 1.00 & & & & & & & \\
\hline & & & & & & & & & \\
\hline 37 & & 1.10 & & 1.0 & & & 0.9 & & \\
\hline & & & & & & & & & \\
\hline 39 & & 1.2 & & 1.1 & 1.2 & & 1.0 & & 185 \\
\hline 40 & & 1.2 & & 1.1 & 1.3 & & & & \\
\hline 41 & & & & 1.27 & 1.3 & & 1.1 & & 792 \\
\hline 42 & 0.2 & 1.35 & 1.29 & 1.28 & & & 1.1 & & \\
\hline 43 & & 1.40 & 1.338 & 1.35 & 1.46 & & 1.1 & 2.03 & 911 \\
\hline 44 & & 1.45 & & 1.37 & 1.51 & & 1.2 & & 928 \\
\hline 45 & & & & & & & & & \\
\hline 46 & & & & & & & & & \\
\hline & & & & & & & 37 & 1.95434 & 2.25288 \\
\hline
\end{tabular}


WSRC-TR-92-259

Task 92-053-1

May 29, 1992

Revision 0

Statistical Analy is of Test Data for APM hoo Issue Page 27 of 87

TABLE 5

( DC - APY 5 )

( CONTINOED )

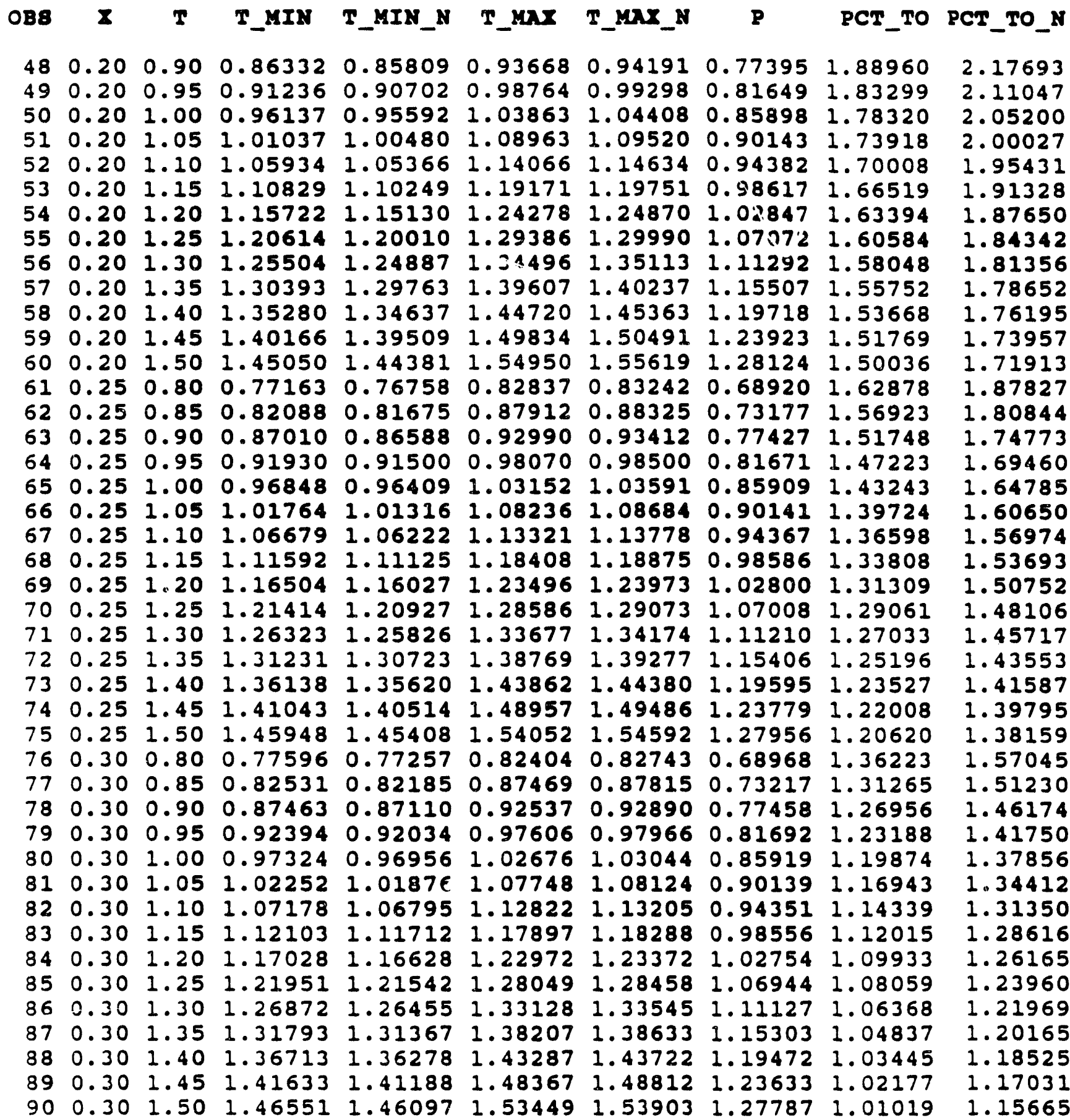


WSRC-TR-92-259

Task 92-053-1

Statistical Analysis of Test Data for APM Rod Issue

May 29, 1992

Revision 0

Page 28 of 87

TABLE 6

( DC - APM 6 )

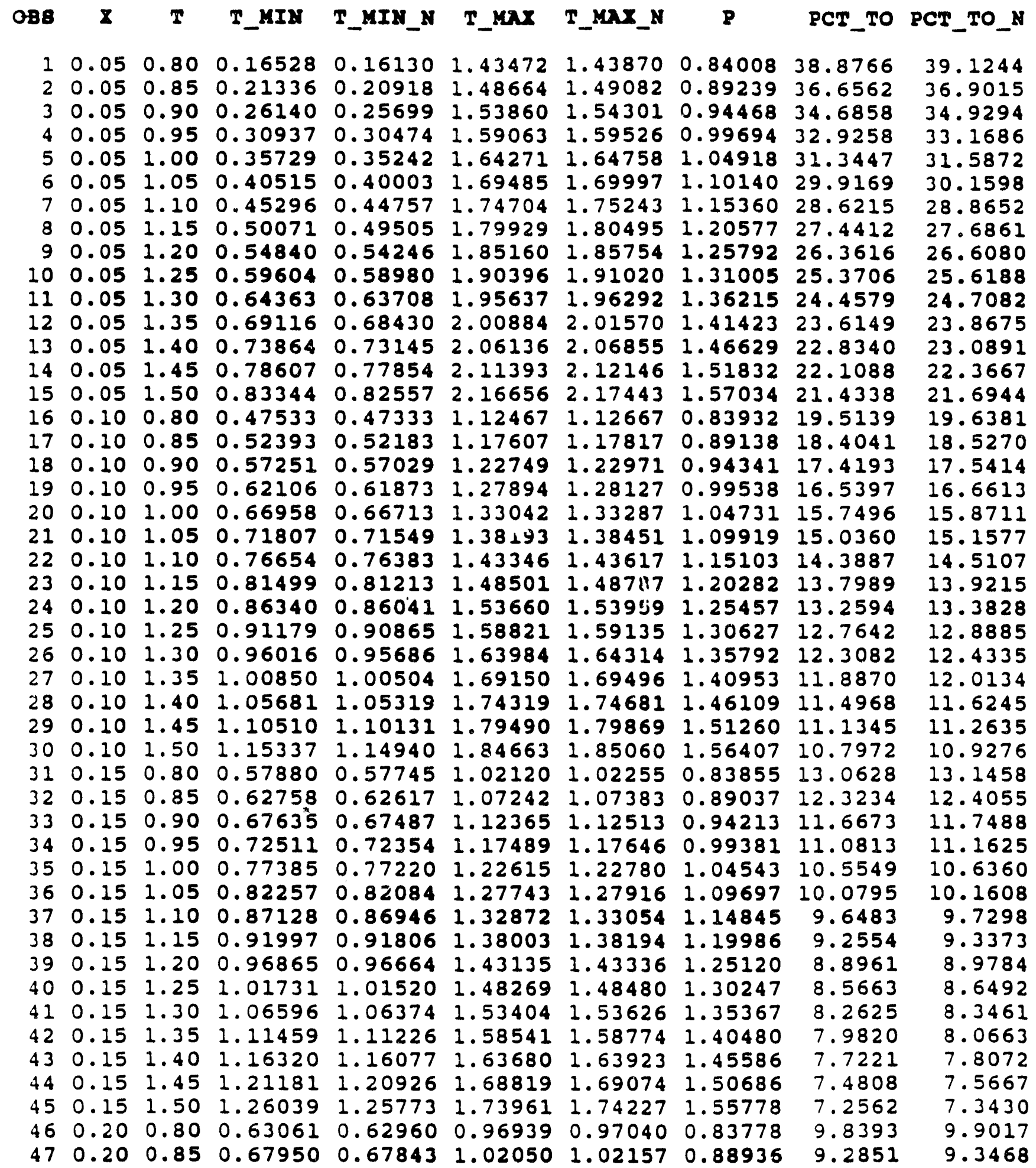


WSRC-TR-92-259

Task 92-053-1

Statistical Analysis of Test Data for APM Rod Issue

May 29, 1992

Revision 0

Page 29 of 87

TABLE 6

( DC - APM 6 )

( CONTINOED )

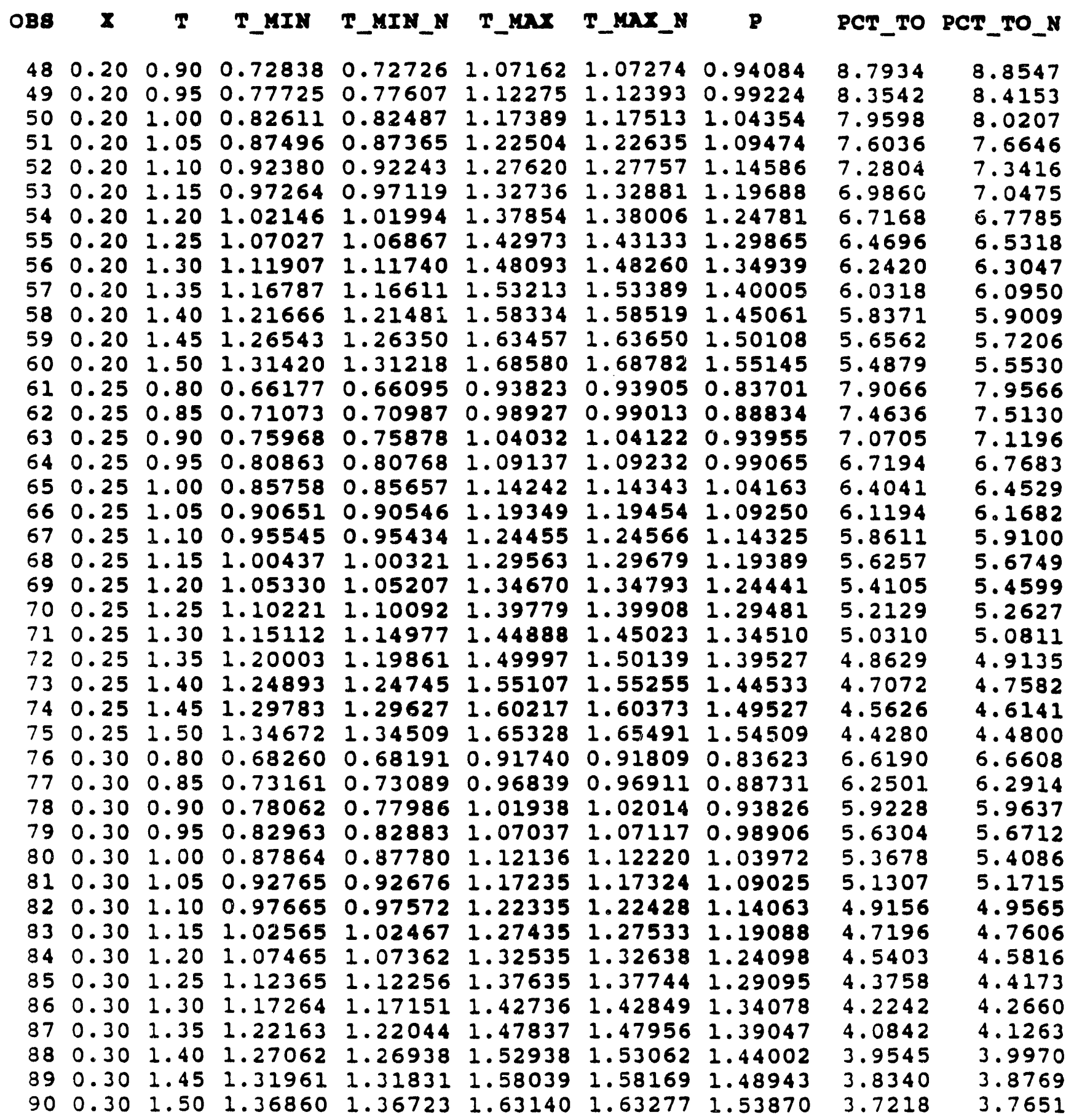


WSRC-TR-92-259

Task 92-053-1

May 29, 1992

Revision 0

Statistlagl Analysis of Test Data for APM Rod Issue Page 30 of 87

TABLE 7

$($ DC - APM 7 )

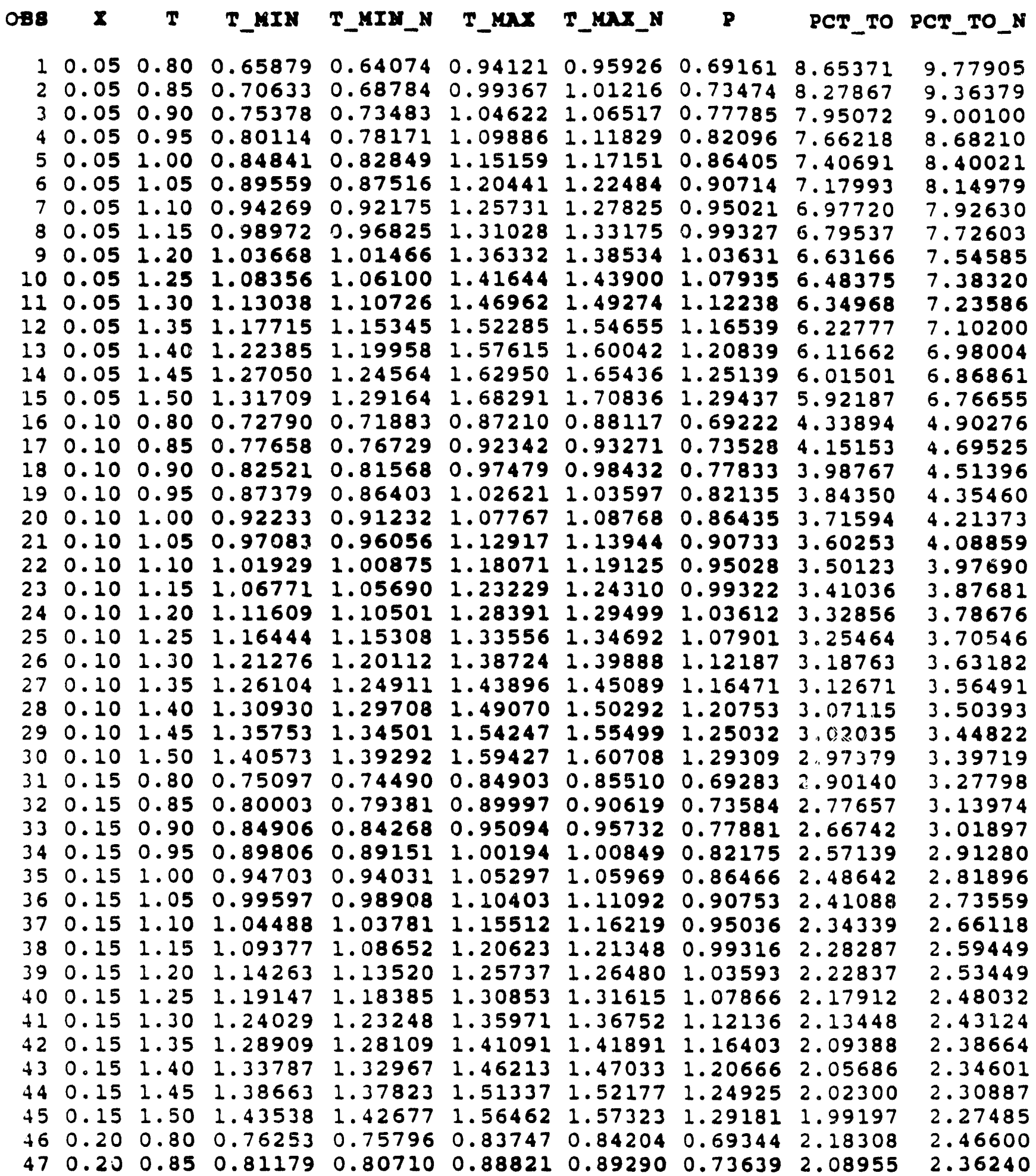


TABLE 7

$(D C-A P M 7)$

( CONTINOED )

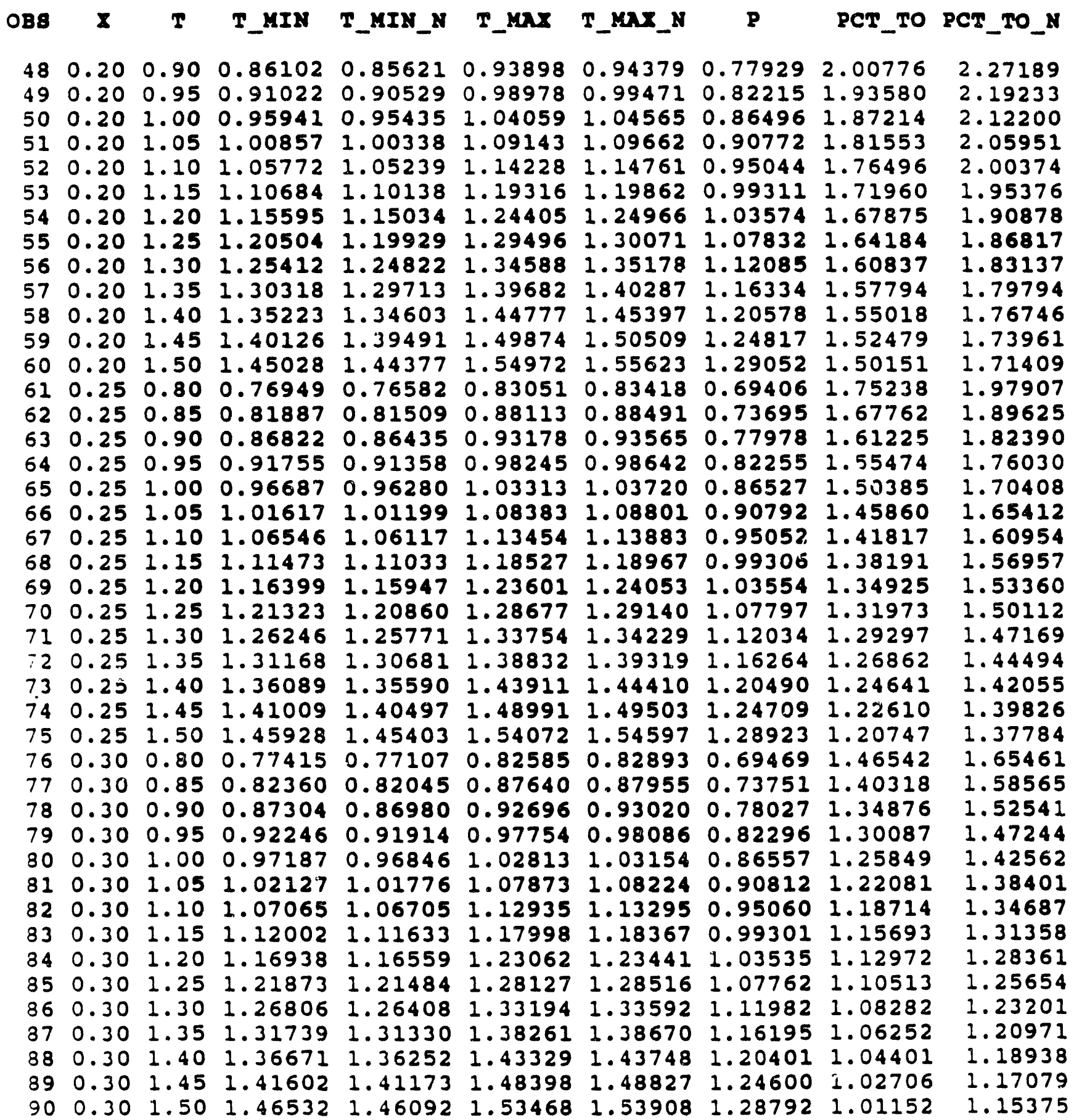


TABLE 8

( DC - APM 8 )

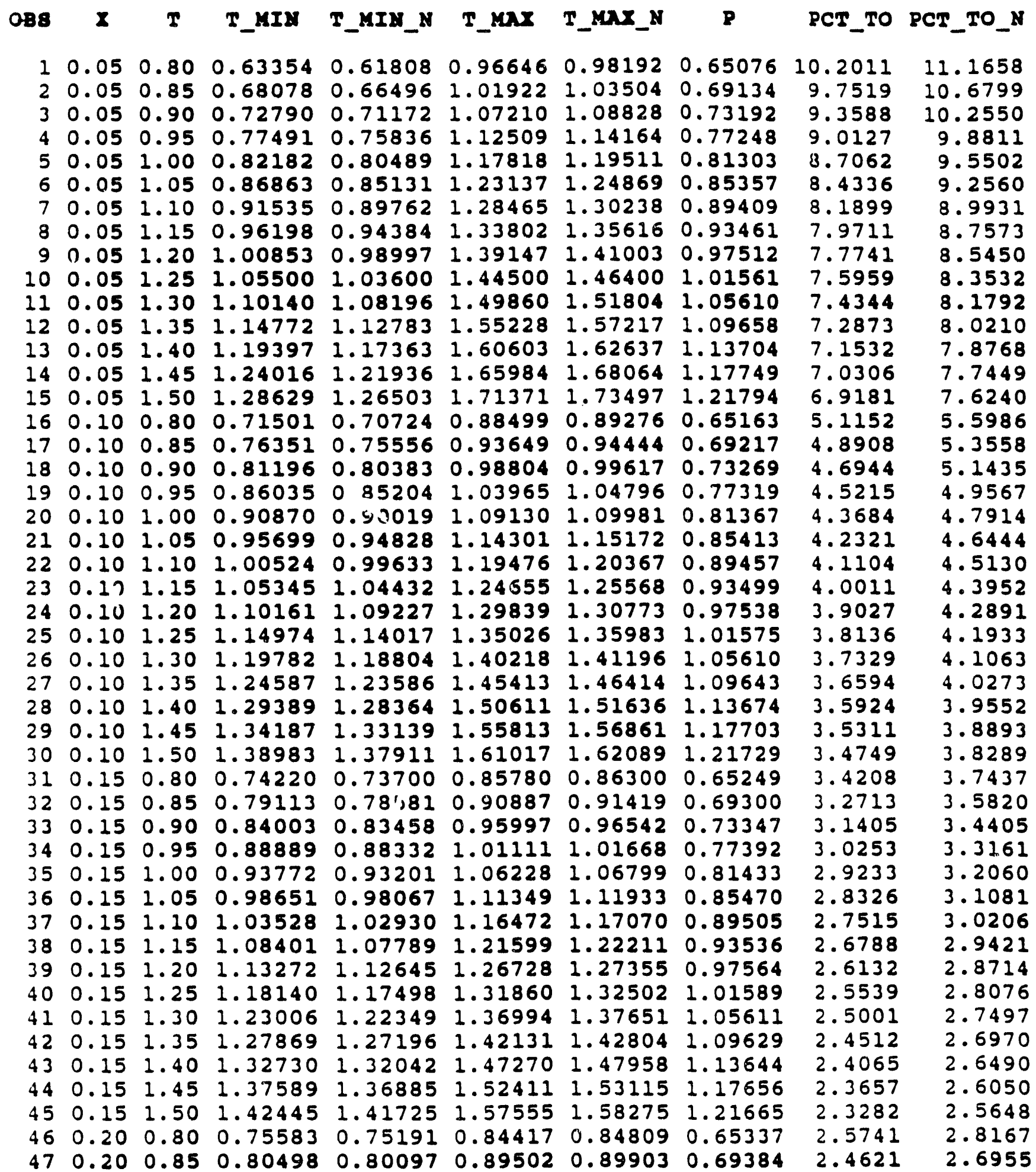


WSRC-TR-92-259

Task 92-053-1

May 29, 1992

Revision 0

Statlstlcal Analysis of Test Data for APM Red Issue Page 33 of 87

TABILE 8

( DC - APY 8 )

( CONTINOED )

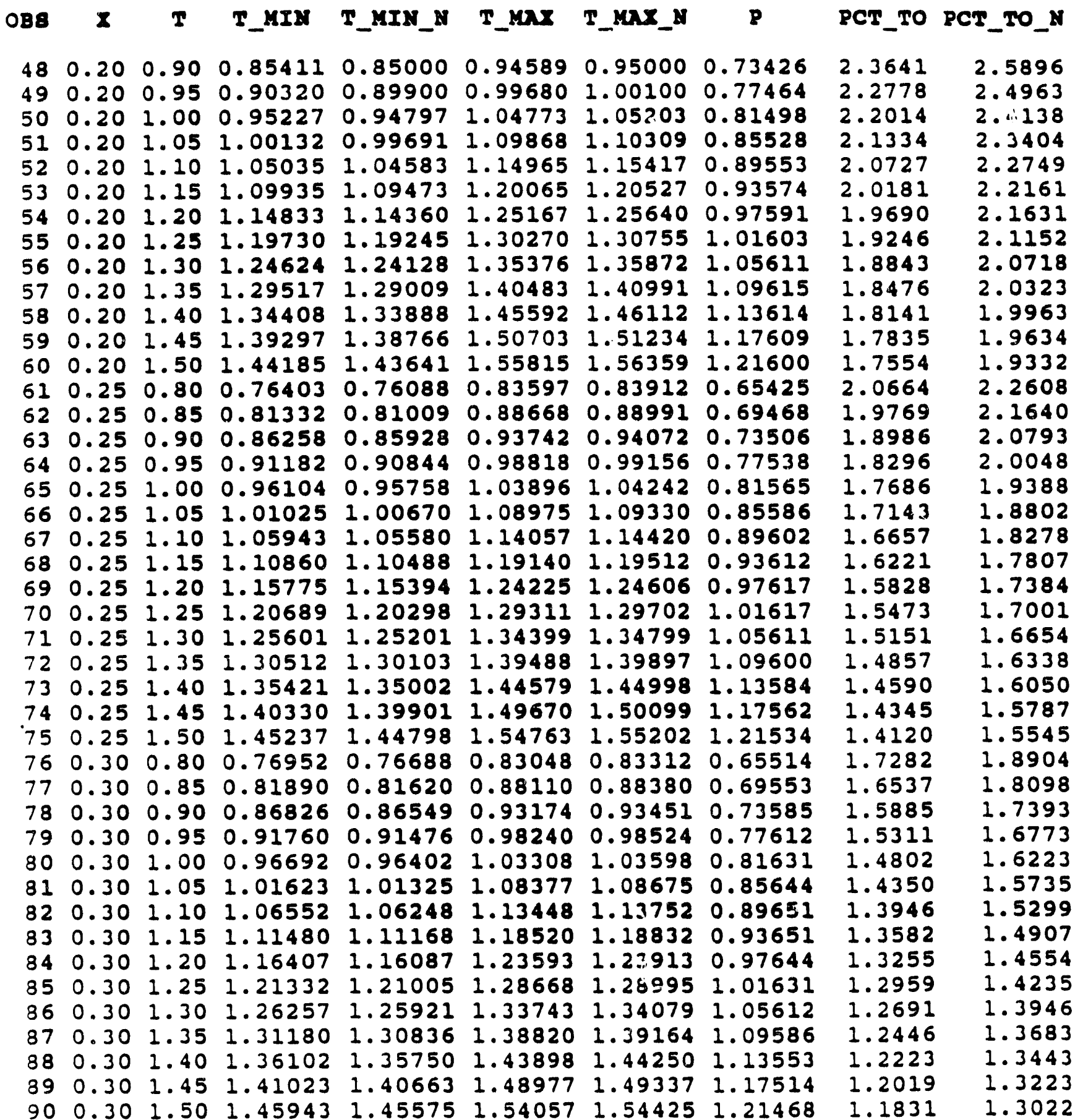


WSRC-TR-92-259

Task 92-053-1

May 29, 1992

Revision 0

Statlstical Analysis of Test Data for APM Rod issue Page 34 of 87

TABLB 9

$(\mathrm{DC}-\mathrm{APY} 9)$

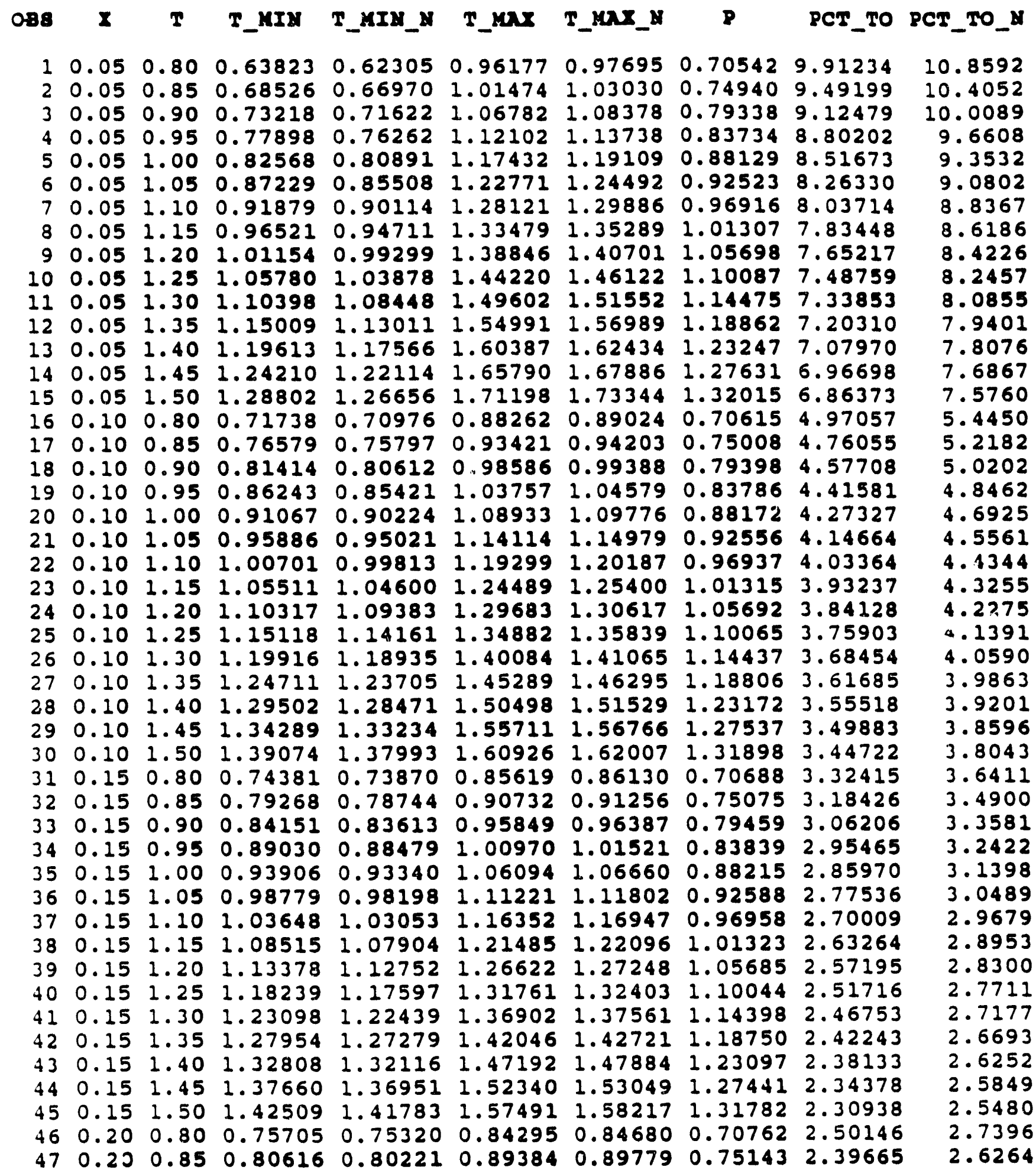


WSRC-TR-92-259

Task 92-053-1

May 29, 1992

Revision 0

Statlstlcal Analysis of Test Data for APM Rod Issue Page 35 of 87

TABLE 9

( DC - APM 9)

( CONTINOED)

\begin{tabular}{|c|c|c|c|c|c|c|c|c|c|}
\hline & $\boldsymbol{X}$ & $\mathbf{T}$ & _MIX & _MIX_N & $-\operatorname{sen} x$ & $T$ Tax_M & $\mathbf{p}$ & CT_to & CT TO \\
\hline $\begin{array}{l}48 \\
49 \\
50 \\
51 \\
52 \\
53 \\
54 \\
55 \\
56 \\
57 \\
58 \\
59 \\
60 \\
61 \\
62 \\
63 \\
64 \\
65 \\
66 \\
67 \\
68 \\
69 \\
70 \\
71 \\
72 \\
73 \\
74 \\
75 \\
76 \\
77 \\
78 \\
79 \\
80 \\
81 \\
82 \\
83 \\
84 \\
85 \\
86 \\
87 \\
88 \\
89 \\
90\end{array}$ & $\begin{array}{l}0.20 \\
0.20 \\
0.20 \\
0.20 \\
0.20 \\
0.20 \\
0.20 \\
0.20 \\
0.20 \\
0.20 \\
0.20 \\
0.20 \\
0.20 \\
0.25 \\
0.25 \\
0.25 \\
0.25 \\
0.25 \\
0.25 \\
0.25 \\
0.25 \\
0.25 \\
0.25 \\
0.25 \\
0.25 \\
0.25 \\
0.25 \\
0.25 \\
0.30 \\
0.30 \\
0.30 \\
0.30 \\
0.30 \\
0.30 \\
0.30 \\
0.30 \\
0.30 \\
0.30 \\
0.30 \\
0.30 \\
0.30 \\
0.30 \\
0.30\end{array}$ & $\begin{array}{l}0.90 \\
0.95 \\
1.00 \\
1.05 \\
1.10 \\
1.15 \\
1.20 \\
1.25 \\
1.30 \\
1.35 \\
1.40 \\
1.45 \\
1.50 \\
0.80 \\
0.85 \\
0.90 \\
0.95 \\
1.00 \\
1.05 \\
1.10 \\
1.15 \\
1.20 \\
1.25 \\
1.30 \\
1.35 \\
1.40 \\
1.45 \\
1.50 \\
0.80 \\
0.85 \\
0.90 \\
0.95 \\
1.00 \\
1.05 \\
1.10 \\
1.15 \\
1.20 \\
1.25 \\
1.30 \\
1.35 \\
1.40 \\
1.45 \\
1.50\end{array}$ & $\begin{array}{l}0.85523 \\
0.90428 \\
0.95330 \\
1.00230 \\
1.05127 \\
1.10022 \\
1.14915 \\
1.19806 \\
1.24695 \\
1.29583 \\
1.34469 \\
1.39353 \\
1.44235 \\
0.76502 \\
0.81427 \\
0.86349 \\
0.91270 \\
0.96188 \\
1.01104 \\
1.06018 \\
1.10931 \\
1.15842 \\
1.20751 \\
1.25659 \\
1.30566 \\
1.35471 \\
1.40375 \\
1.45278 \\
0.77036 \\
0.81970 \\
0.86903 \\
0.91833 \\
0.96763 \\
1.01690 \\
1.06616 \\
1.111540 \\
1.16464 \\
1.21386 \\
1.26306 \\
1.31226 \\
1.36144 \\
1.41062 \\
1.45978\end{array}$ & $\begin{array}{l}0.85118 \\
0.90012 \\
0.94903 \\
0.99792 \\
1.04677 \\
1.09561 \\
1.14442 \\
1.19321 \\
1.24198 \\
1.29073 \\
1.33946 \\
1.38817 \\
1.43686 \\
0.76193 \\
0.81109 \\
0.86023 \\
0.90935 \\
0.95844 \\
1.00751 \\
1.05656 \\
1.10560 \\
1.15461 \\
1.20360 \\
1.25258 \\
1.30155 \\
1.35049 \\
1.39943 \\
1.44835 \\
0.76777 \\
0.81704 \\
0.86630 \\
0.91553 \\
0.96475 \\
1.01394 \\
1.06313 \\
1.11229 \\
1.16144 \\
1.21058 \\
1.25970 \\
1.30881 \\
1.35790 \\
1.40699 \\
1.45606\end{array}$ & $\begin{array}{l}0.94477 \\
0.99572 \\
1.04670 \\
1.09770 \\
1.14873 \\
1.19978 \\
1.25085 \\
1.30194 \\
1.35305 \\
1.40417 \\
1.45531 \\
1.50647 \\
1.55765 \\
0.83498 \\
0.88573 \\
0.93651 \\
0.98730 \\
1.03812 \\
1.08896 \\
1.13982 \\
1.19069 \\
1.24158 \\
1.29249 \\
1.34341 \\
1.39434 \\
1.44529 \\
1.49625 \\
1.54722 \\
0.82964 \\
0.88030 \\
0.93097 \\
0.98167 \\
1.03237 \\
1.08310 \\
1.13384 \\
1.18460 \\
1.23536 \\
1.28614 \\
1.33694 \\
1.38774 \\
1.43856 \\
1.48938 \\
1.54022\end{array}$ & $\begin{array}{l}0.94882 \\
0.99988 \\
1.05097 \\
1.10208 \\
1.15323 \\
1.20439 \\
1.25558 \\
1.30679 \\
1.35802 \\
1.40927 \\
1.46054 \\
1.51183 \\
1.56314 \\
0.83807 \\
0.88891 \\
0.93977 \\
0.99065 \\
1.04156 \\
1.09249 \\
1.14344 \\
1.19440 \\
1.24539 \\
1.29640 \\
1.34742 \\
1.39845 \\
1.44951 \\
1.50057 \\
1.55165 \\
0.83223 \\
0.88296 \\
0.93370 \\
0.98447 \\
1.03525 \\
1.08606 \\
1.13687 \\
1.18771 \\
1.23856 \\
1.29942 \\
1.34030 \\
1.39119 \\
1.44210 \\
1.49301 \\
1.54394\end{array}$ & $\begin{array}{l}0.79520 \\
0.83892 \\
0.88259 \\
0.92621 \\
0.96979 \\
1.01331 \\
1.05679 \\
1.10022 \\
1.14360 \\
1.18693 \\
1.2 .3022 \\
1.27345 \\
1.31664 \\
0.70836 \\
0.75212 \\
0.79582 \\
0.83945 \\
0.89303 \\
0.92654 \\
0.97000 \\
1.01339 \\
1.05673 \\
1.10000 \\
1.14321 \\
1.18636 \\
1.22945 \\
1.27248 \\
1.31545 \\
0.70911 \\
0.75281 \\
0.79643 \\
0.83999 \\
0.88347 \\
0.92688 \\
0.97021 \\
1.01347 \\
1.05666 \\
1.09978 \\
1.14282 \\
1.18579 \\
1.22869 \\
1.27151 \\
1.31426\end{array}$ & $\begin{array}{l}2.30509 \\
2.22461 \\
2.15347 \\
2.09027 \\
2.03387 \\
1.98332 \\
1.93784 \\
1.89677 \\
1.85957 \\
1.82576 \\
1.79495 \\
1.76679 \\
1.74099 \\
2.00818 \\
1.92441 \\
1.85124 \\
1.78692 \\
1.73006 \\
1.67955 \\
1.63446 \\
1.59405 \\
1.55769 \\
1.52485 \\
1.49510 \\
1.46805 \\
1.44340 \\
1.42087 \\
1.40022 \\
1.67954 \\
1.60980 \\
1.54888 \\
1.49532 \\
1.44798 \\
1.40592 \\
1.36837 \\
1.33471 \\
1.30442 \\
1.27705 \\
1.25226 \\
1.22972 \\
1.20916 \\
1.19037 \\
1.17314\end{array}$ & $\begin{array}{l}2.5275 \\
2.4407 \\
2.3640 \\
2.2959 \\
2.2351 \\
2.1807 \\
2.1318 \\
2.0876 \\
2.0476 \\
2.0113 \\
1.9782 \\
1.9480 \\
1.9203 \\
2.1990 \\
2.1085 \\
2.0295 \\
1.9601 \\
1.8988 \\
1.8443 \\
1.7958 \\
1.7523 \\
1.7131 \\
1.6778 \\
1.6458 \\
1.6168 \\
1.5903 \\
1.5661 \\
1.5440 \\
1.8388 \\
1.7634 \\
1.6977 \\
1.6399 \\
1.5888 \\
1.5435 \\
1.5030 \\
1.4668 \\
1.4342 \\
1.4048 \\
1.3781 \\
1.3539 \\
1.3318 \\
1.3116 \\
1.2932\end{array}$ \\
\hline
\end{tabular}


TABLE 10

$($ AC - APY 1$)$

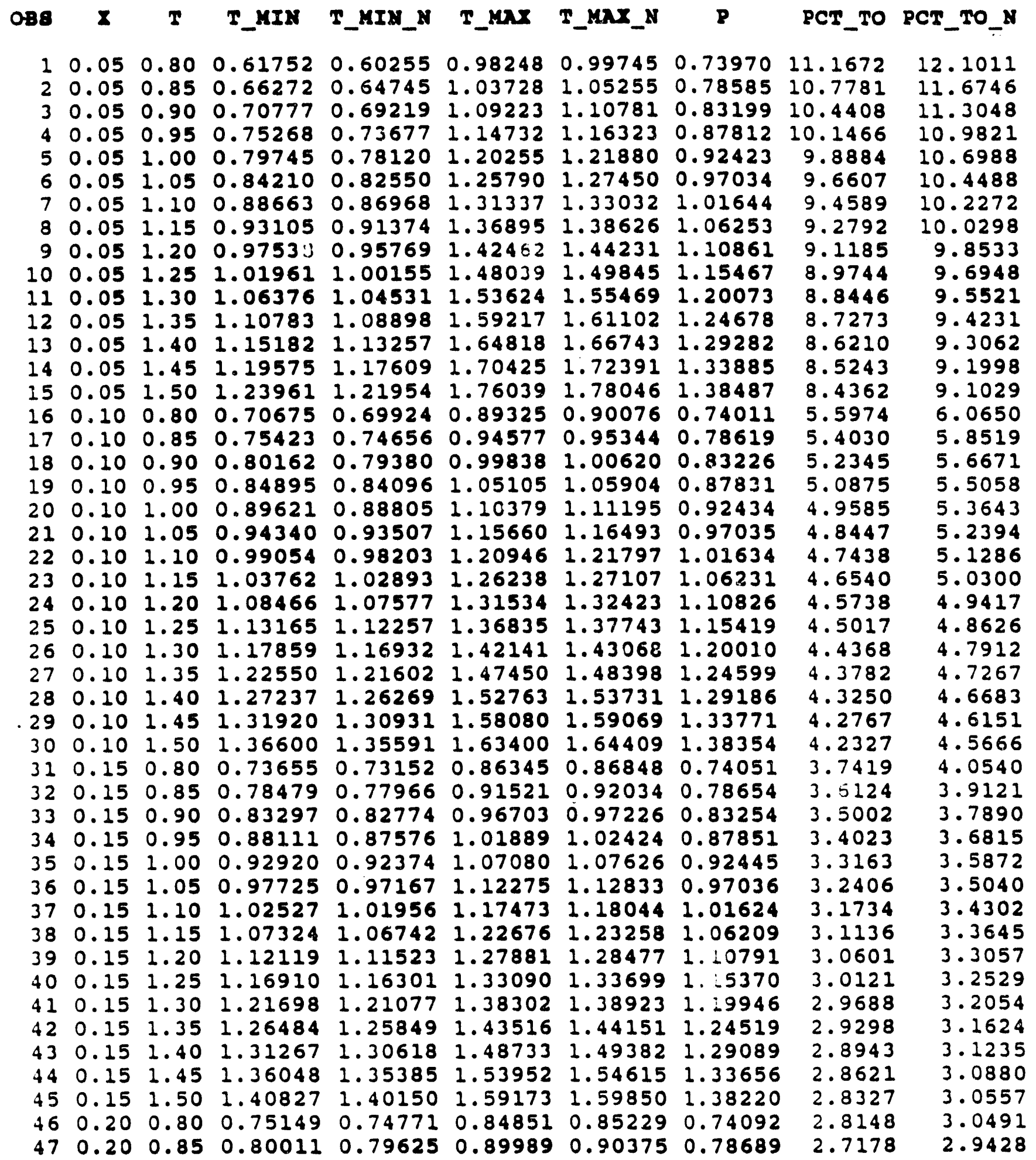




\author{
TABIE 10
}

$(A C-A P Y 1)$

( CONTINOED )

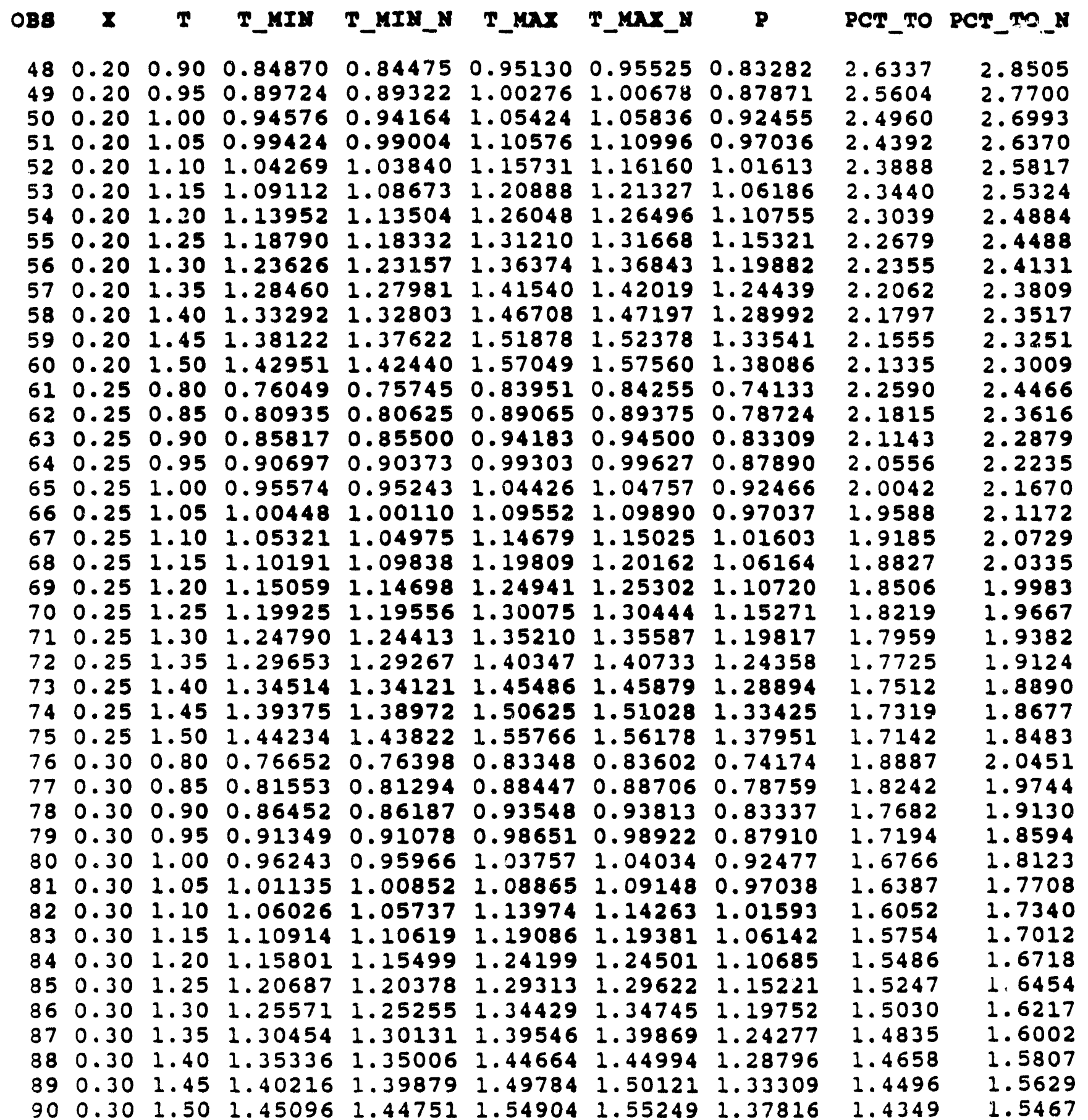


WSRC-TR-92-259

Task 92-053-1

May 29, 1992

Statistical Analysis of Revision 0

Statistical Analysis of Test Data for APM Rod Issue Page 38 of 87

TABLE 11

$(A C-A P M 2)$

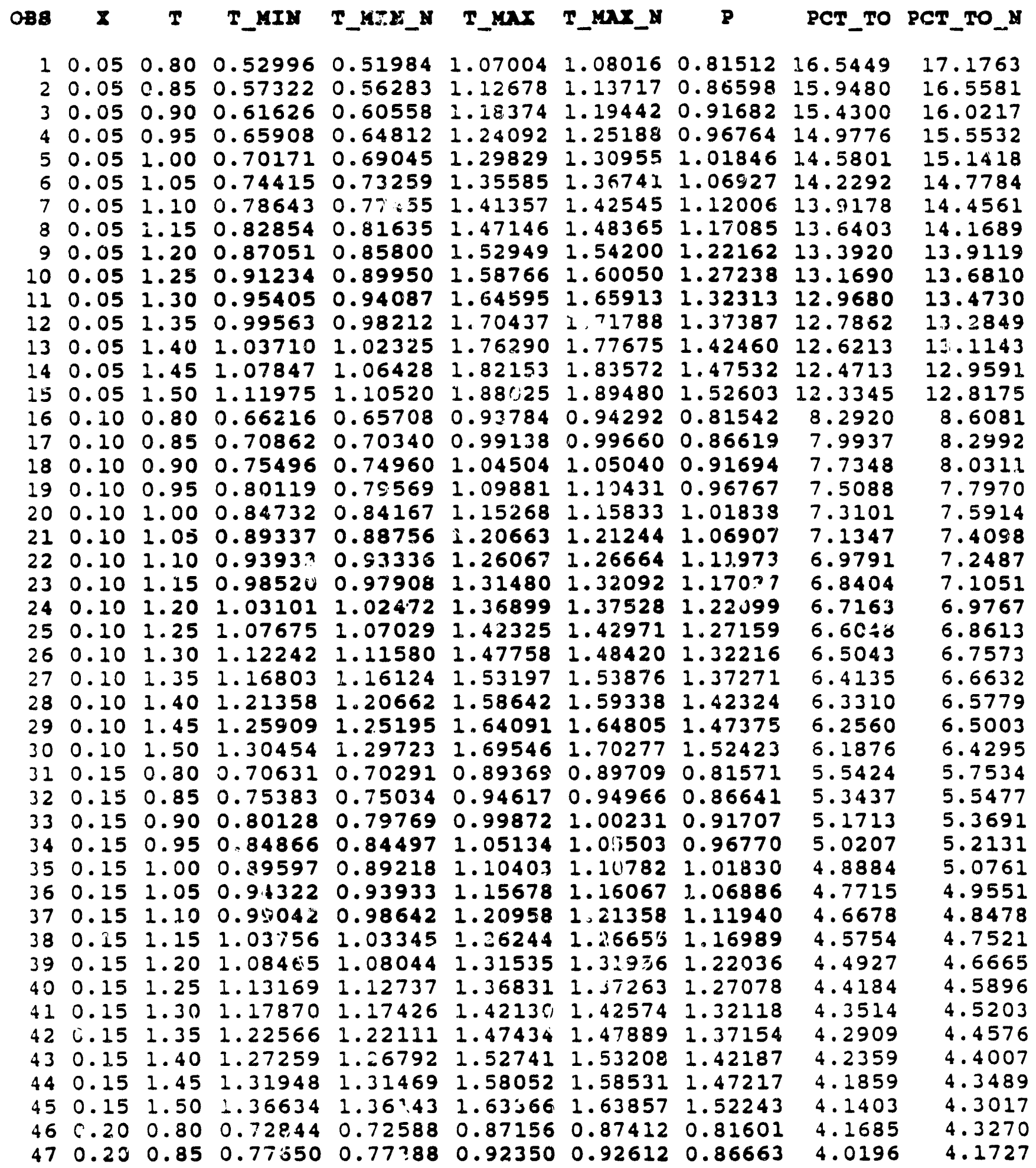


WSRC-TR-92-259

Task 92-053-1

Task 92-053-1
Statistical Analysis of Test Data for APM Rod Issue Page 39 of 87

May 29, 1992

TABLB 11

$(A C-A P H 2)$

( CONTINOED )

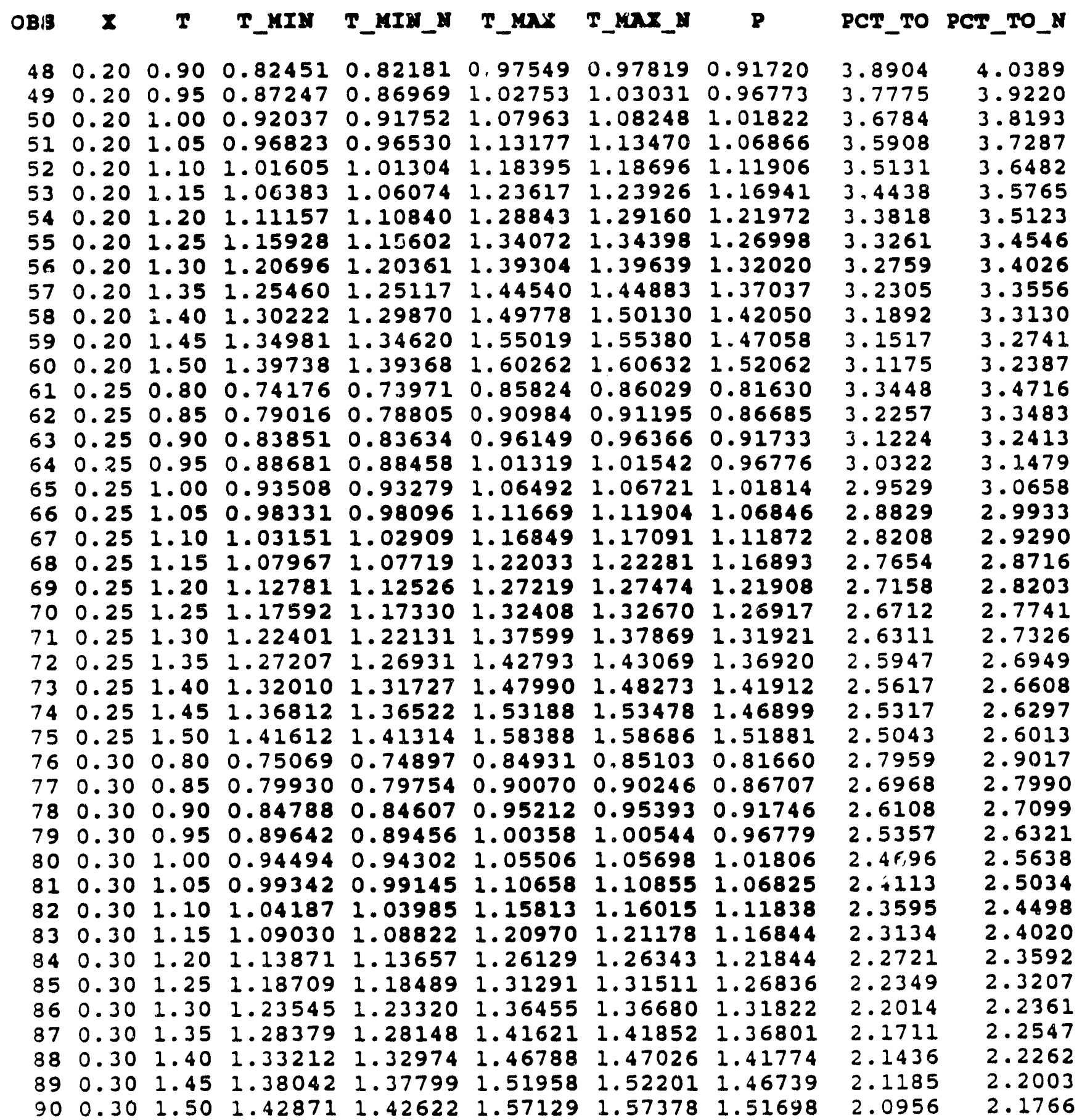


WSRC-TR-92-259

Task 92-053-1

Statistical Analysis of Test Data for APM Red Issue

May 29, 1992

Revision 0

Page 40 of 87

TABIR 12

$(A C-A P M \quad 3)$

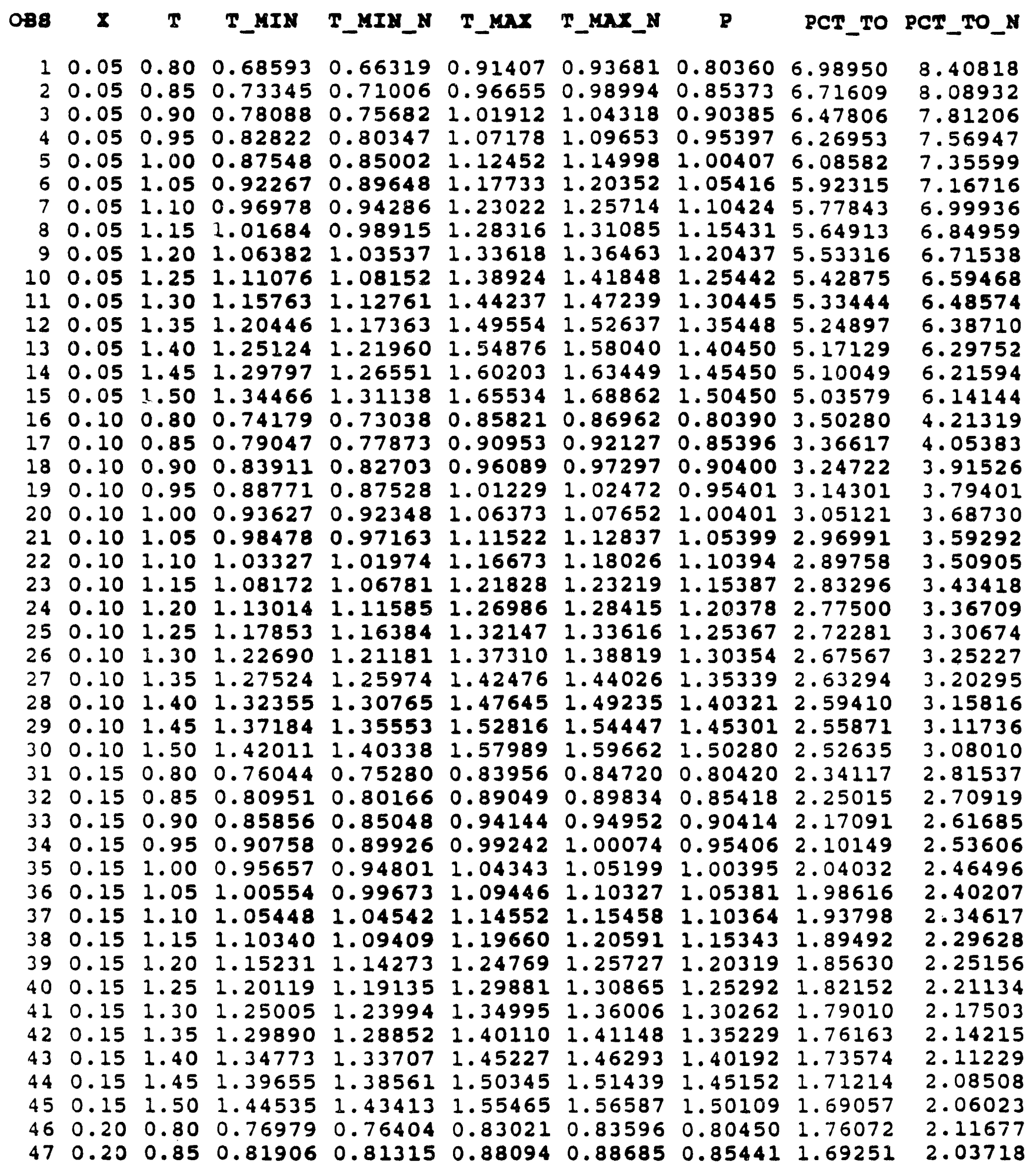


WSRC-TR-92-259

Task 92-053-1

Statistical Analysis of Test Data for APM Rod Issue

May 29, 1992

Revision 0

Page 41 of 87

TABLE 12

$(A C-A P M 3)$

( CONTINOED )

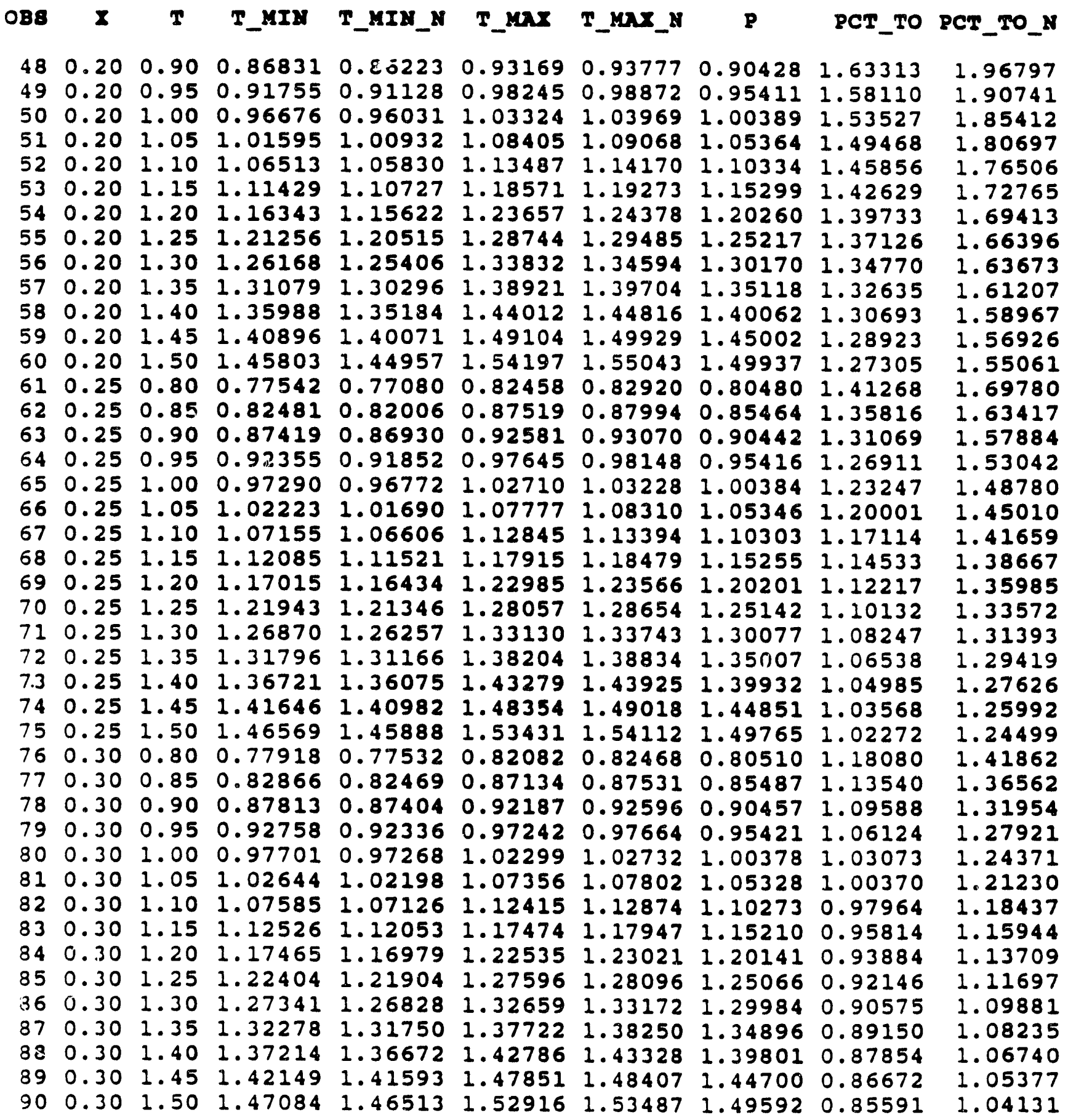


TABLE 13

\author{
$(A C-A P M \quad$ \& $)$
}


WSAC-TR-92-259

Task 92-053-1

Statistical Analysis of Test Data for APM Rod Issue Page 43 of 87

May 29, 1992
Revision 0
Page 43 of 87

TARLE 13

$(A C-A P Y 4)$

( CONTINOED )

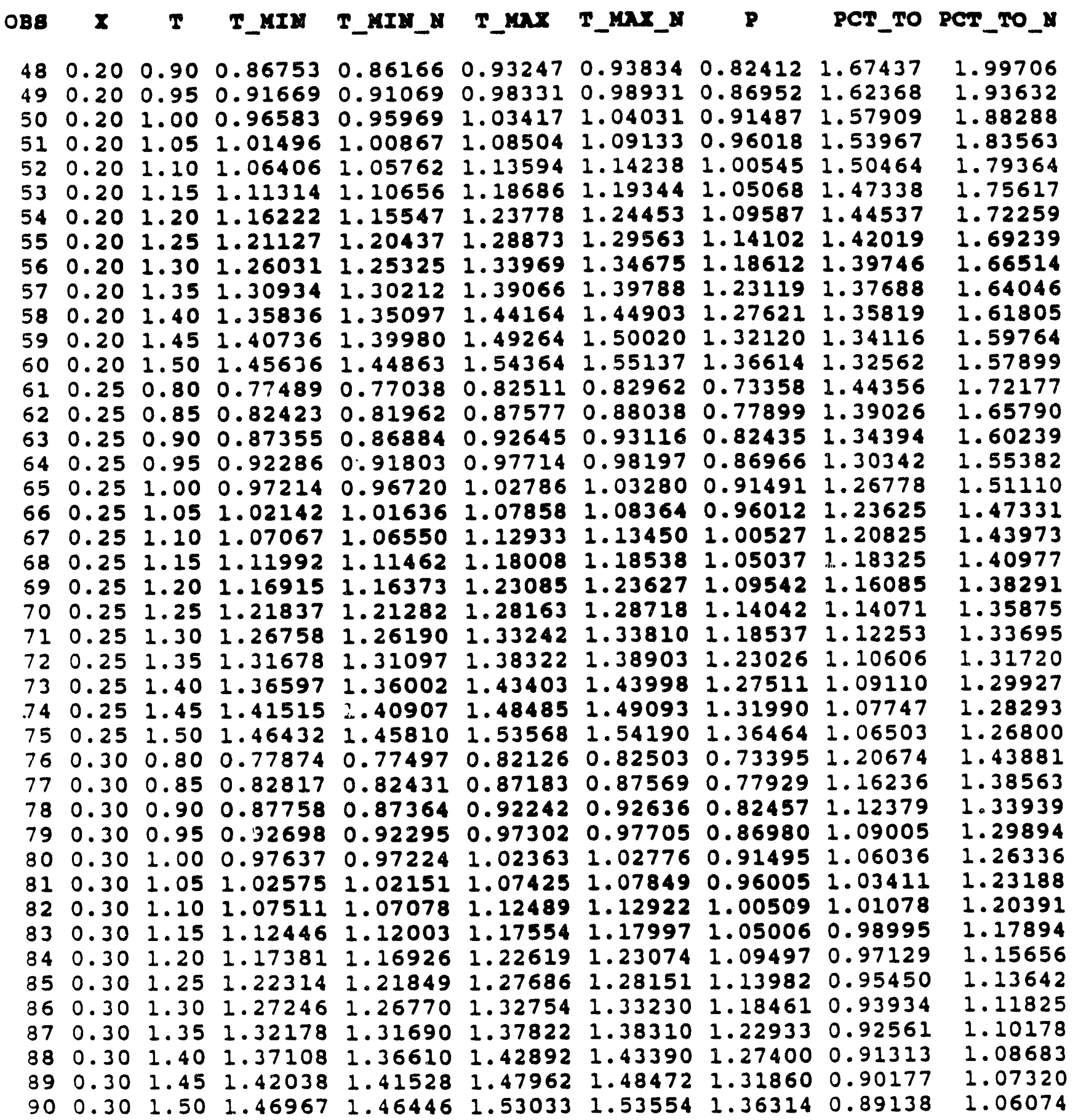


WSAC-TR-92-259

Task 92-053-1

Statlstical Analysis of Test Data for APM Rod Issue

May 29, 1992

Revision 0

Page 44 of 87

TABLE 14

$($ AC - APY 5$)$

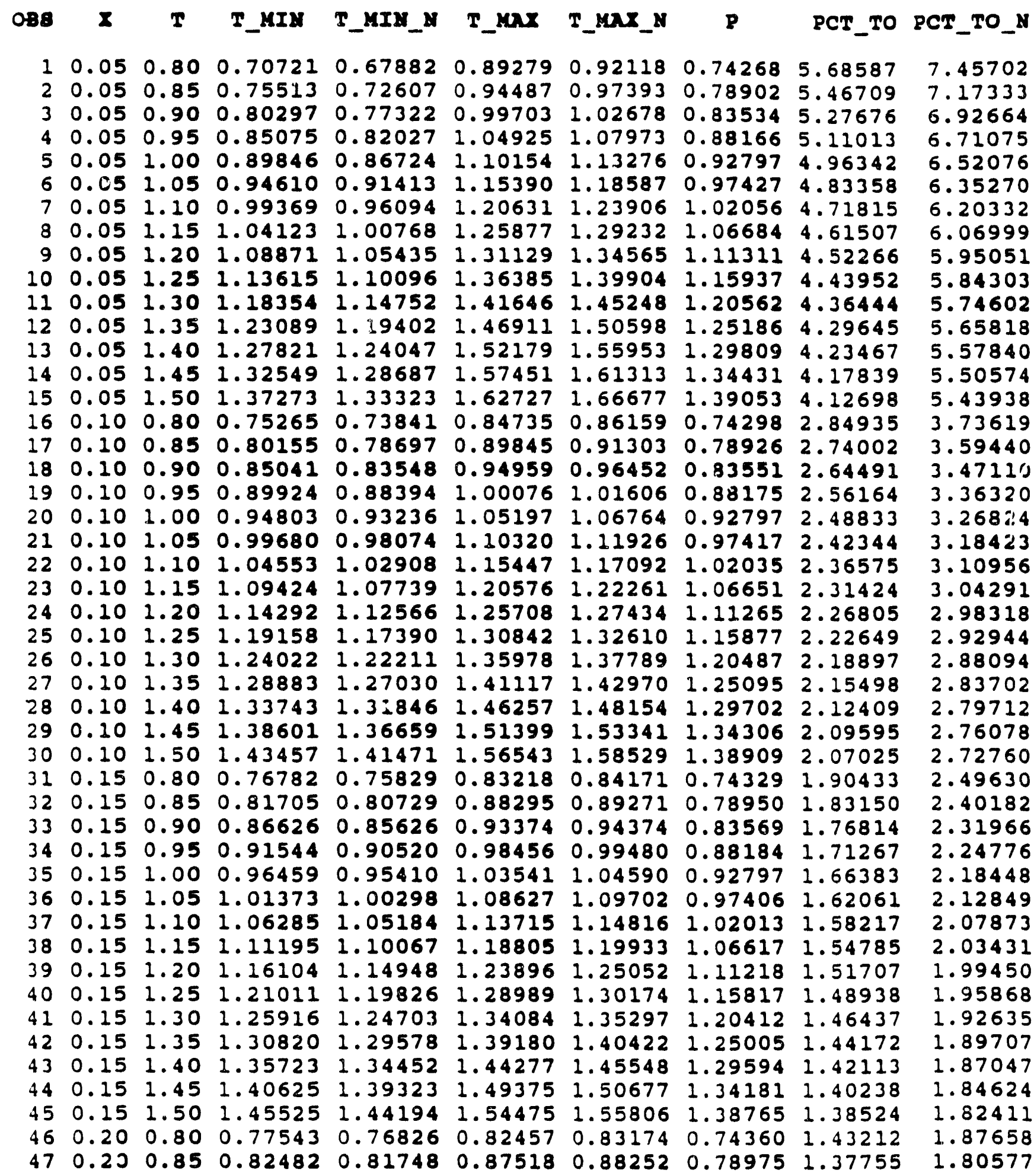


WSRC-TR-92-259

Task 92-053-1

Statistlcal Analysis of Test Data for APM Rod Issue

May 29, 1992

Revision 0

Page 45 of 87

TABIF 11

$(\mathrm{AC}-\mathrm{APM} 5)$

( CONTINOED )

\begin{tabular}{|c|c|c|c|c|c|c|c|c|c|}
\hline 98 & $\mathbf{z}$ & $\mathbf{T}$ & T_MIN & T_MIN_N & T_max & T_Manx_N & $\mathbf{P}$ & PCT_TO & PCT_TO_N \\
\hline & & & & & & & & & \\
\hline & & & & & & & & & \\
\hline & & & & 0 & .027 & & & & 284 \\
\hline & & & & 13 & & 87 & 996 & & 087 \\
\hline & & 1 & & 25 & 45 & 75 & 1.0 & & 357 \\
\hline & & 1.15 & 1.12085 & 1.11234 & 1.17915 & 8766 & 1.06 & 1.164 & \\
\hline & & & 1.17013 & 1.16142 & 1.22987 & 58 & 1.1 & $1.14:$ & \\
\hline & & 1.2 & 1. & 1.21 & 1.28 & 1. & 1.2 & & \\
\hline & 20 & 1.3 & 1.26 & 1.25 & 1.33132 & 1.3 & 1.2 & 1.1 & \\
\hline & .20 & 1.35 & & 1.30 & & & 1.2 & & \\
\hline & & 1.40 & $1.36^{\circ}$ & 1.35 & 1.43282 & & 1.25 & & 0738 \\
\hline 5 & 20 & 1.45 & 1.41 & 1.40 & 1.48 & 1.4 & 1.3 & & \\
\hline 60 & 20 & 1.5 & $1.46 !$ & 1.45 & 1 & 1. & 1.3 & 1.0 & \\
\hline & .25 & 0.80 & 001 & 0.77 & 0.81999 & 75 & 0.7 & 1.1 & \\
\hline & & 0.85 & & 0.82 & $0.87 \mathrm{C}$ & & 0.7 & & 328 \\
\hline 63 & .25 & 0.90 & & 94 & 0.92101 & 06 & 0.8 & & \\
\hline 64 & .25 & 0.95 & 0.5 & 226 & 0.97 & & 0.8 & $1 . c$ & \\
\hline 6 & 25 & 1.00 & 0.97 & 0.97 & 1.022 & 1.02844 & 0.9 & 1.0 & \\
\hline & 25 & 1.05 & 1.0 & 1.02 & 1.07264 & 1.07915 & 0.97 & & \\
\hline & & 1.10 & 1.07 & 1.0 & $1.12=$ & & 1.0 & & \\
\hline 68 & & 1.15 & 1.12 & 1.11 & 1.173 & 1.18 & 1.0 & & \\
\hline 69 & 25 & 1.20 & 1.17562 & 1.16 & 1.22438 & 1.23 & 1.1 & 0.9 & \\
\hline 7 & 0.25 & 1.25 & 1.22502 & 1.23 & 1.27498 & 1.28215 & 1.1 & $0 . \varsigma$ & \\
\hline 7 & 0.25 & 1.30 & 1.27442 & 1.26 & 1.32558 & 293 & & & \\
\hline 72 & 0.25 & 1.35 & 1.32380 & 1.31628 & 1.37620 & 1.38372 & 1.24822 & 0.87177 & 1.14563 \\
\hline 73 & & 1.40 & 1.37 & 1.30 & 1.426 & 453 & 1.2 & & \\
\hline 74 & 0.25 & 1.45 & 1.42256 & 1.4 & 1.47 & 534 & 1.3 & 0.8 & $\$ 11$ \\
\hline 75 & 0.25 & 1.50 & 1.47 & 1.4 & 1.528 & 616 & 1.3 & 0.8 & \\
\hline 76 & 0.30 & 0.80 & 0.78307 & & 0.816 & 74 & 422 & 0. & 721 \\
\hline 77 & 0.30 & 0.85 & 0.83 & 0.8 & & 229 & 0.7 & 0. & 1.2 \\
\hline 78 & 0.30 & & 0 . & $0.8^{\circ}$ & & 286 & 0.8 & 242 & 1.2 \\
\hline 79 & 0.30 & & & 0.9 & 0 & 344 & 211 & 0.8 & 3314 \\
\hline 80 & 0.30 & 1.00 & & & & 104 & 0.9 & $0 . \varepsilon$ & 1 \\
\hline 8 & 0.30 & 1.05 & 1.03079 & 1.0 & 1.069 & 65 & 0.9 & 0.8 & 1.0 \\
\hline 82 & 0.30 & 1.10 & 1.08030 & 1.07472 & 1.119 & 1.12 & 1.01949 & 0.7 & 1.0 \\
\hline 83 & 0.30 & 1.15 & 1.12981 & 1.12409 & 1.17019 & 1.17591 & 1.06517 & $0.7 \varepsilon$ & 2652 \\
\hline & & 1.20 & 1.17930 & 1.17344 & 1.22070 & 1.22656 & 1.11079 & & \\
\hline 8 & 0.30 & 1.25 & 1.22879 & 1.22278 & & 1.27722 & 1.1 & 0.7 & 0 \\
\hline 86 & 0.30 & 1.30 & 1.27827 & 1.27212 & 1.321 & 1.32788 & 1.20185 & 0.7 & 0.97254 \\
\hline 87 & 0.30 & 1.35 & 1.32775 & 1.32144 & 1.372 & 1.37856 & 1.24730 & 0.72943 & 0.45789 \\
\hline 88 & 0.30 & 1.40 & 1.37722 & 1.37076 & 1.422 & 1.42924 & 1.29269 & 0.71912 & 0.94457 \\
\hline $8 \subseteq$ & 0.30 & 1.45 & 1.42668 & 1.42007 & 1.47332 & 1.47993 & 1.33802 & & 0.93243 \\
\hline & & & & 1.46937 & & 1.53063 & 1.38329 & 0.70114 & 0.92134 \\
\hline
\end{tabular}


WSRC-TR-92-259

Task $92-053-1$

Statlstlcal Analysis of Test Data for APM Red issue
May 29, 1992

Revision 0

\author{
TABLE 15
}

$(A C-A P Y 6)$

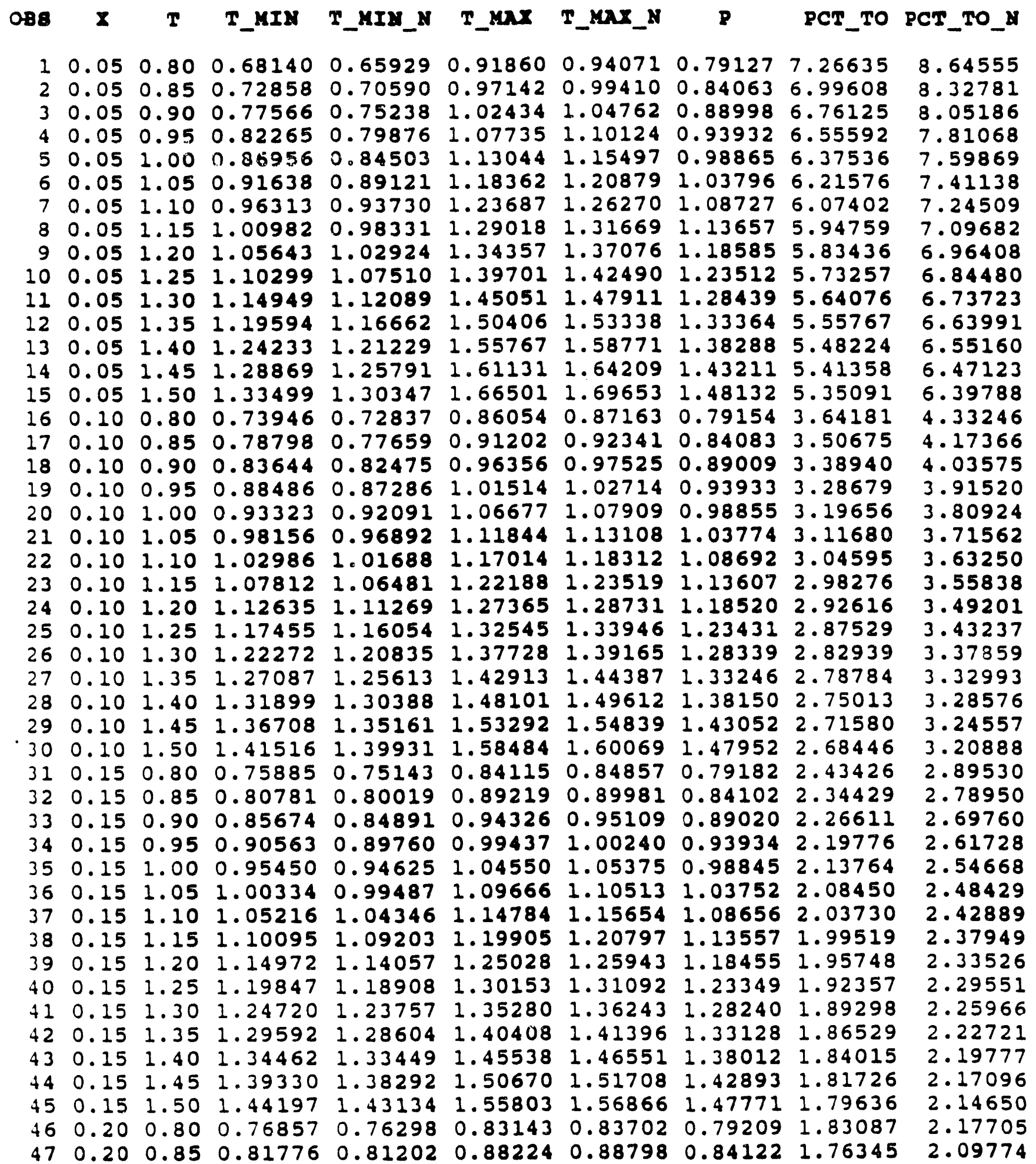


WSRC-TR-92-259

Task 92-053-1

Statistlcal Analysis of Test Data for APM Red Issue Page 47 of 87

May 29, 1992

Revision 0

TAPLE 15

$($ AC - APM 6$)$

( CONTIMUED )

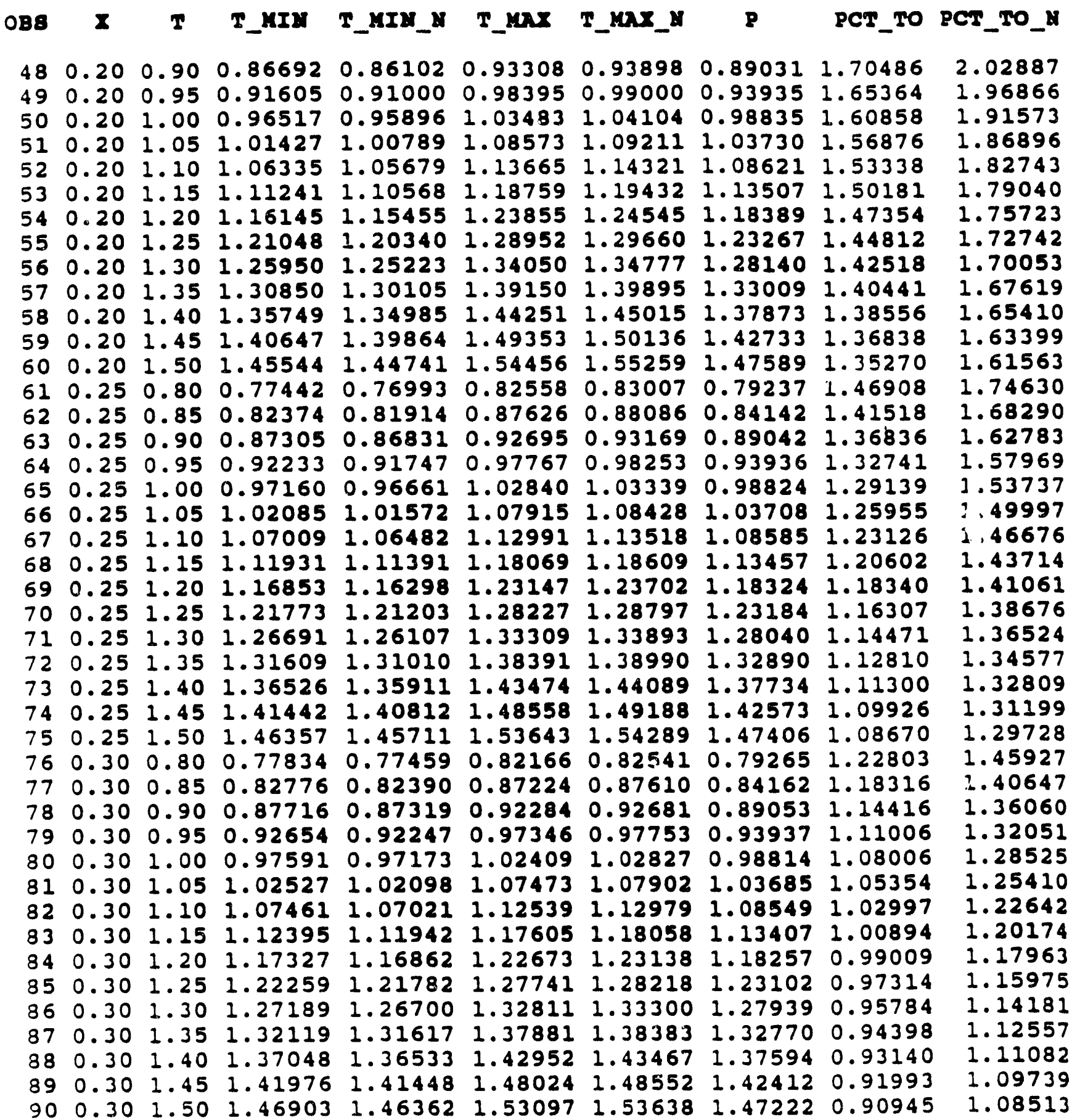


TABLE 26

\author{
$(\operatorname{AC}-\operatorname{APX} 7)$
}

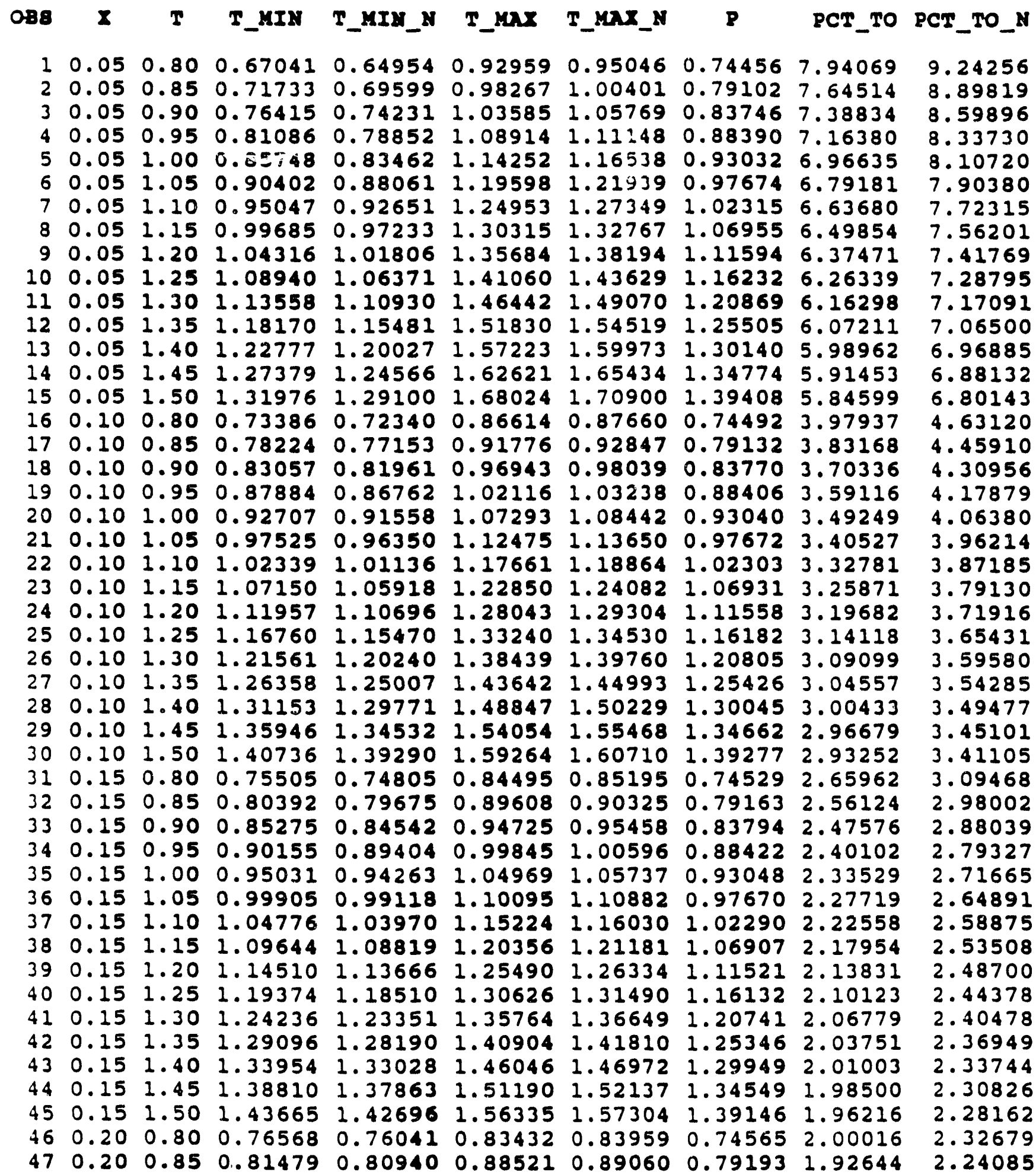


WSRC-TR-92-259

Task 92-053-1

May 29, 1992

Statistlcal Analysis of Test Data for APM Rod Issue Page 49 of 87

Revision 0

TARLE 16

$(\mathrm{AC}-\mathrm{APM} 7)$

( CONTINOED )

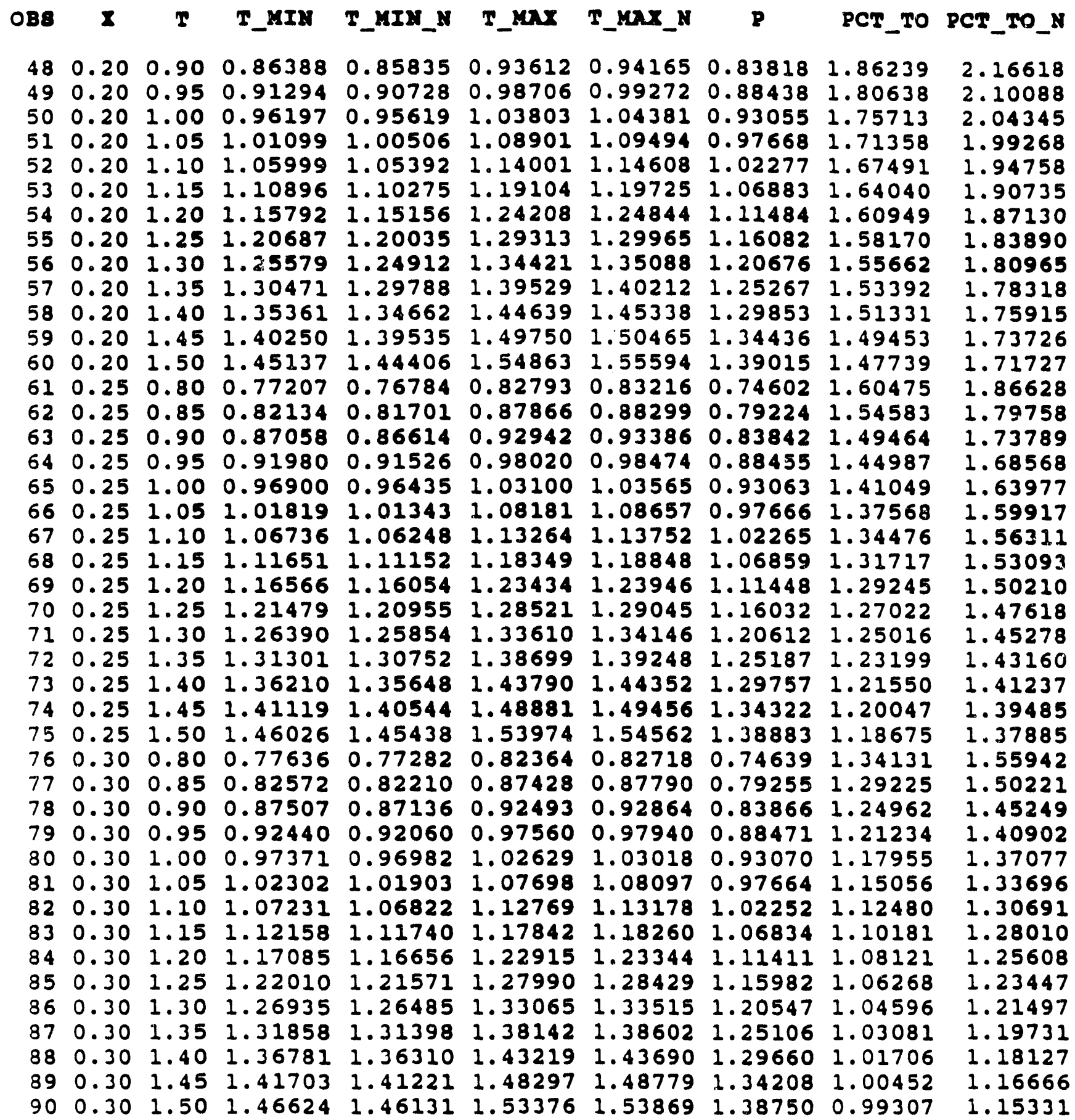


WSRC-TR-92-259

Task 92-053-1

Statistical Analysis of Test Data for APM Rod Issue

May 29, 1992

Revision 0

Page 50 of 87

TABLB 17

$(A C-A P M B)$

\begin{tabular}{|c|c|c|c|c|c|c|c|c|c|}
\hline $\mathbf{B 8}$ & $\mathbf{z}$ & $\mathbf{T}$ & C_MIX & YTI & $1 \ln x$ & C_cosx_y & $\mathbf{P}$ & РCT TO & PCT to y \\
\hline $\begin{array}{r}1 \\
2 \\
3 \\
4 \\
5 \\
6 \\
7 \\
8 \\
9 \\
10 \\
11 \\
12 \\
13 \\
14 \\
15 \\
16 \\
17 \\
18 \\
19 \\
20 \\
21 \\
22 \\
23 \\
24 \\
25 \\
26 \\
27 \\
28 \\
29 \\
30 \\
31 \\
32 \\
33 \\
34 \\
35 \\
36 \\
37 \\
38 \\
39 \\
40 \\
41 \\
42 \\
43 \\
44 \\
45 \\
46 \\
47\end{array}$ & $\begin{array}{l}0.05 \\
0.05 \\
0.05 \\
0.05 \\
0.05 \\
0.05 \\
0.05 \\
0.05 \\
0.05 \\
0.05 \\
0.05 \\
0.05 \\
0.05 \\
0.05 \\
0.05 \\
0.10 \\
0.10 \\
0.10 \\
0.10 \\
0.10 \\
0.10 \\
0.10 \\
0.10 \\
0.10 \\
0.10 \\
0.10 \\
0.10 \\
0.10 \\
0.10 \\
0.10 \\
0.15 \\
0.15 \\
0.15 \\
0.15 \\
0.15 \\
0.15 \\
0.15 \\
0.15 \\
0.15 \\
0.15 \\
0.15 \\
0.15 \\
0.15 \\
0.15 \\
0.15 \\
0.20 \\
0.20\end{array}$ & $\begin{array}{l}0.80 \\
0.85 \\
0.90 \\
0.95 \\
1.00 \\
1.05 \\
1.10 \\
1.15 \\
1.20 \\
1.25 \\
1.30 \\
1.35 \\
1.40 \\
1.45 \\
1.50 \\
0.80 \\
0.85 \\
0.90 \\
0.95 \\
1.00 \\
1.05 \\
1.10 \\
1.15 \\
1.20 \\
1.25 \\
1.30 \\
1.35 \\
1.40 \\
1.45 \\
1.50 \\
0.80 \\
0.85 \\
0.90 \\
0.95 \\
1.00 \\
1.05 \\
1.10 \\
1.15 \\
1.20 \\
1.25 \\
1.30 \\
1.35 \\
1.40 \\
1.45 \\
1.50 \\
0.80 \\
0.85\end{array}$ & $\begin{array}{l}0.55695 \\
0.60150 \\
0.64585 \\
0.69001 \\
0.73401 \\
0.77784 \\
0.82152 \\
0.86506 \\
0.90847 \\
0.95176 \\
0.99493 \\
1.03800 \\
1.08096 \\
1.12382 \\
1.16660 \\
0.67599 \\
0.72311 \\
0.77012 \\
0.81705 \\
0.86389 \\
0.91066 \\
0.95734 \\
1.00396 \\
1.05051 \\
1.09699 \\
1.14342 \\
1.18980 \\
1.23612 \\
1.28240 \\
1.32863 \\
0.71574\end{array}$ & $\begin{array}{l}0.54485 \\
0.58912 \\
0.63318 \\
0.67705 \\
0.72074 \\
0.76426 \\
0.80762 \\
0.85084 \\
0.89391 \\
0.93686 \\
0.97968 \\
1.02239 \\
1.06500 \\
1.10750 \\
1.14991 \\
0.66992 \\
0.71690 \\
0.76377 \\
0.81055 \\
0.85724 \\
0.90384 \\
0.95036 \\
0.99681 \\
1.04319 \\
1.08951 \\
1.13576 \\
1.18196 \\
1.22810 \\
1.27420 \\
1.32024 \\
0.71168 \\
0.75956 \\
0.80738 \\
0.85514 \\
0.90283 \\
0.95047 \\
0.99805 \\
1.04559 \\
1.09308 \\
1.14053 \\
1.18793 \\
1.23530 \\
1.28264 \\
1.32994 \\
1.37720 \\
0.73261 \\
0.78096\end{array}$ & $\begin{array}{l}1.04305 \\
1.09850 \\
1.15415 \\
1.20999 \\
1.26599 \\
1.32216 \\
1.37848 \\
1.43494 \\
1.49153 \\
1.54824 \\
1.60507 \\
1.66200 \\
1.71904 \\
1.77618 \\
1.83340 \\
0.92401 \\
0.97689 \\
1.02988 \\
1.08295 \\
1.13611 \\
1.18934 \\
1.24266 \\
1.29604 \\
1.34949 \\
1.40301 \\
1.45658 \\
1.51020 \\
1.56388 \\
1.61760 \\
1.67137 \\
0.88426 \\
0.93628 \\
0.98837 \\
1.04051 \\
1.09271 \\
1.14497 \\
1.19927 \\
1.24963 \\
1.30202 \\
1.35446 \\
1.40693 \\
1.45945 \\
1.51199 \\
1.56457 \\
1.61717 \\
0.86434 \\
0.91592\end{array}$ & $\begin{array}{l}1.05515 \\
1.11088 \\
1.16682 \\
1.22295 \\
1.27926 \\
1.33574 \\
1.39238 \\
1.44916 \\
1.50609 \\
1.56314 \\
1.62032 \\
1.67761 \\
1.73500 \\
1.79250 \\
1.85009 \\
0.93008 \\
0.98310 \\
1.03623 \\
1.08945 \\
1.14276 \\
1.19616 \\
1.24964 \\
1.30319 \\
1.35681 \\
1.41049 \\
1.46424 \\
1.51804 \\
1.57190 \\
1.62580 \\
1.67976 \\
0.88832 \\
0.94044 \\
0.99262 \\
1.04486 \\
1.09717 \\
1.14953 \\
1.20195 \\
1.25441 \\
1.30692 \\
1.35947 \\
1.41207 \\
1.46470 \\
1.51736 \\
1.57006 \\
1.62280 \\
0.86739 \\
0.91904\end{array}$ & $\begin{array}{l}0.72908 \\
0.77457 \\
0.82005 \\
0.86552 \\
0.91099 \\
0.95645 \\
1.00189 \\
1.04733 \\
1.09276 \\
1.13818 \\
1.18359 \\
1.22899 \\
1.27439 \\
1.31977 \\
1.36514 \\
0.72939 \\
0.77482 \\
0.82024 \\
0.86564 \\
0.91102 \\
0.95638 \\
1.00173 \\
1.04706 \\
1.09237 \\
1.13766 \\
1.18293 \\
1.22819 \\
1.27343 \\
1.31865 \\
1.36385 \\
0.72970 \\
0.77508 \\
0.82043 \\
0.86576 \\
0.91105 \\
0.95632 \\
1.00157 \\
1.04678 \\
1.09197 \\
1.13714 \\
1.18227 \\
1.22738 \\
1.27246 \\
1.31752 \\
1.36254 \\
0.73002 \\
0.77534\end{array}$ & $\begin{array}{l}14.8946 \\
14.3217 \\
13.8232 \\
13.3869 \\
13.0027 \\
12.6627 \\
12.3604 \\
12.0905 \\
11.8485 \\
11.6308 \\
11.4342 \\
11.2561 \\
11.0944 \\
10.9470 \\
10.8124 \\
7.4636 \\
7.1773 \\
6.9282 \\
6.7102 \\
6.5182 \\
6.3483 \\
6.1972 \\
6.0623 \\
5.9414 \\
5.8326 \\
5.7343 \\
5.6453 \\
5.5644 \\
5.4908 \\
5.4235 \\
4.9879 \\
4.7972 \\
4.6313 \\
4.4860 \\
4.3581 \\
4.2449 \\
4.1443 \\
4.0544 \\
3.9738 \\
3.9013 \\
3.8358 \\
3.7765 \\
3.7226 \\
3.6735 \\
3.6286 \\
3.7508 \\
3.6079\end{array}$ & $\begin{array}{l}15.6496 \\
15.0484 \\
14.5254 \\
14.0675 \\
13.6644 \\
13.3077 \\
12.9906 \\
12.7075 \\
12.4536 \\
12.2252 \\
12.0191 \\
11.8323 \\
11.6626 \\
11.5081 \\
11.3669 \\
7.8416 \\
7.5412 \\
7.2798 \\
7.0510 \\
6.8495 \\
6.6713 \\
6.5128 \\
6.3713 \\
6.2444 \\
6.1302 \\
6.0272 \\
5.9338 \\
5.8490 \\
5.7717 \\
5.7011 \\
5.2401 \\
5.0400 \\
4.8659 \\
4.7134 \\
4.5792 \\
4.4605 \\
4.3549 \\
4.2606 \\
4.1760 \\
4.1000 \\
4.0313 \\
3.9691 \\
3.9125 \\
3.8610 \\
3.8139 \\
3.9402 \\
3.7902\end{array}$ \\
\hline
\end{tabular}


WSRC-TR-92-259

Task 92-053-1

May 29, 1992

Revision 0

Statistlcal Analysis of Test Data for APM Rod Issue Page 51 of 87

TABIR 17

$(A C-A P B 8)$

( CONTINOED )

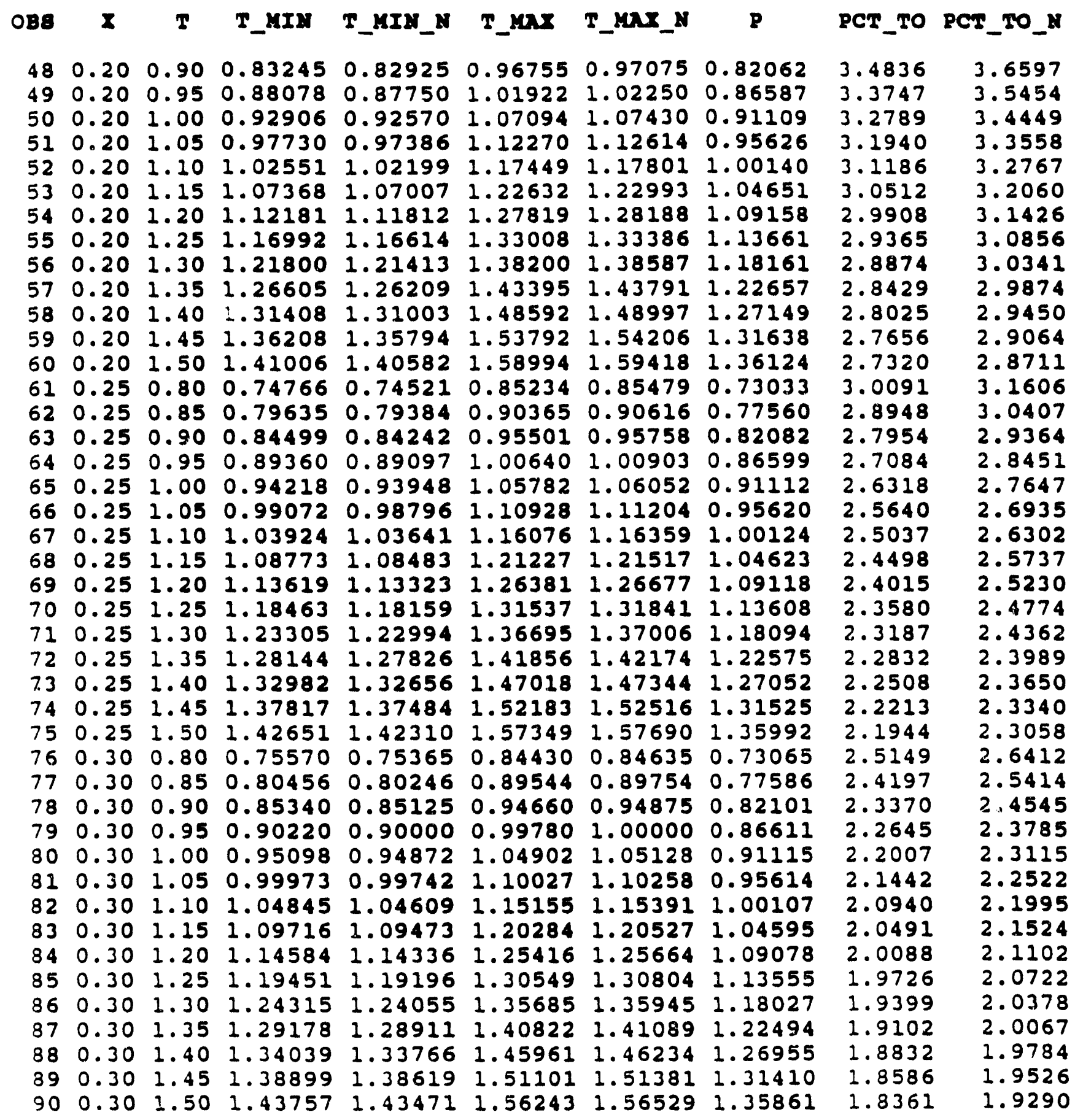


WSRC-TR-92-259

Task 92-053-1

Statistlcal Analysis of Test Data for APM Rod Issue
May 29, 1992

Revision 0

\section{TABIF 18}

$(\mathrm{AC}-\mathrm{APY} 9)$

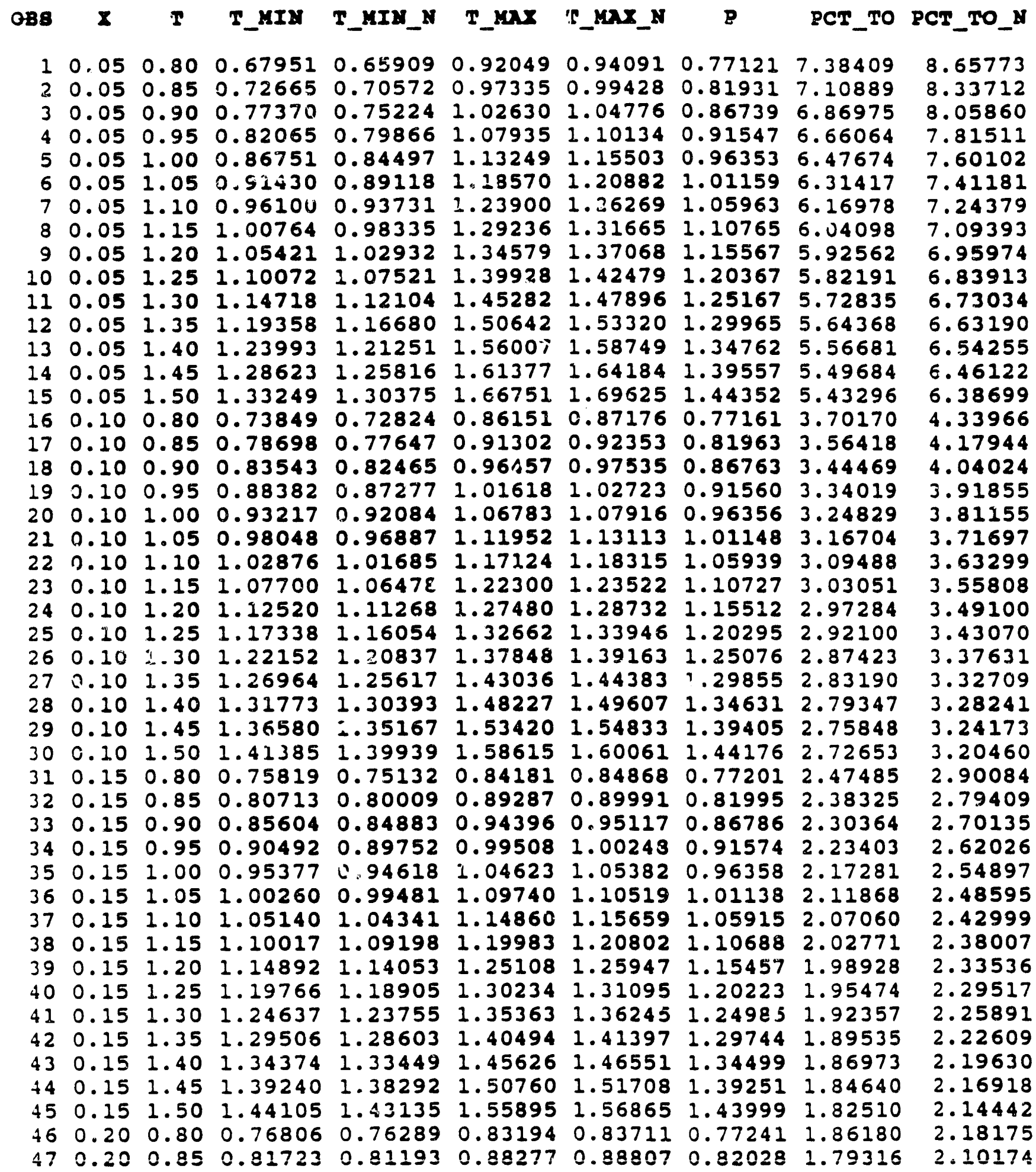


WSRC-TR-92-259

Task 92-053-1

Statlstical Analysis of Test Data for APM Rod Issue

May 29, 1992

Revision 0

TA3LR 18

$($ AC - APH 9$)$

( CONTINOED )

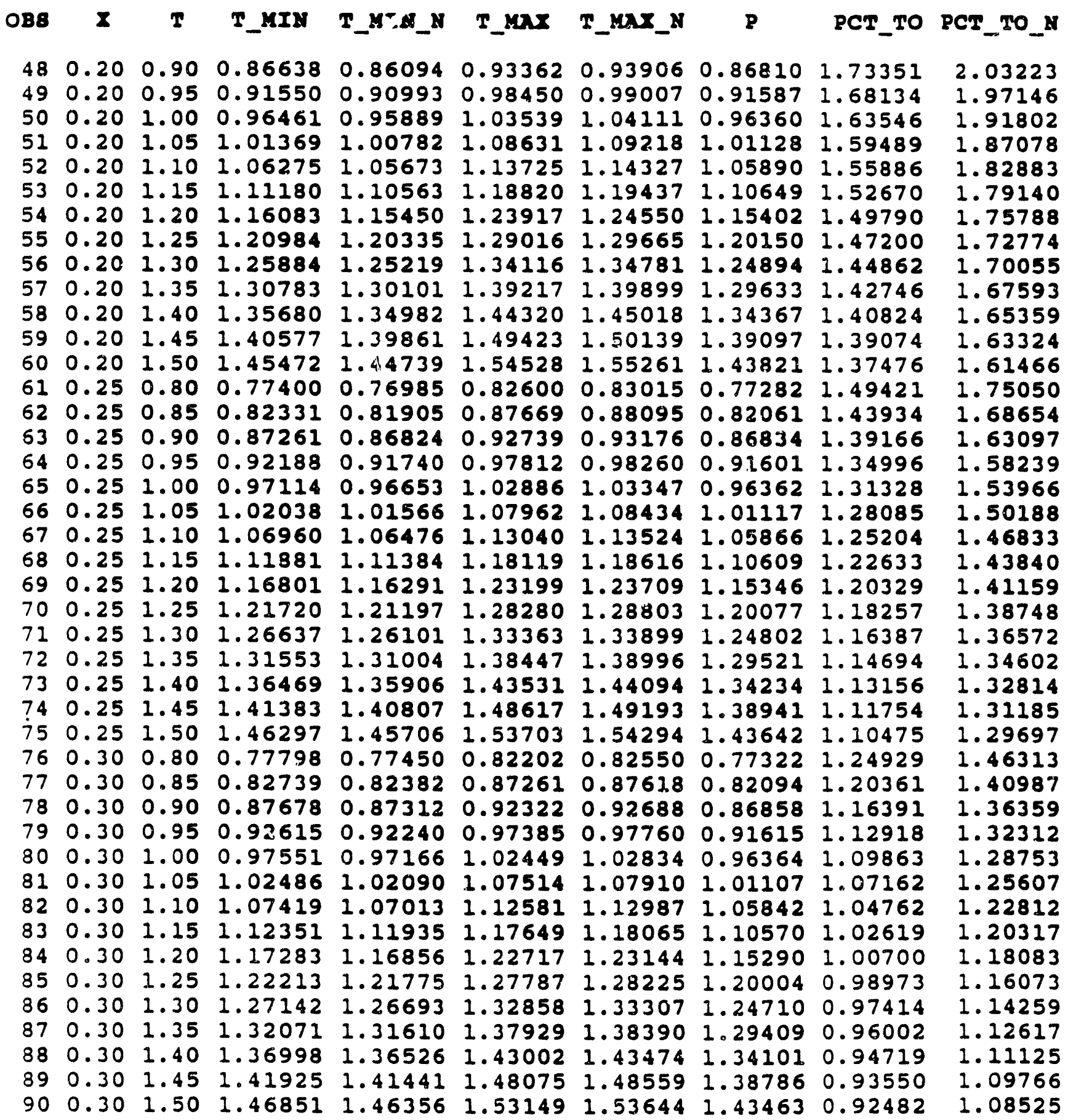


WSRC-TR-92-259

Task 92-053-1

May 29, 1992

Revision 0

Starlstlcal Analysis of Test Data for APM Rod Issue Page 54 of 87

11.0 Appendices

\begin{abstract}
APPENDIX 1: DC - APY 1, DC - APY 2 DATA
APPEXIII 2: DC - APM 3, DC - APM 4 DATA

APPEEDIX 3: DC - APY 3, DC - APY 6 DATA

APPENDIX 4: DC - APH 7, DC - APK 8 DATA

APPENDIX 5: DC - APY 9 DATA

APPEADIX 6: AC - APH 1, AC - APH 2 DATA

APPESDIX 7: AC - APY 3, AC - APX 4 DATA

APPEADIX 8: AC - APH 5, AC - APY 6 DATA

APPENDIX 9: AC - APM 7, AC - APY 8 DATA

APPENDIX 10: AC - APH O DATA
\end{abstract}

$$
\begin{aligned}
& X=\text { POWBR IX WATS/GRAY } \\
& Y 2=\text { 8EM8OR } 2 \text { (MV) } \\
& Y 6=\text { 8ENBOR } 6 \text { (MV) }
\end{aligned}
$$

APPENDIX 11: DC REGRES8ION 8TATISTICS FROK SA8 PROGRAM APPENDIX 12: AC REGREBBION BTATISTIC8 FROY SAS PROGRAM APPEADIX 13: SAB PROGRNA FOR APA CALIBRATION APPEADIX 14: METHOD OF PROPACATION OY ERRORS 
DC - APY 1

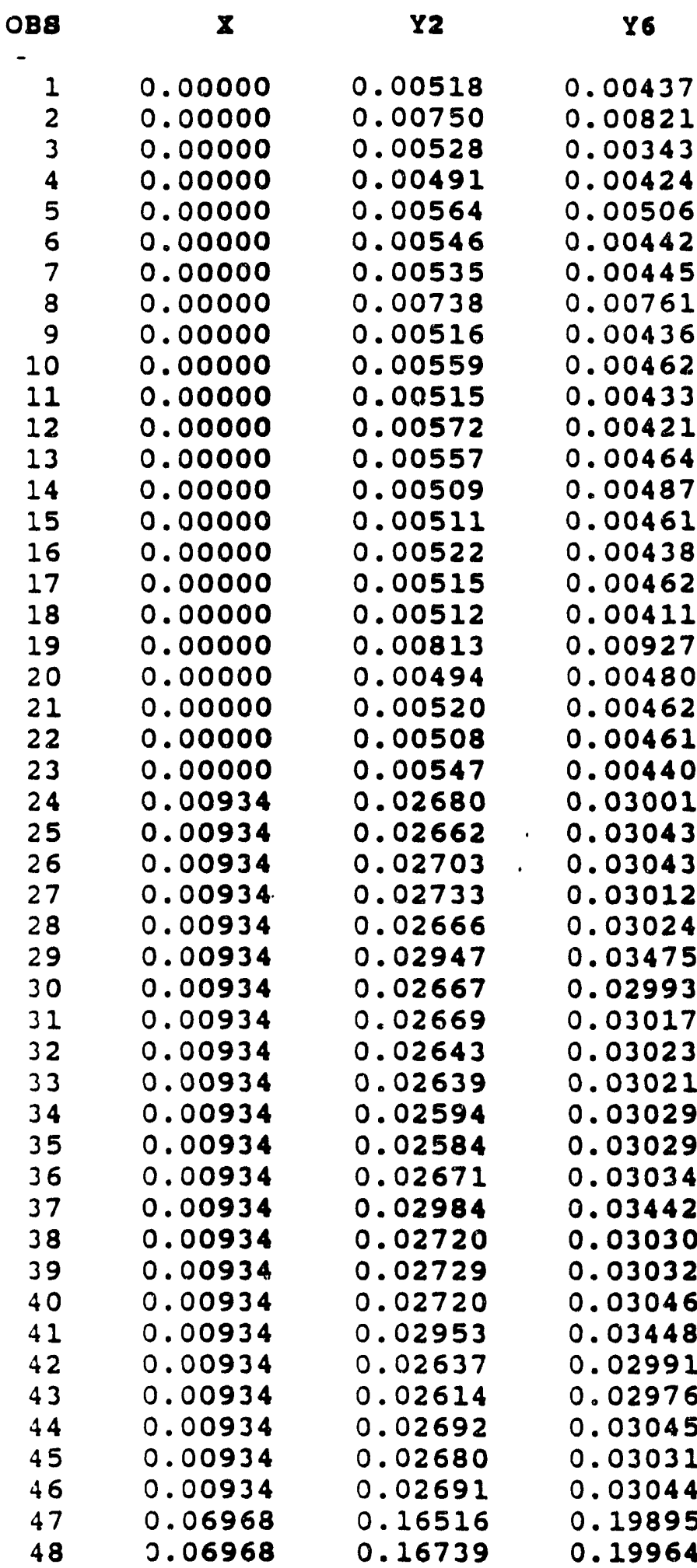

DC - APM 2

Y2

Y 6

0.07366

0.07366

0.07366

0.07366

0.07366

0.07366

0.07366

0.07366

0.07366

0.07366

0.07366

0.07366

0.07366

0.07366

0.07366

0.07366

0.07366

0.07366

0.07366

0.07366

0.07366

0.07366

0.07366

0.07366

0.07366

0.07366

0.00934

0.00934

0.00934

0.00934

0.00934

0.00934

0.00934

0.00934

0.00934

0.00934

0.00934

0.00934

0.00934

0.00934

0.00934

0.00934

0.00934

0.00934

0.00934

0.00934

0.00934

0.00934
0.18134

0.18115

0.18195

0.18164

0.18062

0.18030

0.18063

0.17966

0.17759

0.17778

0.17917

0.18027

0.18106

0.18136

0.18215

0.18091

0.18044

0.18067

0.17976

0.18009

0.18221

0.18238

0.18227

0.18277

0.18152

0.18170

0.02623

0.02644

0.02580

0.02578

0.02637

0.02626

0.02630

0.02590

0.02601

0.02642

0.02614

0.02571

0.02583

0.02668

0.02644

0.02618

0.02608

0.02643

0.02638

0.02663

0.02612

0.02669
0.18812

0.18873

0.18914

0.18833

0.18801

0.18838

0.18835

0.18889

0.18844

0.18845

0.18876

0.18851

0.18900

0.18804

0.18813

0.18829

0.18810

0.18871

0.18929

0.18971

0.18937

0.18896

0.18876

0.18845

0.18805

0.18893

0.02723

0.02718

0.02728

0.02666

0.02749

0.02771

0.02767

0.02626

0.02759

0.02810

0.02798

0.02666

0.02724

0.02760

0.02787

0.02731

0.02743

0.02741

0.02788

0.02799

0.02711

0.02829 
WSRC-TR-92-259

Task 92-053-1

May 29, 1992

Revision 0

Statistical Analysis of Test Data for APM Rod Issue Page 56 of 87

\section{APPENDIX 1}

( CONTINOED)

DC - APY 1

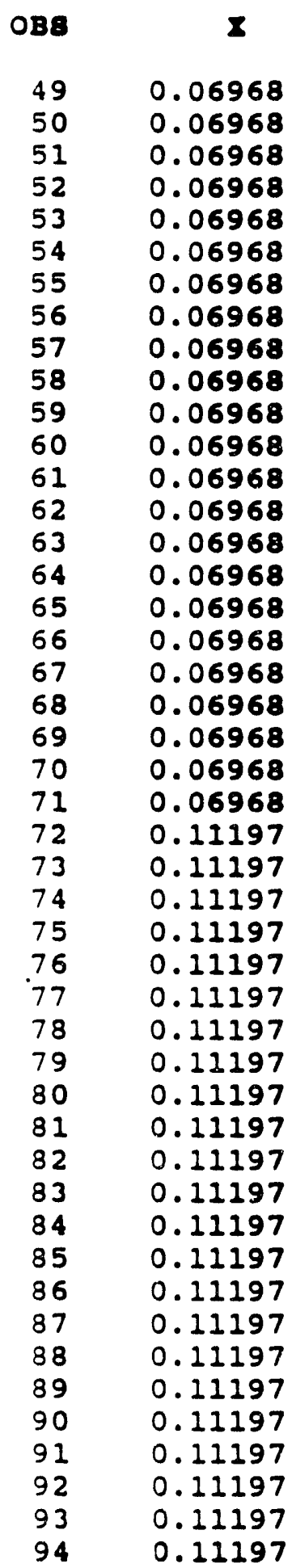

0.16681

0.16789
$\mathbf{Y 2}$

16

0.17005

0.16822

0.16622

0.16606

0.16680

0.16917

0.17125

0.17339

0.17209

0.16940

0.16960

0.17057

0.16795

0.16488

0.16877

0.16869

0.16757

0.16825

0.17144

0.16930

0.26712

0.27172

0.27003

0.26954

0.27123

0.27225

0.26996

0.26874

0.26994

0.27045

0.26740

0.26902

0.26603

0.26605

0.26632

0.27171

0.26808

0.26326

0.26158

0.26739

0.26658

0.26647

0.26649
0.19966

0.20245

0.19910

0.19878

0.19953

0.19960

0.19944

0.19904

0.19919

0.20402

0.19930

0.19861

0.19918

0.20236

0.19902

0.19971

0.19952

0.20347

0.19929

0.19963

0.19978

0.20435

0.19901

0.31725

0.32224

0.31725

0.31753

0.31765

0.31695

0.31696

0.31657

0.31701

0.31631

0.31647

0.32007

0.31679

0.31711

0.31771

0.32094

0.31673

0.31614

0.31652

0.32057

0.31655

0.31617

0.31631
$\Sigma$

0.00934

0.00934

0.00934

0.00934

0.06968

0.06968

0.06968

0.06968

0.06968

0.06968

0.06968

0.06968

0.06968

0.06968

0.06968

0.06968

0.06968

0.06968

0.06968

0.06968

0.06968

0.06968

0.06968

0.06968

0.06968

0.06968

0.06968

0.06968

0.06968

0.10949

0.10949

0.10949

0.10949

0.10949

0.10949

0.10949

0.10949

0.10949

0.10949

0.10949

0.10949

0.10949

0.10949

0.10949

0.10949

0.10949
DC - APM 2

$Y 2$

16

0.02621

0.02688

0.02633

0.02583

0.17174

0.17201

0.17210

0.17204

0.17066

0.17031

0.17277

0.17367

0.17411

0.17388

0.17203

0.17253

0.17241

0.17259

0.17256

0.17156

0.17118

0.17231

0.17132

0.17258

0.17313

0.17169

0.17255

0.17419

0.17419

0.26712

0.26518

0.26435

0.26559

0.26771

0.26655

0.26811

0.26708

0.26969

0.27065

0.27143

0.27000

0.26755

0.26580

0.26589

0.26422

0.26624
0.02766

0.02800

0.02771

0.02665

0.17974

0.17983

0.17977

0.18017

0.18037

0.18038

0.18063

0.18043

0.18041

0.18071

0.17994

0.18023

0.18008

0.18042

0.18039

0.17977

0.18079

0.18061

0.18033

0.18024

0.18072

0.18048

0.18038

0.18055

0.18077

0.27974

0.27826

0.27871

0.27867

0.27944

0.27967

0.27914

0.27924

0.28029

0.28016

0.27824

0.27774

0.27771

0.27772

0.27838

0.27866

0.27966 
WSRC-TR-92-259

Task 92-053-1

May 29, 1992

Statistical Analysis of Test Data for APM Rod Issue Revision 0

Page 57 of 87

\section{APPENDIX 1}

( CONTINOED )

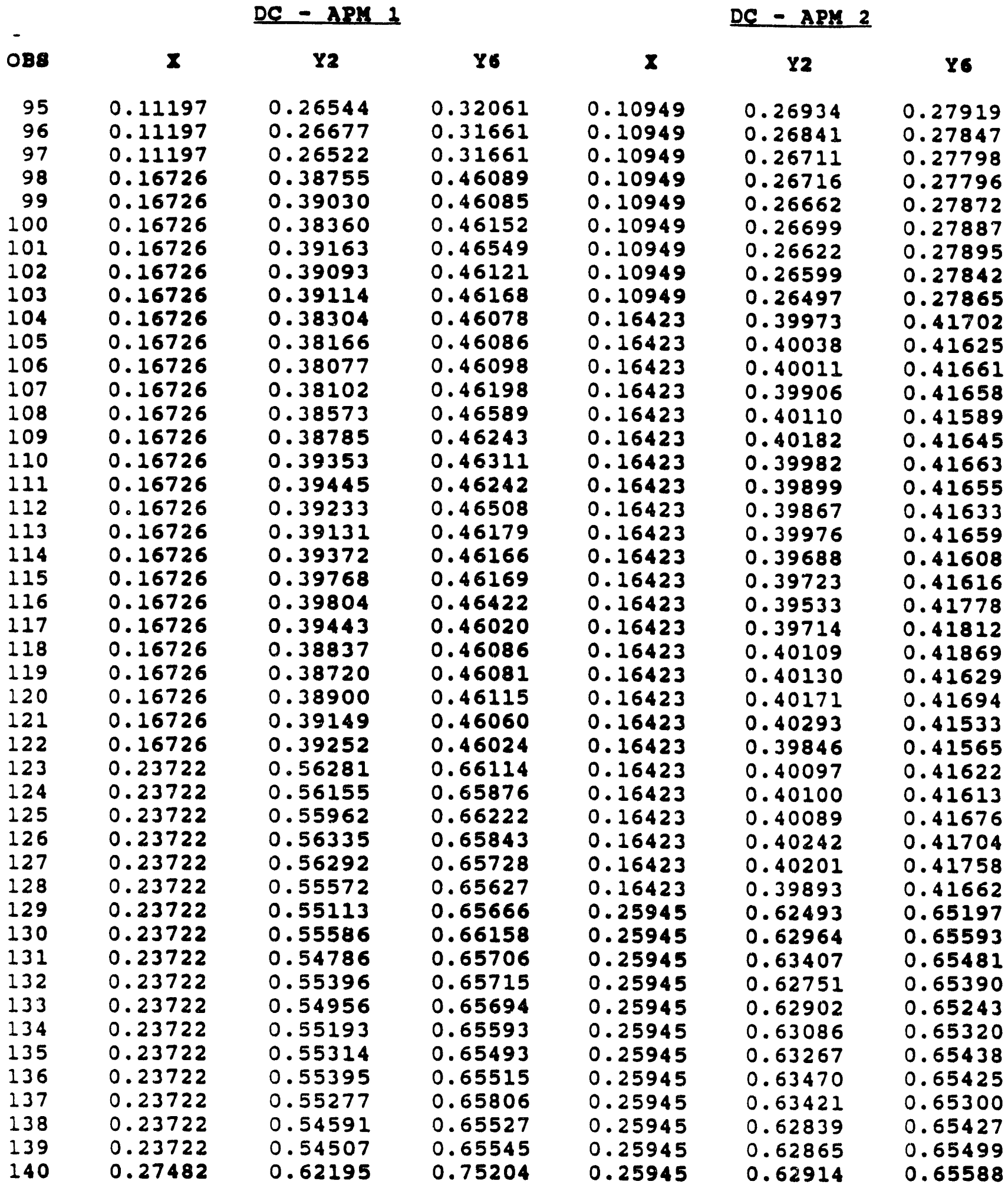




\section{APPENDIX 1}

( CONTINOED )

088

$\mathbf{x}$

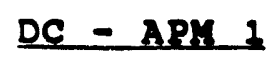

$\begin{array}{lll}141 & 0.27482 & 0.63655 \\ 142 & 0.27482 & 0.63088 \\ 143 & 0.27482 & 0.65040 \\ 144 & 0.27482 & 0.63486 \\ 145 & 0.27482 & 0.62379 \\ 146 & 0.27482 & 0.62363 \\ 147 & 0.27482 & 0.63292 \\ 148 & 0.27482 & 0.63724 \\ 149 & 0.27482 & 0.63851 \\ 150 & 0.27482 & 0.62557 \\ 151 & 0.27482 & 0.62720 \\ 152 & 0.27482 & 0.62628 \\ 153 & 0.27482 & 0.62885 \\ 154 & 0.27482 & 0.62784 \\ 155 & 0.27482 & 0.63936 \\ 156 & 0.27482 & 0.64037 \\ 157 & 0.27482 & 0.63778 \\ 158 & 0.27482 & 0.64120 \\ 159 & 0.27482 & 0.63458 \\ 160 & 0.27482 & 0.63127 \\ 161 & 0.27482 & 0.63753 \\ 162 & 0.27482 & 0.63289 \\ 163 & 0.27482 & 0.64038 \\ 164 & 0.27482 & 0.62920 \\ 165 & 0.31102 & 0.72221 \\ 166 & 0.31102 & 0.73521 \\ 167 & 0.31102 & 0.72090 \\ 168 & 0.31102 & 0.72548 \\ 169 & 0.31102 & 0.72143 \\ 170 & 0.31102 & 0.72243 \\ 171 & 0.31102 & 0.72179 \\ 172 & 0.31102 & 0.71646 \\ 173 & 0.31102 & 0.71203 \\ 174 & 0.31102 & 0.71068 \\ 175 & 0.31102 & 0.71572 \\ 176 & 0.31102 & 0.72044 \\ 177 & 0.31102 & 0.72231 \\ 178 & 0.31102 & 0.72383 \\ 179 & 0.31102 & 0.72301 \\ 180 & 0.31102 & 0.72362 \\ 181 & 0.31102 & 0.71996 \\ 182 & 0.31102 & 0.72330 \\ 183 & 0.31102 & 0.72790 \\ 184 & 0.31102 & 0.73474 \\ 185 & 0.31102 & 0.72768 \\ 186 & 0.31102 & 0.71780\end{array}$

Y6

\begin{abstract}
0.75212
\end{abstract}
0.75225

0.75135

0.74992

0.74856

0.75045

0.75351

0.75042

0.74976

0.75137

0.75625

0.75248

0.75258

0.75230

0.75195

0.75243

0.75163

0.75151

0.75195

0.75200

0.75243

0.75182

0.75192

0.75179

0.85372

0.85380

0.85375

0.85704

0.85275

0.85121

0.85067

0.85068

0.85079

0.85230

0.85631

0.85327

0.85291

0.85429

0.85320

0.85522

0.85185

0.85307

0.85278

0.85452

0.85105

0.85090

DC - APY 2

z $Y 2$

Y6

\subsection{5}

0.25945

0.25945

0.25945

0.25945

0.25945

0.25945

0.25945

0.25945

0.25945

0.25945

0.25945

0.25945

0.25945

0.25945

0.27093

0.27093

0.27093

0.27093

0.27093

0.27093

0.27093

0.27093

0.27093

0.27093

0.27093

0.27093

0.27093

0.27093

0.27093

0.27093

0.27093

0.27093

0.27093

0.27093

0.27093

0.27093

0.27093

0.27093

0.27093

0.27093

0.31102

0.31102

0.31102

0.31102

0.31102
0.63370

0.63285

0.63495

0.63536

0.63739

0.63209

0.62939

0.62550

0.62634

0.62663

0.63041

0.63057

0.63280

0.62954

0.62711

0.64740

0.65133

0.66364

0.66296

0.65835

0.65919

0.66075

0.65865

0.65870

0.65639

0.65802

0.65687

0.65700

0.65905

0.65819

0.65703

0.65578

0.65695

0.65904

0.65680

0.65744

0.65803

0.65791

0.65677

0.65731

0.65841

0.74594

0.75038

0.74760

0.74665

0.74119
0.65835

0.65625

0.65539

0.65256

0.65219

0.65101

0.65124

0.65055

0.65229

0.65225

0.65500

0.65386

0.65186

0.65077

0.65151

0.68480

0.68415

0.68153

0.68078

0.68107

0.68083

0.68222

0.68318

0.68241

0.68212

0.68323

0.68250

0.68046

0.67978

0.68137

0.68178

0.68210

0.68341

0.68305

0.68371

0.68440

0.68155

0.67992

0.68215

0.68222

0.68103

0.77397

0.77498

0.77038

0.77152

0.77370 


\section{APPENDIF 1 \\ ( CONTINOED )}

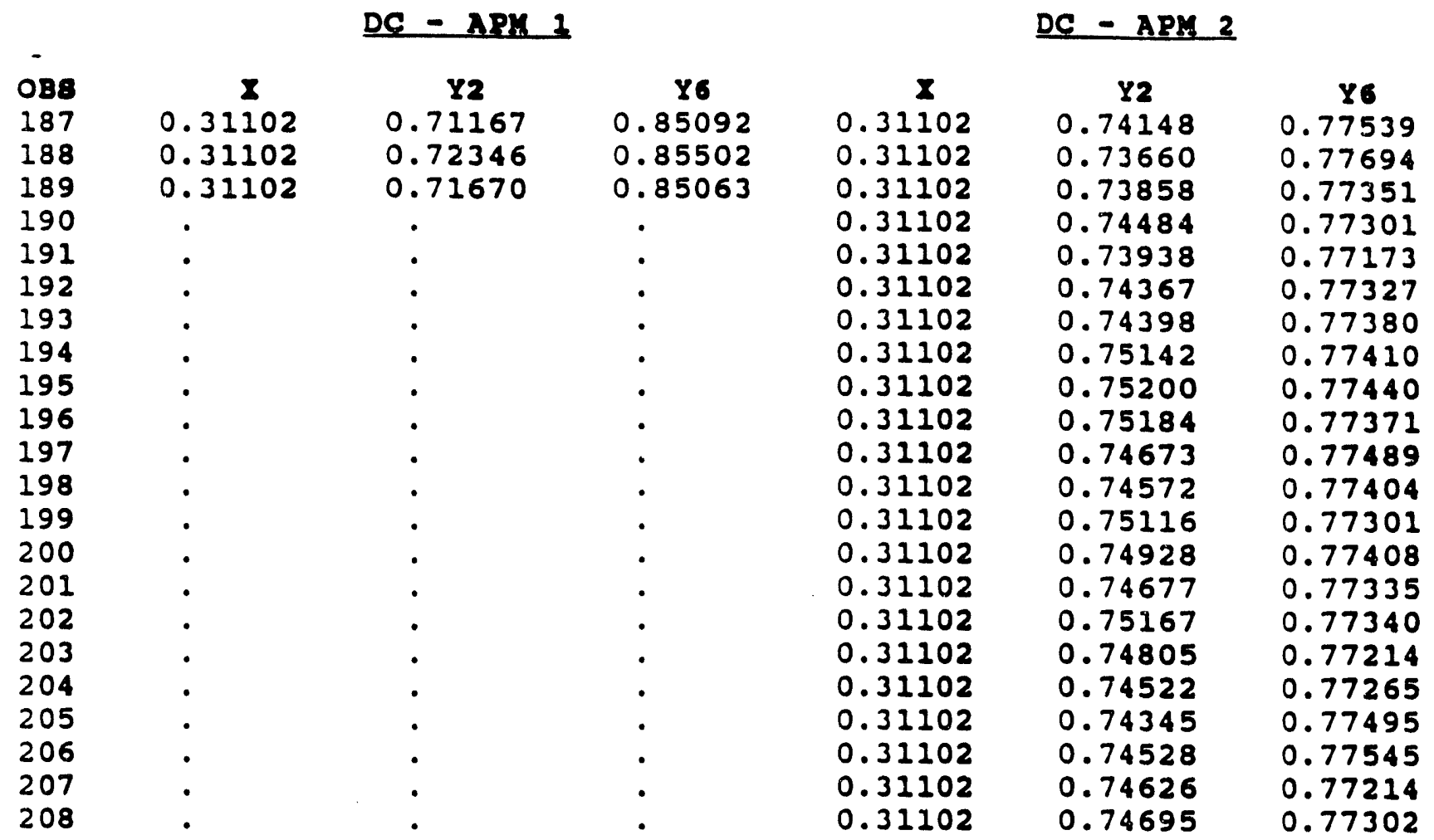


WSRC-TR-92-259

Task 92-053-1

May 29, 1992

Revision 0

Statistlcal Analysis of Test Data for APM Rod Issue Page 60 of 87

APPENDIX 2

DC - APY 3

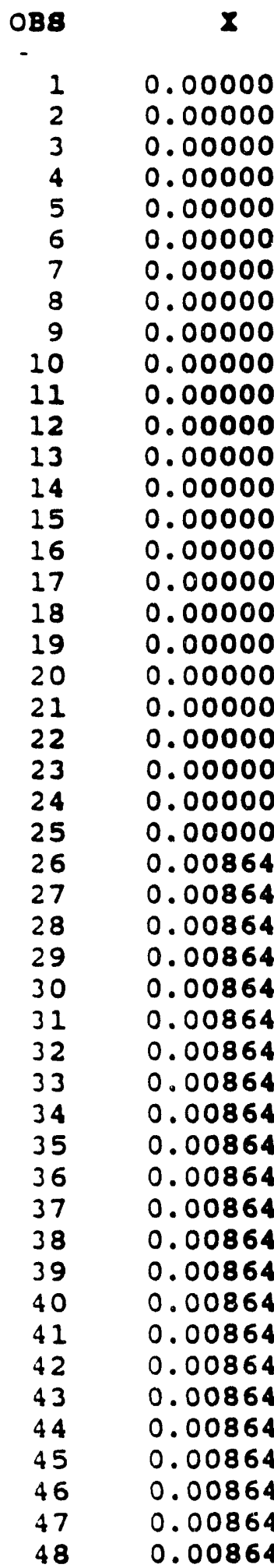

$Y 2$

0.00347

0.00450

0.00414

0.00394

0.00402

0.00486

0.00416

0.00371

0.00388

0.00404

0.00388

0.00424

0.00353

0.00380

0.00387

0.00421

0.00397

0.00438

0.00442

0.00419

0.00398

0.00410

0.00367

0.00404

0.00372

0.02553

0.02593

0.02506

0.02469

0.02495

0.02563

0.02511

0.02527

0.02494

0.02514

0.02517

0.02507

0.02512

0.02526

0.02560

0.02550

0.02548

0.02555

0.02538

0.02565

0.02533

0.02549

0.02554
Y6

8

0.00442

0.00396

0.00401

0.00404

0.00377

0.00488

0.00394

0.00352

0.00364

0.00414

0.00331

0.00399

0.00391

0.00370

0.00350

0.00418

0.00394

0.00416

0.00404

0.00381

0.00318

0.00376

0.00303

0.00367

0.00332

0.02772

0.02786

0.02748

0.02763

0.02747

0.02787

0.02752

0.02788

0.02750

0.02784

0.02769

0.02750

0.02756

0.02768

0.02777

0.02753

0.02815

0.02807

0.02767

0.02786

0.02696

0.02783

0.02730

DC - APY 4

$Y 2$

$Y 6$

0.00000

0.00000

0.00000

0.00000

0.00000

0.00000

0.00000

0.00000

0.00000

0.00000

0.00000

0.00000

0.00000

0.00000

0.00000

0.00000

0.00000

0.00000

0.00000

0.00000

0.00000

0.00000

0.00000

0.00000

0.00000

0.00000

0.00000

0.00864

0.00864

0.00864

0.00864

0.00864

0.00864

0.00864

0.00864

0.00864

0.00864

0.00864

0.00864

0.00864

0.00864

0.00864

0.00864

0.00864

0.00864

0.00864

0.00864

0.00864
0.00423

0.00470

0.00437

0.00434

0.00451

0.00438

0.00447

0.00479

0.00455

0.00452

0.00422

0.00417

0.00391

0.00410

0.00475

0.00455

0.00481

0.00425

0.00380

0.00453

0.00414

0.00417

0.00428

0.00490

0.00464

0.00419

0.00446

0.02485

0.02438

0.02460

0.02442

0.02497

0.02476

0.02456

0.02490

0.02444

0.02498

0.02477

0.02438

0.02563

0.02483

0.02470

0.02484

0.02474

0.02474

0.02397

0.02436

0.02467
0.00546

0.00510

0.00493

0.00546

0.00506

0.00531

0.00473

0.00505

0.00509

0.00492

0.00484

0.00455

0.00488

0.00507

0.00491

0.00530

0.00489

0.00473

0.00512

0.00503

0.00551

0.00488

0.00501

0.00530

0.00502

$0.0 J 529$

0.00510

0.02887

0.02875

r. 02875

?. 02878

0.02365

0.02891

0.02915

0.02889

0.02935

0.02906

0.02861

0.02873

0.02890

0.02930

0.02897

0.02918

0.02890

0.02907

0.02871

0.02906

0.02920 
WSRC-TR-92-259

Task 92-053-1

May 29, 1992

Statistical Analvsis of Test Data for APM Rod Issue Revision 0

Page 61 of 87

\section{APPENDIX 2}

( CONTINOED )

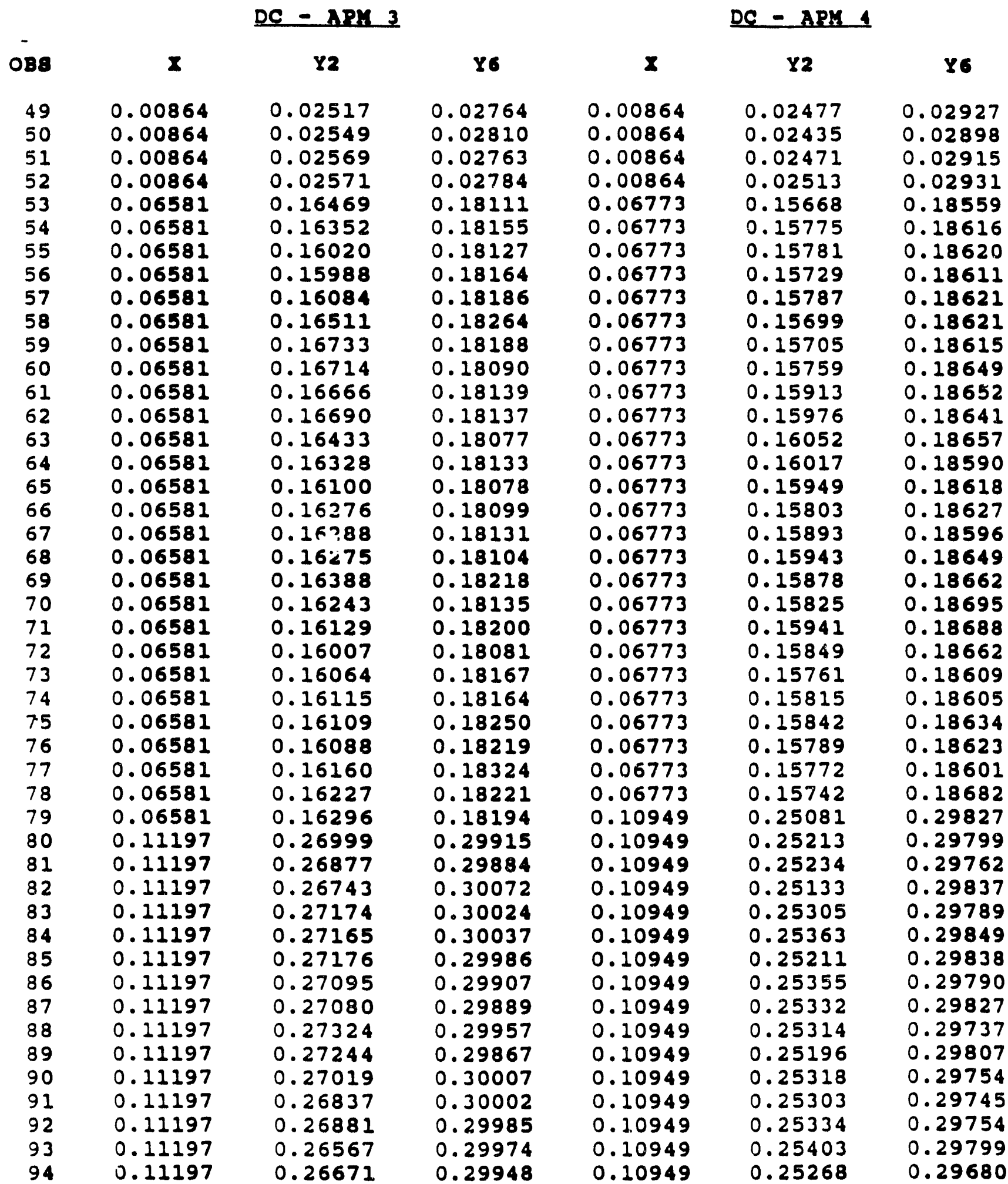


WSRC-TR-92-259

Task 92-053-1

May 29, 1992

Revision 0

Statlstleal Analysis of Test Data for APM Rod issue Page 62 of 87

\author{
APPENDIX 2
}

( CONTINOED )

$D C-A P Y 3$

OB8

$\begin{array}{ll}95 & 0.11197 \\ 96 & 0.11197 \\ 97 & 0.11197 \\ 98 & 0.11197 \\ 99 & 0.11197 \\ 100 & 0.11197 \\ 101 & 0.11197 \\ 102 & 0.11197 \\ 103 & 0.11197 \\ 104 & 0.11197 \\ 105 & 0.16726 \\ 106 & 0.16726 \\ 107 & 0.16726 \\ 108 & 0.16726 \\ 109 & 0.16726 \\ 110 & 0.16726 \\ 111 & 0.16726 \\ 112 & 0.16726 \\ 113 & 0.16726 \\ 114 & 0.16726 \\ 115 & 0.16726 \\ 116 & 0.16726 \\ 117 & 0.16726 \\ 118 & 0.16726 \\ 119 & 0.16726 \\ 120 & 0.16726 \\ 121 & 0.16726 \\ 122 & 0.16726 \\ 123 & 0.16726 \\ 124 & 0.16726 \\ 125 & 0.16726 \\ 126 & 0.16726 \\ 127 & 0.16726 \\ 128 & 0.16726 \\ 129 & 0.16726 \\ 130 & 0.23361 \\ 131 & 0.23361 \\ 132 & 0.23361 \\ 133 & 0.23361 \\ 134 & 0.23361 \\ 135 & 0.23361 \\ 136 & 0.23361 \\ 137 & 0.23361 \\ 138 & 0.23361 \\ 140 & 0.23361 \\ 139 & 0.23361 \\ 130\end{array}$

42

0.26892

0.26908

0.27058

0.26865

0.26803

0.26899

0.26866

0.27316

0.27850

0.27652

0.39708

0.39870

0.40693

0.40167

0.39949

0.39713

0.39335

0.39169

0.39232

0.39352

0.39852

0.39923

0.40100

0.40575

0.40475

0.39996

0.39968

0.40233

0.40125

0.40174

0.40140

0.40141

0.39615

0.40138

0.40727

0.54886

0.54729

0.54990

0.55455

0.55794

0.55881

0.55650

0.55946

0.56390

0.56326

0.56315
16

0.30049
0.29924
0.29976
0.29919
0.30030
0.30049
0.30119
0.30150
0.30057
0.30055

0.44191

0.44309

0.44262

0.44290

0.44259

0.44337

0.44301

0.44309

0.44417

0.44503

0.44442

0.44487

0.44443

0.44470

0.44387

0.44396

0.44418

0.44487

0.44414

0.44456

0.44484

0.44536

0.44434

0.44473

0.44444

0.61835

0.61947

0.61933

0.61903

0.62021

0.62104

0.62090

0.62053

0.62011

0.61932

0.61811

DC - APY 1

$\mathbf{Y 2}$

16

0.10949

0.10949

0.10949

0.10949

0.10949

0.10949

0.10949

0.10949

0.10949

0.16423

0.16423

0.16423

0.16423

0.16423

0.16423

0.16423

0.16423

0.16423

0.16423

0.16423

0.16423

0.16423

0.16423

0.16423

0.16423

0.16423

0.16423

0.16423

0.16423

0.16423

0.16423

0.16423

0.16423

0.16423

0.16423

0.16423

0.23003

0.23003

0.23003

0.23003

0.23003

0.23003

0.23003

0.23003

0.23003

\subsection{7}

0.25267

0.25072

0.25069

0.25173

0.25283

0.25216

0.25577

0.25569

0.25374

0.37860

0.38015

0.37951

0.38136

0.38130

0.37985

0. 38083

0.38311

0.38083

0.38227

0.38105

0.37962

0.37781

0.37586

0.37839

0.37953

0.38230

0.38310

0.38388

0.38253

0.38165

0.38259

0.38141

0.38154

0.38084

0.37883

0.38228

0.52588

0.51979

0.52236

0.52786

0.52401

0.52334

0.52320

0.52242

0.52010
0.29699

0.29727

0.29706

0.29755

0.29812

0.29960

0.29828

0.29782

0.29764

0.29650

0.44607

0.44641

0.44717

0.44675

0.44583

0.44581

0.44723

0.44656

0.44641

0.44676

0.44641

0.44630

0.44653

0.44783

0.44801

0.44805

0.44866

0.44761

0.44842

0.44768

0.44780

0.44855

0.44802

0.44713

0.44725

0.44741

0.44849

0.61620

0.61658

0.61640

0.61649

0.61754

0.61643

0.61536

0.61617

0.61561 
WSRC.TR-92-259

Task 92-053-1

May 29, 1992

Statlstical Analysis of Test Data for APM Rod Issue Page 63 of 87

\section{APPENDIX 2}

( CORTINOED )

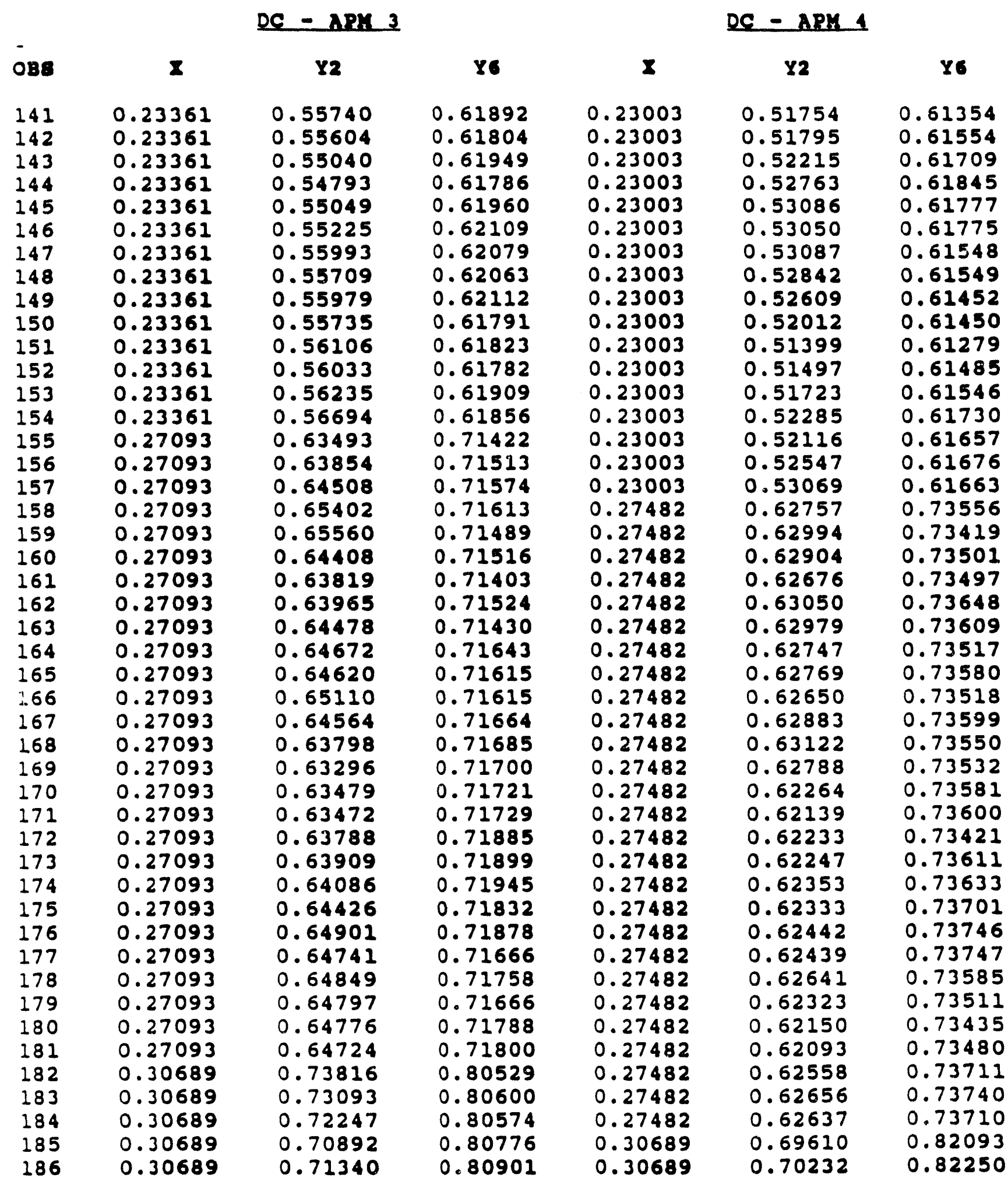


WSRC-TR-92-259

Task 92-053-1
jtatistical Analysis of Test Data for APM Rod Issue Page 64 of 87

May 29, 1992

\author{
APPENDIX 2 \\ ( CONTINOED )
}

DC - NPY 3

088

$\begin{array}{ll}187 & 0.30689 \\ 188 & 0.30689 \\ 189 & 0.30689 \\ 190 & 0.30689 \\ 191 & 0.30689 \\ 192 & 0.30689 \\ 193 & 0.30689 \\ 194 & 0.30689 \\ 195 & 0.30689 \\ 196 & 0.30689 \\ 197 & 0.30689 \\ 198 & 0.30689 \\ 199 & 0.30689 \\ 200 & 0.30689 \\ 201 & 0.30689 \\ 202 & 0.30689 \\ 203 & 0.30689 \\ 204 & 0.30689 \\ 205 & 0.30689 \\ 206 & 0.30689 \\ 207 & 0.30689 \\ 208 & 0.30689 \\ 209 & . \\ 210 & . \\ 211 & . \\ 212 & .\end{array}$

\begin{abstract}
$\mathbf{Y 2}$
\end{abstract}

$$
\begin{aligned}
& 0.72130 \\
& 0.74645 \\
& 0.74914 \\
& 0.74520 \\
& 0.74375 \\
& 0.73357 \\
& 0.74182 \\
& 0.75691 \\
& 0.75018 \\
& 0.73838 \\
& 0.72213 \\
& 0.71977 \\
& 0.72255 \\
& 0.73268 \\
& 0.73061 \\
& 0.71808 \\
& 0.72078 \\
& 0.72457 \\
& 0.72991 \\
& 0.73253 \\
& 0.72577 \\
& 0.71482
\end{aligned}
$$

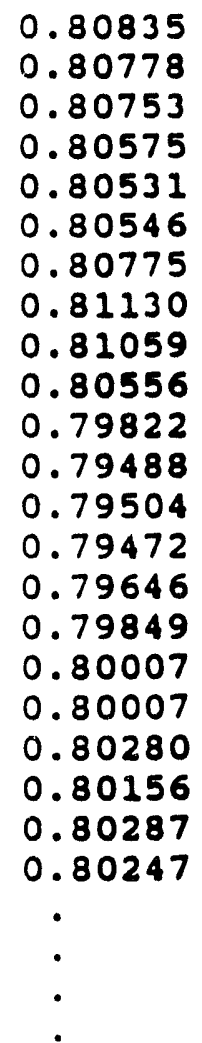

$D C-A P Y 4$

Y2 $\mathbf{Y 6}$
0.30689
0.30689
0.30689
0.30689
0.30689
0.30689
0.30689
0.30689
0.30689
0.30689
0.30689
0.30689
0.30689
0.30689
0.30689
0.30689
0.30689
0.30689
0.30689
0.30689
0.30689
0.30689
0.30689
0.30689
0.30689
0.30689

0.82383

0.82383

0.82362

0.82276

0.82410

0.82415

0.82277

0.82274

0.82236

0.82394

0.82403

0.82382

0.82261

0.82282

0.82284

0.82282

0.82193

0.82301

0.82280

0.82427

0.82451

0.82432

0.82455

0.82396

0.82526

0.82570 


\section{APPENDIX 3}

$\underline{D C}-A P X 5$

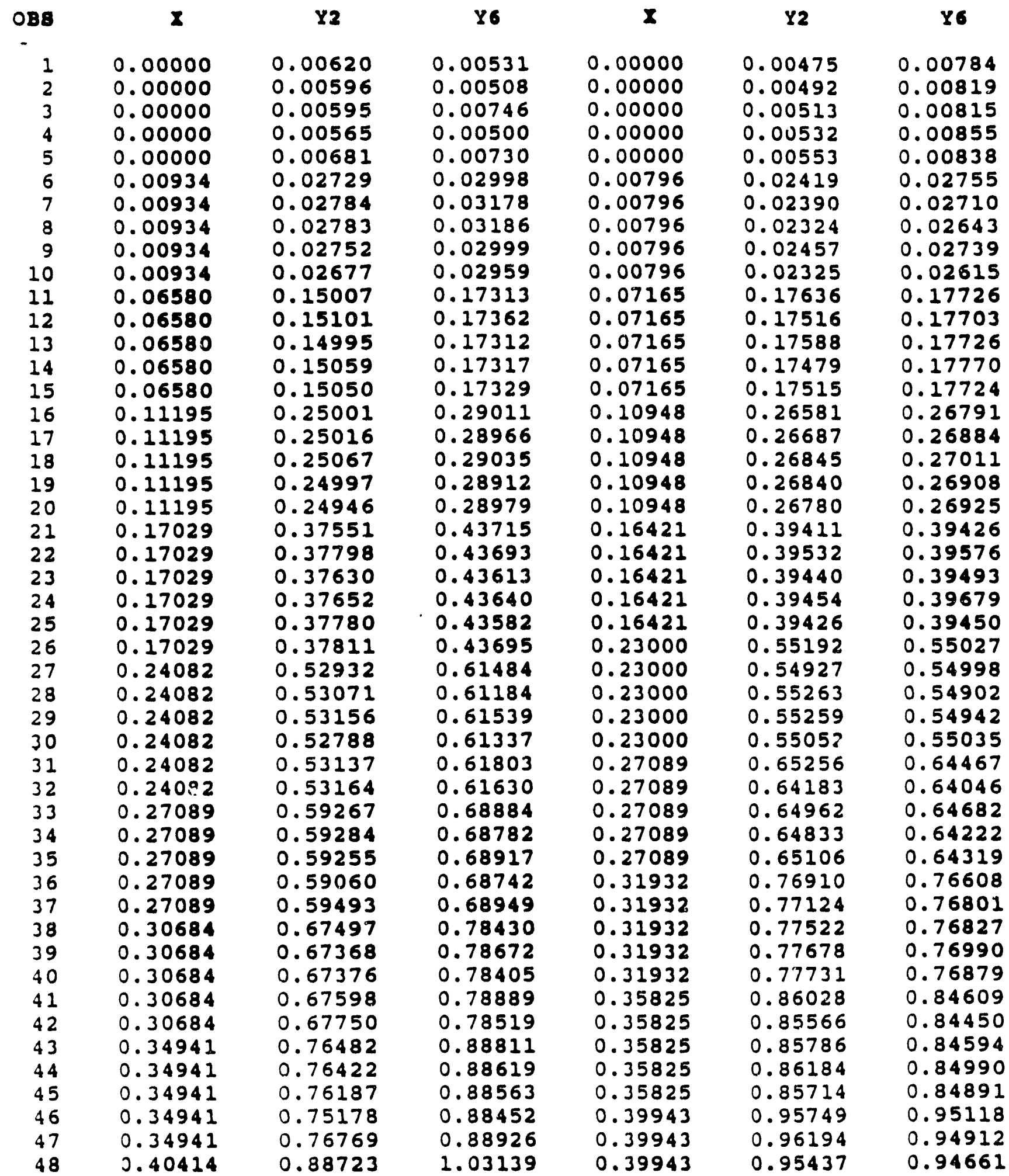




\author{
APPENDIX 3 \\ ( CONTINUED )
}

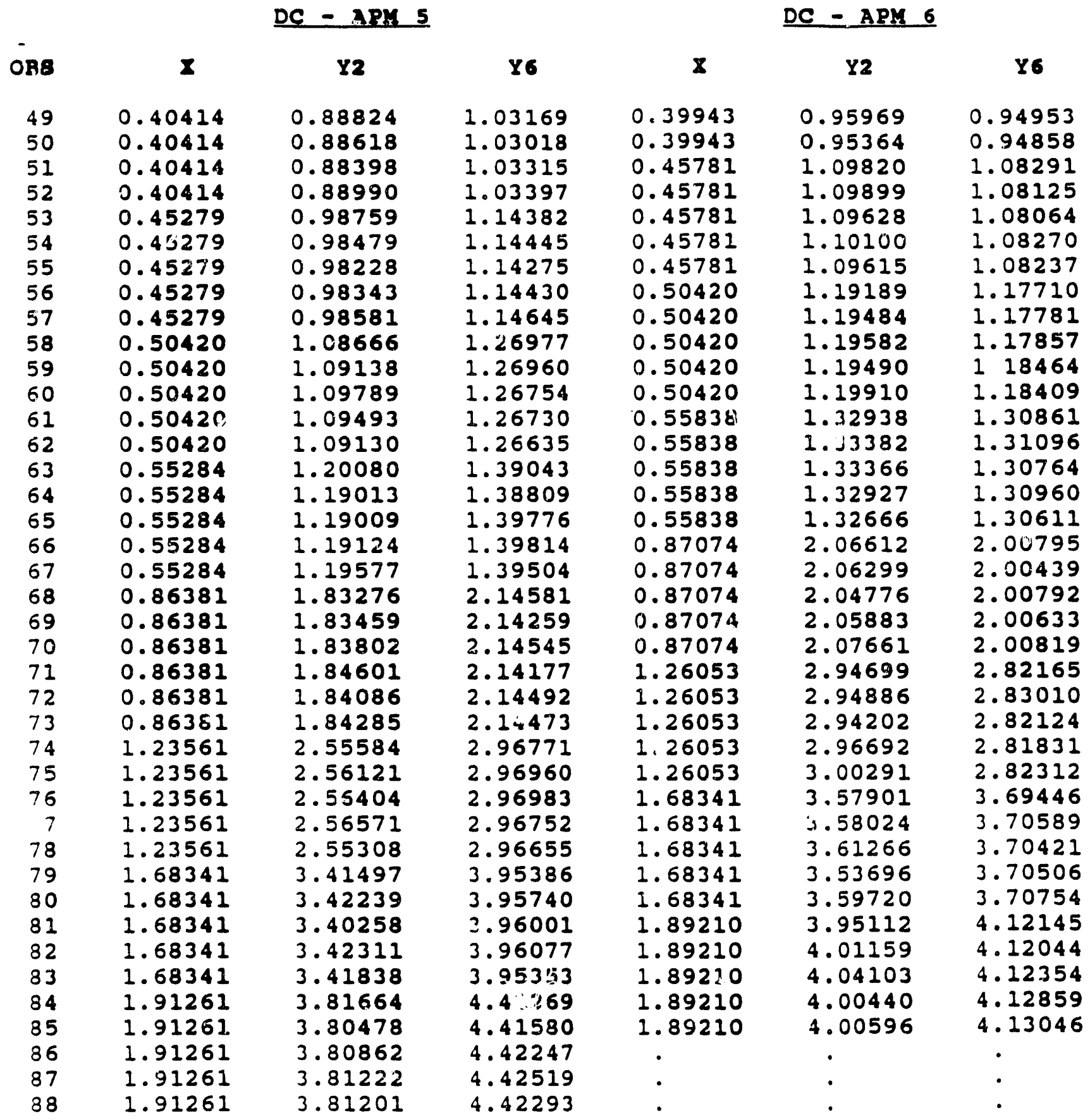


WSRC-TR-92-25

Task 92-053-1

May 29, 1992

Revision 0

Statistical Analysis of Test Data for APM Rod Issue Page 67 of 87

APPENDIX 4

DC - APY 7

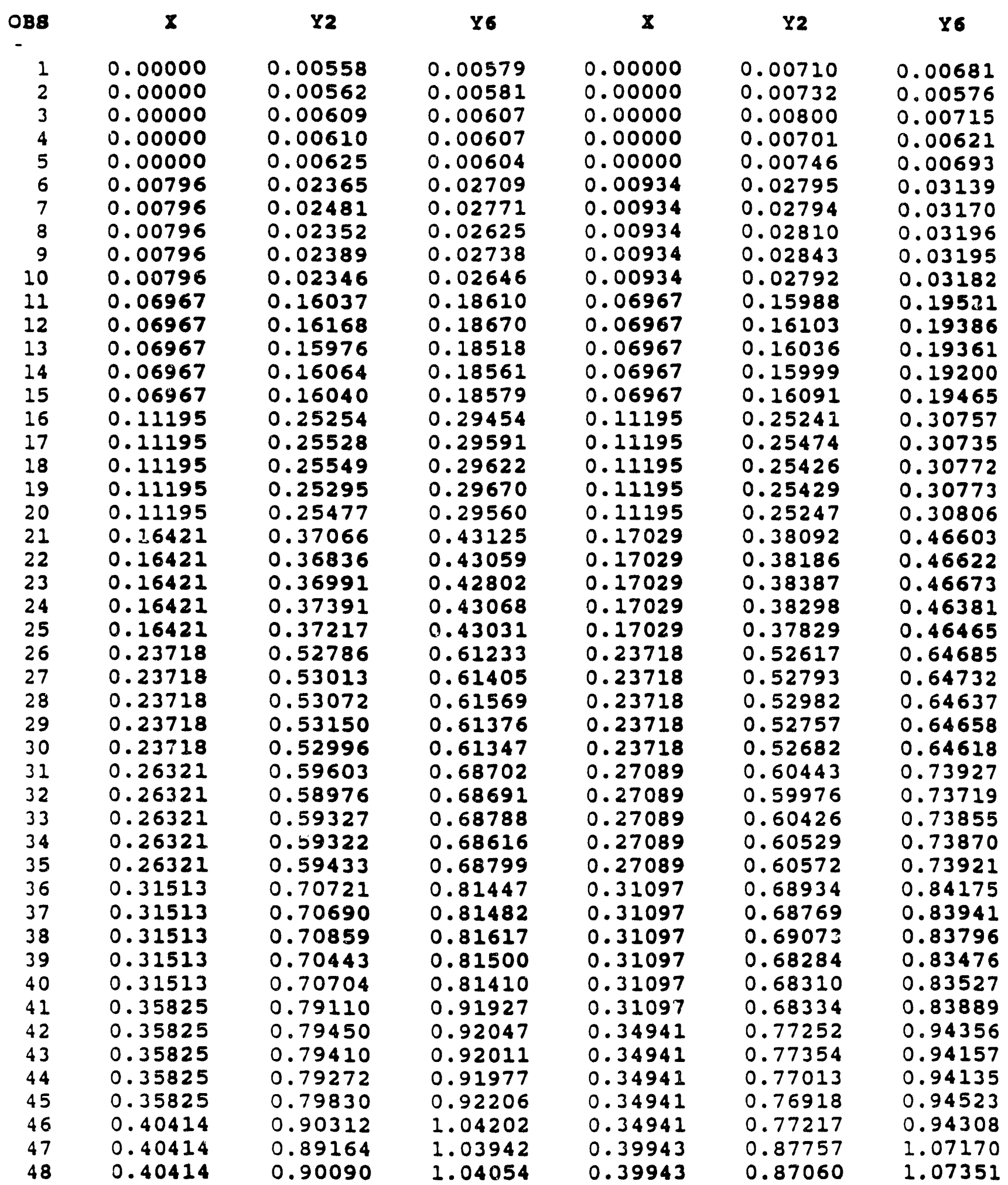


WSAC-TR-92-259

Task 92-053-1

May 29, 1992

Revision 0

Statistical Analysis of Test Data for APM Rod Issue Page 68 of 87

APPENDIX 4

( CONTINOED )

$D C-A P Y B$

Y2

Y6

0.39943

0.39943

0.45279

0.45279

0.45279

0.46792

0.45279

0.50420

0.50420

0.50420

0.50420

0.50420

0.55284

0.55284

0.55284

0.55284

0.55284

0.87074

0.87074

0.87074

0.87074

0.87074

1. 24389

1.24389

1.24389

1.24389

1. 24389

1. 68341

1.68341

1.68341

1. 68341

1.68341

1.89210

1.89210

1.89210

1.89210

1.89210

\subsection{6}

0.87172

0.87909

1. 00007

0.99667

0.98347

0.99367

0.99325

1.09472

1.09655

1.09528

1.08911

1.08735

1.20544

1.19760

1.19793

1.20906

1.20149

1.85343

1.84837

1.85687

1.84790

1.85034

2. 62202

2. 61140

2. 60849

2. 60659

2. 60640

3.44707

3.43265

3.41206

3.41817

3.44198

3.82323

3.83202

3.81414

3.81767

3.80246
1.07253

1.07197

1.07629

1.21290

1.21355

1.21005

1.21202

1. 21331 .

1. 33500

1. 33489

1.33283

1.33209

1.33423

1.46925

1.46792

1. 46811

1.46573

1.46463

2.24310

2.24585

2.25111

2.24696

2.24919

3.14586

3.14607

3.14736

3.14606

3.14897

4.11594

4.09511

4.10355

4.09903

4.10858

4.54115

4.54815

4.53484

4.54003

4.53352 
WSRC-TR-92-259

Task 92-053-1

Revision 0
Statistical Analysis of Test Data for APM Rod Issue Page 69 of 87

APPENDIX 5

$\underline{D C}-A P Y 9$

OB8

$x$

12

46

10.00000

0.00586

0.00000

0.00622

0.00000

0.00645

0.00000

0.00627

0.00000

0.00607

0.02417

0.00796

0.02449

0.00796

0.02389

0.02417

0.02409

0.16372

0.16259

0.16250

0.16327

0.01130

0.01120

0.01161

0.01145

0.01099

0.03234

0.03163

0.03193

0.03204

0.03203

0.18963

0.18942

0.18954

0.06772

0.06772

0.26394

0.11195

0.26166

0.11195

0.26151

0.11195

0.26247

0.26356

0.18995

0.30353

0.30298

0.30142

0.30434

0.30395

0.17029

0.39900

0.17029

0.17029

0.39997

0.40008

0.17029

0.39982

0.45601

0.45816

0.45868

0.45712

0.39907

0.23718

0.55313

0.23718

0.23718

0.55374

0.45708

0.63002

0.55230

0.63023

0.55093

0.62685

0.23718

0.55514

0.62745

0.23718

0.27477

0.27477

0.27477

0.63501

0.62832

0.63596

0.72010

0.63491

0.71998

0.27477

0.63413

0.72200

0.71705

0.27477

0.62904

0.71627

0.69012

0.30273

0.69345

0.78657

0.69345

0.78848

0.30273

0.69602

0.79004

0.30273

0.69258

0.78762

0.30273

0.80761

0.34941

0.80308

0.78690

0.91302

0.91264

0.34941

0.80069

0.91183

0.34941

0.80114

0.91213

0.34941

0.80892

0.91121

0.92906

0.40414

0.92949

1.05072

0.93259

1.05353

1.05304

47

0.40414

0.92760

1.05261

May 29, 1992 


\author{
APPENDIX 5 \\ ( CONTINOED)
}

$\begin{array}{cccc}\text { OB8 } & \text { X } & \text { Y2 } & Y 6 \\ 49 & 0.40414 & 0.92561 & 1.05101 \\ 50 & 0.40414 & 0.92730 & 1.05430 \\ 51 & 0.44780 & 1.01755 & 1.16030 \\ 52 & 0.44780 & 1.02409 & 1.16277 \\ 53 & 0.44780 & 1.02829 & 1.16286 \\ 54 & 0.44780 & 1.03046 & 1.15988 \\ 55 & 0.44780 & 1.02264 & 1.15776 \\ 56 & 0.50420 & 1.14966 & 1.29813 \\ 57 & 0.50420 & 1.14966 & 1.30562 \\ 58 & 0.50420 & 1.15575 & 1.29805 \\ 59 & 0.50420 & 1.14361 & 1.29757 \\ 60 & 0.50420 & 1.14283 & 1.29961 \\ 61 & 0.55838 & 1.27094 & 1.42880 \\ 62 & 0.55838 & 1.26784 & 1.42413 \\ 63 & 0.55838 & 1.26564 & 1.42882 \\ 64 & 0.55838 & 1.27965 & 1.43733 \\ 65 & 0.55838 & 1.26527 & 1.42885 \\ 66 & 0.86381 & 1.92814 & 2.17860 \\ 67 & 0.86381 & 1.93205 & 2.17430 \\ 68 & 0.86381 & 1.93533 & 2.17530 \\ 69 & 0.86381 & 1.95389 & 2.18773 \\ 70 & 0.86381 & 1.94665 & 2.18708 \\ 71 & 1.25220 & 2.75355 & 3.07376 \\ 72 & 1.25220 & 2.74452 & 3.06501 \\ 73 & 1.25220 & 2.73224 & 3.07074 \\ 74 & 1.25220 & 2.75372 & 3.08532 \\ 75 & 1.25220 & 2.77423 & 3.07988 \\ 76 & 1.69307 & 3.60446 & 4.01812 \\ 77 & 1.69307 & 3.62651 & 4.03858 \\ 78 & 1.69307 & 3.61010 & 4.01441 \\ 79 & 1.69307 & 3.61389 & 4.02586 \\ 80 & 1.69307 & 3.62974 & 4.04058 \\ 81 & 1.89210 & 4.01217 & 4.44370 \\ 82 & 1.89210 & 3.96435 & 4.43734 \\ 83 & 1.89210 & 3.97758 & 4.42909 \\ 84 & 1.89210 & 3.99025 & 4.45426 \\ 85 & 1.89210 & 3.99245 & 4.44472\end{array}$


WSRC-TR-92-259

Task 92-053-1

May 29, 1992

Revision 0

Statistical Analysis of Test Data for APM Rod Issue Page 71 of 87

\section{APPENDIX 6}

AC - APX 1

OB8

2

3

4

5

6

8

9

10

11

12

13

14

15

16

17

18

19

20

21

22

23

24

25

26

27

28

29

30

31

32

33

34

35
Y2

0.00000
0.00000
0.00000
0.00000
0.00000
0.00864
0.00864
0.00864

0.00864

0.00864

0.06772

0.06772

0.06772

0.06772

0.06772

0.11195

0.11195

0.11195

0.11195

0.11195

0.13821

0.13821

0.13821

0.13821

0.13821

0.23358

0.23358

0.23358

0.23358

0.23358

0.27089

0.27089

0.27089

0.27089

0.27089

0.27089

0.31097

0.31097

0.31097

0.31097

0.31097

0.35382

0.35382

0.35382

0.35382

0.35382

0.39474

0.39474
0.01860

0.00493

0.00539

0.00475

0.00519

0.02514

0.02525

0.02535

0.02516

0.02502

0.15338

0.15440

0.15632

0.15557

0.15488

0.25852

0.25776

0.25813

0.27118

0.25956

0.31275

0.31346

0.31403

0.32690

0.53136

0.53342

0.52962

0.54325

0.53464

0.61408

0.60936

0.61607

0.61278

0.61430

0.61600

0.69652

0.71504

0.70007

0.69777

0.69668

0.79095

0.79476

0.79478

0.80454

0.78938

0.88093

0.88650
0.31339
$\mathbf{Y 6}$

0.02696

0.00426

0.00432

0.00393

0.00477

0.02610

0.02612

0.02605

0.02625

0.02594

0.16452

0.16574

0.16712

0.16744

0.16760

0.27813

0.27719

0.27727

0.29998

0.27869

0.33704

0.33754

0.33728

0.36020

0.33618

0.57489

0.57427

0.57248

0.59580

0.57499

0.66131

0.65969

0.66433

0.66334

0.66279

0.66235

0.75321

0.77695

0.75281

0.75161

0.75058

0.85418

0.85694

0.85580

0.87516

0.85203

0.94818

0.96550
AC - APX 2

\section{8}

12

16

0.00000
0.00000
0.00000
0.00000
0.00000
0.00864
0.00864

0.00864

0.00864

0.00864

0.06772

0.06772

0.06772

0.06772

0.06772

0.11195

0.11195

0.11195

0.11195

0.11195

0.14099

0.14099

0.14099

0.14099

0.14099

0.23718

0.23718

0.23718

0.23718

0.23718

0.27089

0.27089

0.27089

0.27089

0.27089

0.31097

0.31097

0.31097

0.31097

0.31097

0.35382

0.35382

0.35382

0.35382

0.35382

0.39474

0.39474

0.39474

0.00342

0.00373

0.00361

0.00370

0.00378

0.02532

0.02588

0.02515

0.02566

0.02607

0.16190

0.16195

0.16223

0.16202

0.16218

0.27024

0.26988

0.27075

0.26947

0.26949

0.33567

0.33504

0.33415

0.33610

0.33525

0.59352

0.59657

0.59623

0.59158

0.59594

0.63762

0.64034

0.63934

0.64379

0.64050

0.72484

0.72559

0.72452

0.72472

0.72659

0.83174

0.83262

0.83346

0.82879

0.82932

0.91926 
WSRC-TR-92-259

Task 92-053-1

May 29, 1992

Revision 0

Statlstlcal Analysis of Test Data for APM Rod Issue Page 72 of 87

\section{APPENDIX 6}

( CONTINOED)

\begin{tabular}{|c|c|c|c|c|c|c|}
\hline \multirow{2}{*}{ OBs } & \multicolumn{3}{|c|}{$A C-A P Y 1$} & \multicolumn{3}{|c|}{$A C-A P X 2$} \\
\hline & $\mathbf{z}$ & $\mathbf{Y 2}$ & $Y 6$ & $\mathbf{x}$ & $Y 2$ & $Y 6$ \\
\hline $\begin{array}{l}49 \\
50 \\
51 \\
52 \\
53 \\
54 \\
55 \\
56 \\
57 \\
58 \\
59 \\
60 \\
61 \\
62 \\
63 \\
64 \\
65 \\
66 \\
67 \\
68 \\
69 \\
70 \\
71 \\
72 \\
73 \\
74 \\
75 \\
76 \\
77 \\
78 \\
79 \\
80 \\
81 \\
82 \\
83 \\
84 \\
85\end{array}$ & $\begin{array}{l}0.39474 \\
0.39474 \\
0.39474 \\
0.44780 \\
0.44780 \\
0.44780 \\
0.44780 \\
0.44780 \\
0.55284 \\
0.55284 \\
0.55284 \\
0.55284 \\
0.55284 \\
0.86381 \\
0.86381 \\
0.86381 \\
0.86381 \\
0.86381 \\
1.23561 \\
1.23561 \\
1.23561 \\
1.23561 \\
1.23561 \\
1.69307 \\
1.69307 \\
1.69307 \\
1.69307 \\
1.69307 \\
1.89210 \\
1.89210 \\
1.89210 \\
1.89210 \\
1.89210 \\
. \\
.\end{array}$ & $\begin{array}{l}0.87495 \\
0.87769 \\
0.88581 \\
1.00241 \\
1.00021 \\
1.00950 \\
0.99640 \\
1.00063 \\
1.21454 \\
1.21200 \\
1.21579 \\
1.21592 \\
1.21666 \\
1.88111 \\
1.89013 \\
1.88411 \\
1.88951 \\
1.89360 \\
2.65723 \\
2.64293 \\
2.64675 \\
2.64809 \\
2.65953 \\
3.56336 \\
3.55909 \\
3.57587 \\
3.58543 \\
3.58562 \\
3.95488 \\
3.95593 \\
3.95138 \\
3.95090 \\
3.94222 \\
. \\
. \\
. \\
.\end{array}$ & $\begin{array}{l}0.94689 \\
0.95076 \\
0.95437 \\
1.08176 \\
1.08141 \\
1.08656 \\
1.07903 \\
1.08139 \\
1.31295 \\
1.31335 \\
1.31573 \\
1.31404 \\
1.31519 \\
2.03873 \\
2.04036 \\
2.03917 \\
2.04214 \\
2.04494 \\
2.86931 \\
2.84799 \\
2.85115 \\
2.85335 \\
2.87854 \\
3.84814 \\
3.84073 \\
3.85009 \\
3.84832 \\
3.85010 \\
4.26038 \\
4.25856 \\
4.25962 \\
4.25289 \\
4.25687 \\
. \\
.\end{array}$ & $\begin{array}{l}0.39474 \\
0.39474 \\
0.45279 \\
0.45279 \\
0.45279 \\
0.45279 \\
0.45279 \\
0.49894 \\
0.49894 \\
0.49894 \\
0.49894 \\
0.49894 \\
0.55838 \\
0.55838 \\
0.55838 \\
0.55838 \\
0.55838 \\
0.86381 \\
0.86381 \\
0.86381 \\
0.86381 \\
0.86381 \\
1.23561 \\
1.23561 \\
1.23561 \\
1.23561 \\
1.23561 \\
1.69307 \\
1.69307 \\
1.69307 \\
1.69307 \\
1.69307 \\
1.90234 \\
1.90234 \\
1.90234 \\
1.90234 \\
1.90234\end{array}$ & $\begin{array}{l}0.91951 \\
0.91881 \\
1.05211 \\
1.05166 \\
1.05299 \\
1.05391 \\
1.05299 \\
1.13943 \\
1.13966 \\
1.13598 \\
1.13613 \\
1.14113 \\
1.29840 \\
1.29454 \\
1.29502 \\
1.29822 \\
1.29387 \\
1.97280 \\
1.97463 \\
1.98063 \\
1.98158 \\
1.98408 \\
2.79280 \\
2.79557 \\
2.79045 \\
2.80623 \\
2.79452 \\
3.71941 \\
3.72698 \\
3.72930 \\
3.72870 \\
3.72892 \\
4.14961 \\
4.16781 \\
4.17151 \\
4.14346 \\
4.14823\end{array}$ & $\begin{array}{l}0.90241 \\
0.90217 \\
1.03459 \\
1.03295 \\
1.03424 \\
1.03522 \\
1.03500 \\
1.11961 \\
1.11874 \\
1.11670 \\
1.11847 \\
1.12149 \\
1.27458 \\
1.26883 \\
1.27429 \\
1.27418 \\
1.27090 \\
1.94070 \\
1.94408 \\
1.94915 \\
1.94758 \\
1.94880 \\
2.75145 \\
2.74824 \\
2.74352 \\
2.75149 \\
2.74659 \\
3.66354 \\
3.66977 \\
3.66580 \\
3.67296 \\
3.67277 \\
4.08760 \\
4.09760 \\
4.10110 \\
4.09339 \\
4.09418\end{array}$ \\
\hline
\end{tabular}


WSRC.TR-92-259

Task 92-053-1

May 29, 1992

Revision 0

Statistical Analysis of Test Data for APM Rod Issue Page 73 of 87

\section{APPENDIX 7}

$A C-A P \times 3$

OB8

2

3 $x$

0.00000

0.00000

0.00000

0.00000

0.00000

0.00864

0.00864

0.00864

0.00864

0.00864

0.06580

0.06580

0.06580

0.06580

0.06580

0.11195

0.11195

0.11195

0.11195

0.11195

0.13821

0.13821

0.13821

0.13821

0.13821

0.23358

0.23358

0.23358

0.23358

0.23358

0.27089

0.27089

0.27089

0.27089

0.27089

0.30684

0.30684

0.30684

0.30684

0.30684

0.35825

0.35825

0.35825

0.35825

0.35825

0.40414

0.40414

0.40414
Y2

0.00473

0.00476

0.00490

0.00464

0.00432

0.02454

0.02489

0.02474

0.02455

0.02484

0.15724

0.15677

0.15693

0.15743

0.15743

0.26720

0.26681

0.26754

0.26673

0.26718

0.32358

0.32336

0.32372

0.32206

0.32240

0.54571

0.54600

0.54646

0.54760

0.54731

0.62416

0.62265

0.62428

0.62666

0.70966

0.70747

0.71092

0.70955

0.71132

0.81895

0.81757

0.81985

0.92649

0.92656

0.92226
0.62582

0.81848

0.82061
Y6

0.00418

0.00416

0.00433

0.00397

0.00405

0.02432

0.02418

0.02415

0.02434

0.02464

0.15651

0.15653

0.15528

0.15679

0.15624

0.26624

0.26583

0.26606

0.26576

0.26613

0.32228

0.32125

0.32210

0.32035

0.32074

0.54384

0.54417

0.54499

0.54476

0.54407

0.62169

0.62226

0.62189

0.62250

0.62265

0.70569

0.70585

0.70757

0.70695

0.70802

0.81580

0.81529

0.81555

0.81674

0.81729

0.92108

0.92023

0.91900

$A C$ - APM 4

$x$

Y2

Y6

\subsection{0}

0.00000

0.00000

0.00000

0.00000

0.00864

0.00864

0.00864

0.00864

0.00864

0.06580

0.06580

0.06580

0.06580

0.06580

0.11195

0.11195

0.11195

0.11195

0.11195

0.13821

0.13821

0.13821

0.13821

0.13821

0.23718

0.23718

0.23718

0.23718

0.23718

0.27089

0.27089

0.27089

0.27089

0.27089

0.31513

0.31513

0.31513

0.31513

0.31513

0.35382

0.35382

0.35382

0.35382

0.35382

0.39943

0.39943

0.39943

0.00444

0.00490

0.00459

0.00457

0.00443

0.02354

0.02422

0.02470

0.02434

0.02444

0.15091

0.15190

0.15131

0.15124

0.15186

0.25800

0.25772

0.25756

0.25815

0.25815

0.31147

0.31319

0.31256

0.31227

0.31122

0.52735

0.52732

0.52696

0.52601 
WSRC-TR-92-259

Task 92-053-1

May 29, 1992

Revision 0

Statistical Analysis of Test Data for APM Rod Issue Page 74 of 87

\author{
APPENDIX 7 \\ ( CONTINOED )
}

\begin{tabular}{|c|c|c|c|c|c|c|}
\hline \multirow{2}{*}{$\overline{\text { OBB }}$} & \multicolumn{3}{|c|}{$A C-A P Y 3$} & \multicolumn{3}{|c|}{$A C-A P M 4$} \\
\hline & $\mathbf{z}$ & $\mathbf{Y 2}$ & Y6 & $x$ & $Y 2$ & Y6 \\
\hline $\begin{array}{l}49 \\
50 \\
51 \\
52 \\
53 \\
54 \\
55 \\
56 \\
57 \\
58 \\
59 \\
60 \\
61 \\
62 \\
63 \\
64 \\
65 \\
66 \\
67 \\
68 \\
69 \\
70 \\
71 \\
72 \\
73 \\
74 \\
75 \\
76 \\
77 \\
78 \\
79 \\
80 \\
81 \\
82 \\
83 \\
84 \\
85 \\
86\end{array}$ & $\begin{array}{l}0.40414 \\
0.40414 \\
0.44780 \\
0.44780 \\
0.44780 \\
0.44780 \\
0.44780 \\
0.49370 \\
0.49370 \\
0.49370 \\
0.49370 \\
0.49370 \\
0.55838 \\
0.55838 \\
0.55838 \\
0.55838 \\
0.55838 \\
0.87074 \\
0.87074 \\
0.87074 \\
0.87074 \\
0.87074 \\
1.25220 \\
1.25220 \\
1.25220 \\
1.25220 \\
1.25220 \\
1.70276 \\
1.70276 \\
1.70276 \\
1.70276 \\
1.70276 \\
1.89210 \\
1.89210 \\
1.89210 \\
1.89210 \\
1.89210\end{array}$ & $\begin{array}{l}0.92249 \\
0.92441 \\
1.02527 \\
1.02257 \\
1.02338 \\
1.02549 \\
1.02520 \\
1.12650 \\
1.12891 \\
1.13718 \\
1.13060 \\
1.12685 \\
1.27777 \\
1.27659 \\
1.27571 \\
1.27955 \\
1.27966 \\
1.95004 \\
1.94822 \\
1.95724 \\
1.95441 \\
1.94737 \\
2.77351 \\
2.77525 \\
2.77563 \\
2.77720 \\
2.77263 \\
3.70019 \\
3.69803 \\
3.69136 \\
3.68832 \\
3.70415 \\
4.05605 \\
4.06404 \\
4.07139 \\
4.05490 \\
4.05750\end{array}$ & $\begin{array}{l}0.91955 \\
0.92128 \\
1.01973 \\
1.01779 \\
1.01796 \\
1.02053 \\
1.02065 \\
1.12441 \\
1.12778 \\
1.12975 \\
1.12433 \\
1.12404 \\
1.27203 \\
1.27149 \\
1.27187 \\
1.27387 \\
1.27459 \\
1.94573 \\
1.94662 \\
1.95251 \\
1.94708 \\
1.94370 \\
2.76995 \\
2.76982 \\
2.76823 \\
2.76834 \\
2.76960 \\
3.69095 \\
3.68565 \\
3.68505 \\
3.68551 \\
3.68986 \\
4.05437 \\
4.05600 \\
4.05390 \\
4.04918 \\
4.05666 \\
.0\end{array}$ & $\begin{array}{l}0.39943 \\
0.39943 \\
0.44284 \\
0.44284 \\
0.44284 \\
0.44284 \\
0.44284 \\
0.50420 \\
0.50420 \\
0.50420 \\
0.50420 \\
0.50420 \\
0.55284 \\
0.55284 \\
0.55284 \\
0.55284 \\
0.55284 \\
0.55284 \\
0.86381 \\
0.86381 \\
0.86381 \\
0.86381 \\
0.86381 \\
1.24389 \\
1.24389 \\
1.24389 \\
1.24389 \\
1.24389 \\
1.69307 \\
1.69307 \\
1.69307 \\
1.69307 \\
1.69307 \\
1.90234 \\
1.90234 \\
1.90234 \\
1.90234 \\
1.90234\end{array}$ & $\begin{array}{l}0.88708 \\
0.88326 \\
0.97706 \\
0.97976 \\
0.98038 \\
0.97944 \\
0.98026 \\
1.11667 \\
1.11490 \\
1.11512 \\
1.11508 \\
1.11614 \\
1.21700 \\
1.22520 \\
1.21893 \\
1.22492 \\
1.22636 \\
1.22625 \\
1.87865 \\
1.88181 \\
1.88083 \\
1.88303 \\
1.88168 \\
2.66785 \\
2.66912 \\
2.67874 \\
2.66450 \\
2.66961 \\
3.54171 \\
3.54131 \\
3.55740 \\
3.55007 \\
3.54940 \\
3.94642 \\
3.95235 \\
3.94138 \\
3.94598 \\
3.94143\end{array}$ & $\begin{array}{l}0.96749 \\
0.96648 \\
1.06800 \\
1.07008 \\
1.07074 \\
1.07145 \\
1.07213 \\
1.21931 \\
1.21699 \\
1.21685 \\
1.21705 \\
1.21865 \\
1.33308 \\
1.33627 \\
1.33682 \\
1.33788 \\
1.33983 \\
1.33801 \\
2.05844 \\
2.05822 \\
2.05699 \\
2.05638 \\
2.05105 \\
2.91396 \\
2.91439 \\
2.91668 \\
2.91573 \\
2.91738 \\
3.86986 \\
3.87212 \\
3.87749 \\
3.87737 \\
3.86912 \\
4.31083 \\
4.31034 \\
4.30461 \\
4.30190 \\
4.22531\end{array}$ \\
\hline
\end{tabular}


WSRC-TR-92-259

Task 92-053-1

May 29, 1992

Statistical Analysis of Test Data for APM Rod Issue

Revision 0

Page 75 of 87

APPENDIX 8

$A C-A P X 5$

OBs

$$
1
$$

\section{I}

0.00000

0.00000

0.00000

0.00000

0.00000

0.00000

0.00934

0.00934

0.00934

0.00934

0.00934

0.06967

0.06967

0.06967

0.06967

0.06967

0.11445

0.11445

0.11445

0.11445

0.11445

0.13821

0.13821

0.13821

0.13821

0.23000

0.23000

0.23000

0.23000

0.23000

0.27089

0.27089

0.27089

0.27089

0.27089

0.31513

0.31513

0.31513

0.31513

0.31513

0.35382

0.35382

0.35382

0.35382

0.35382

0.39943

ग. 39943
0.13821
$Y 2$

0.00637

0.00598

0.00596

0.00581

0.00593

0.00592

0.02615

0.02691

0.02664

0.02655

0.02629

0.15820

0.15849

0.15896

0.15864

0.15896

0.25188

0.25176

0.25206

0.25209

0.25176

0.30549

0.30480

0.30619

0.30522

0.30516

0.50021

0.50183

0.50033

0.49952

0.50061

0.59331

0.59238

0.59201

0.59372

0.59444

0.68495

0.68354

0.68226

0.68371

0.68468

0.76838

0.76788

0.76906

0.77065

0.77041

0.86306

0.86317
Y6

0.00596

0.00519

0.00508

0.00526

0.00532

0.00553

0.02659

0.02794

0.02719

0.02742

0.02752

0.17018

0.17017

0.17037

0.16995

0.17061

0.27007

0.27111

0.27063

0.27058

0.27102

0.32825

0.32820

0.32954

0.32870

0.32881

0.53933

0.53976

0.53853

0.53953

0.53994

0.63840

0.63809

0.63991

0.63934

0.63913

0.73706

0.73681

0.73730

0.73848

0.73843

0.82919

0.82842

0.82972

0.83021

0.83032

0.92980

0.92925
$A C$ - $A D M 6$

x

Y2

Y6

0.00000

0.00000

0.00000

0.00000

0.00000

0.00864

0.00864

0.00864

0.00864

0.06967

0.06967

0.06967

0.06967

0.11445

0.11445

0.11445

0.11445

0.11445

0.13821

0.13821

0.13821

0.13821

0.13821

0.23358

0.23358

0.23358

0.23358

0.23358

0.27089

0.27089

0.27089

0.27089

0.27089

0.30684

0.30684

0.30684

0.30684

0.30684

0.30684

0.35382

0.35382

0.35382

0.35382

0.35382

0.39943

0.39943

0.39943

0.39943

0.00554

0.00642

0.00565

0.00590

0.02582

0.02555

0.02597

0.02588

0.16378

0.16359

0.16379

0.16312

0.26524

0.26539

0.26638

0.26516

0.26497

0.32007

0.32017

0.32038

0.32004

0.32052

0.54082

0.54149

0.54080

0.54128

0.54317

0.61840 
WSRC-TR-92-259

Task 92-053-1

May 29, 1992

Revision 0

Statistical Analysis of Test Data for APM Rod Issue Page 76 of 87

\section{APPENDIX 8}

CONTINOED )
AC - APY 5

OB8

49

50

51

52

53

54

55

56

57

58

59

60

61

62

63

64

65

66

67

68

69

70

71

72

73

74

75

76

77

78

79

80

81

82

83

84

85

86
0.39943

0.39943

0.39943

0.44284

0.44284

0.44284

0.44284

0.44284

0.49370

0.49370

0.49370

0.49370

0.49370

0.54732

0.54732

0.54732

0.54732

0.86381

0.86381

0.86381

0.86381

0.86381

1. 23561

1. 23561

1. 23561

1.23561

1. 23561

1.70276

1.70276

1.70276

1.70276

1.70276

1.89210

1.89210

1.89210

1.89210

1.89210
0.54732
0.86168

0.86191

0.86140

0.95790

0.95896

0.95837

0.95965

0.95915

1.06340

1.06143

1.06365

1.06077

1. 06261

1.17120

1. 17232

1.16774

1. 17001

1.16686

1.82518

1.82542

1.82766

1. 83209

1.83109

2. 57371

2. 57919

2. 57848

2. 58437

2. 57917

3.49159

3.49042

3.48663

3.48043

3. 48380

3.83565

3.83083

3.83587

3. 83373

3.83299

0.93033
0.93022
0.93091
1.03397
1.03402
1.03418
1.03433
1.03437
1.14485
1.14574
1.14549
1.14500
1.14441
1.26127
1.26056
1.25869
1.25912
1.25765
1.96973
1.97043
1.97167
1.97192
1.97124
2.77288
2.77858
2.78353
2.78047
2.77587
3.75786
3.75523
3.75258
3.75263
3.75735
4.13017
4.13790
4.13717
4.13198
4.13061

0.39943

0.44780

0.44780

0.44780

0.44780

0.44780

0.49370

0.49370

0.49370

0.49370

0.49370

0.55284

0.55284

0.55284

0.55284

0.55284

0.87074

0.87074

0.87074

0.87074

0.87074

1.24389

1.24389

1.24389

1.24389

1.24389

1.70276

1.70276

1.70276

1.70276

1.70276

1.89210

1.89210

1.89210

1.89210

1.89210

AC - APY 6

$Y 2$

$\mathbf{Y 6}$

0.92195

1.00820

1.02077

1.00689

1.00770

1.00968

1. 00938

1. 11326

1. 11453

1. 11307

1. 11141

1. 11137

1.24420

1. 24341

1.24409

1.24576

1. 24480

1.94246

1. 94713

1.94600

1.94585

1.94835

2. 73024

2. 73419

2. 73496

2.73103

2. 73452

3.65275

3.65021

3. 65181

3.65056

3. 65361

4. 00517

4.00802

4.00943

4.02234

4.01344
1.02053

1.02002

1.02114

1.02200

1. 12881

1. 12789

1. 12705

1. 12627

1.12539

1. 25947

1. 25880

1. 26014

1.26204

1. 26063

1.96904

1. 97113

1.97077

1.96982

1.97317

2. 76984

2.77028

2.77399

2.77044

2.77128

3.71036

3.70800

3.70562

3.70424

3.70775

4.07103

4.07136

4.07489

4.07915

4.07488 
WSRC-TR-92-259

Task 92-053-1

Statistical Analysis of Test Data for APM Rod Issue

May 29, 1992

Revision 0

Page 77 of 87

\author{
APPENDIX 9
}

$A C-A P Y 7$

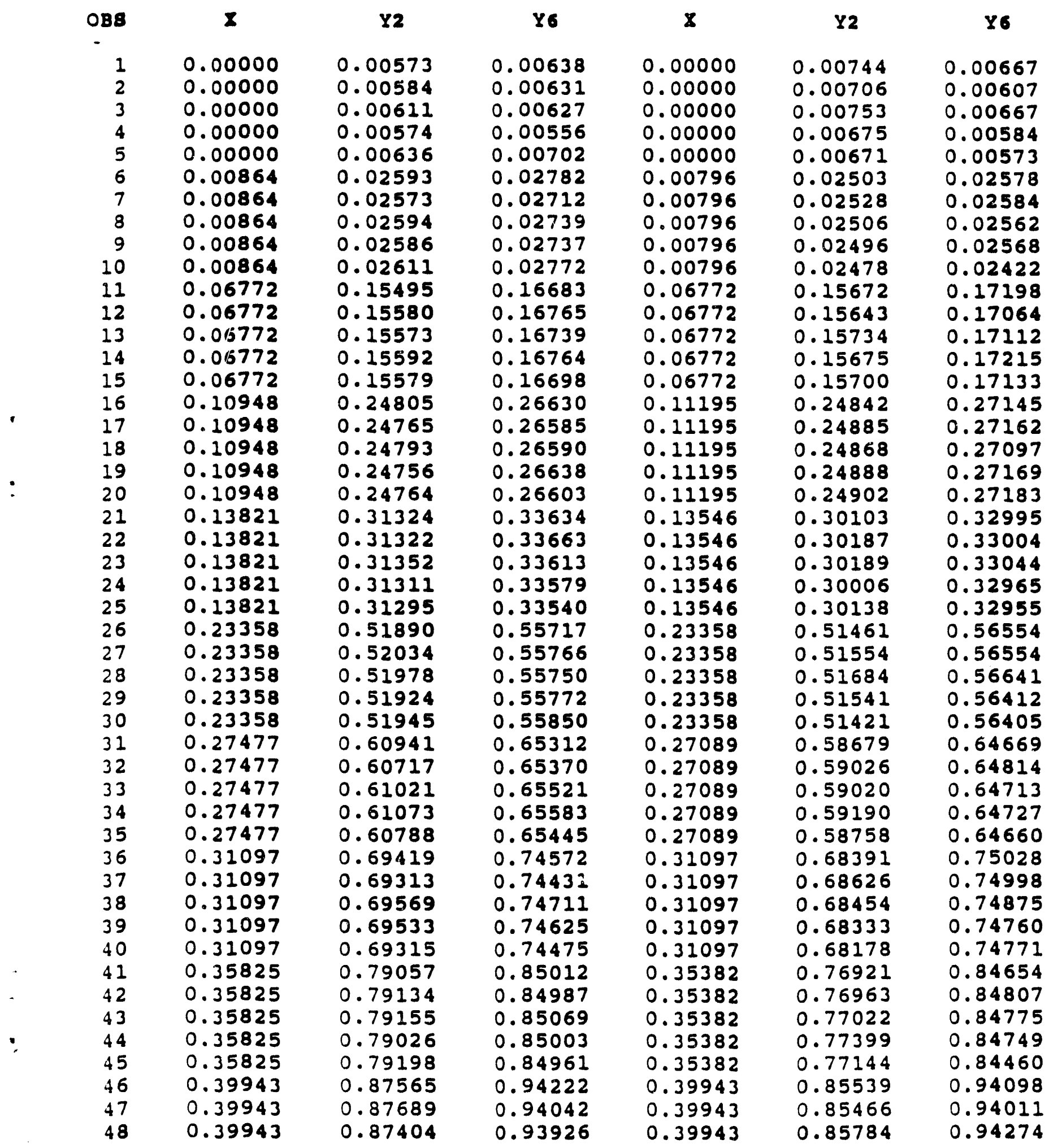

$A C$ - APY 8 
WSRC-TR-92-259

Task 92-053-1

Statistlcal Analysis of Test Data for APM Rod Issue Page 78 of 87

May 29, 1992

Revision 0

\section{APPGNDTX 9}

( CONTINOED )

$A C$ - APY 7

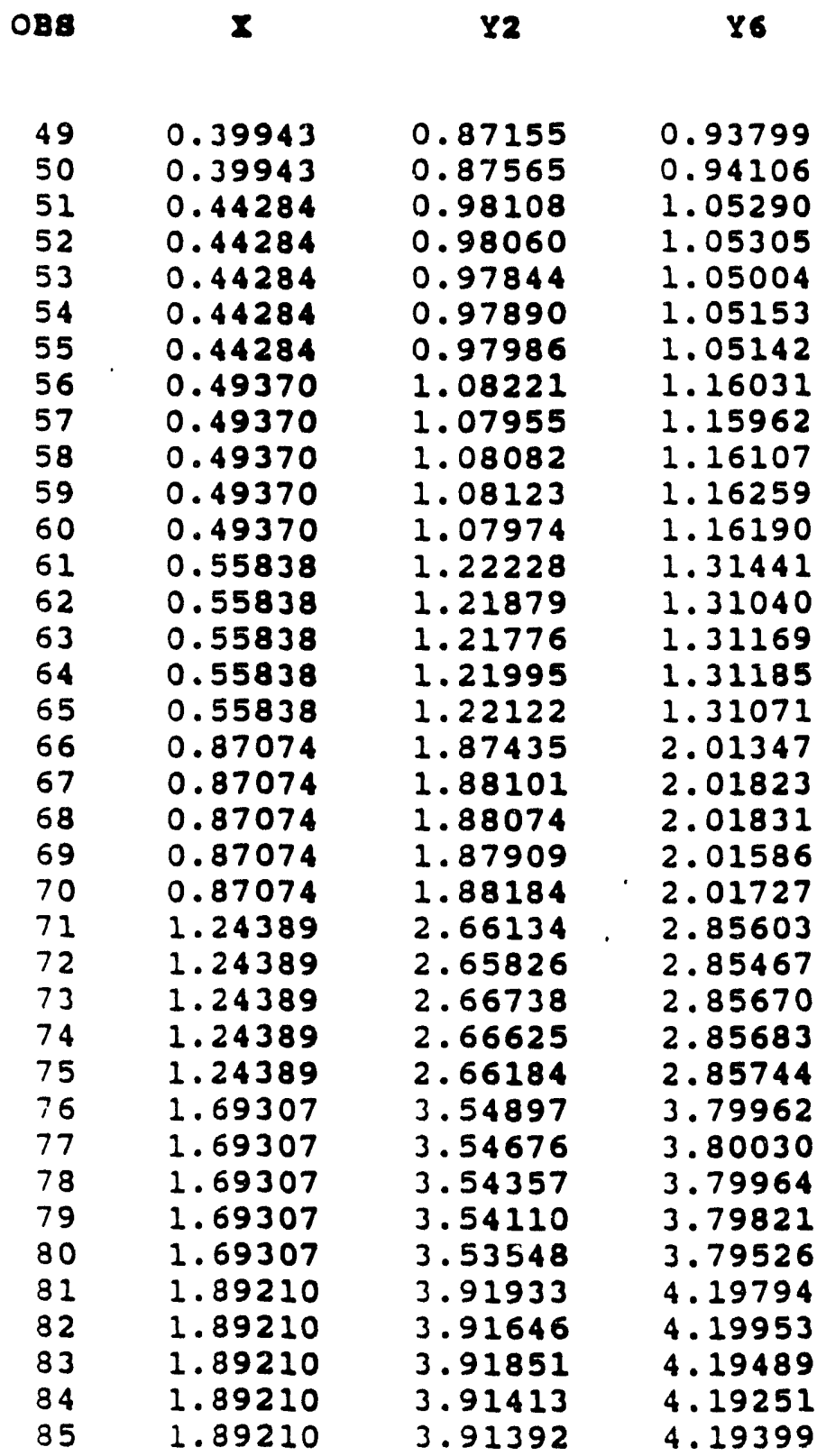

$A C$ - ADY 8

x

Y2

Y6

0.39943

0.85929

0.85983

0.96413

0.96434

0.96458

0.96398

0.96337

1.06150

1.06256

1.06235

1. 05983

1.06242

1.19775

1. 19838

1. 18905

1. 19080

1. 19615

1.84916

1.85486

1.85347

1.85224

1. 84170

2. 60995

2.61422

2. 61454

2. 60001

2. 59973

3.48889

3.48023

3. 48751

3. 49187

3.47859

3.84431

3.83877

3.82203

3.83094

3.82679
0.94119

0.93968

1.05861

1.05620

1.05662

1. 05631

1.05599

1. 16666

1.16535

1. 16615

1. 16572

1. 16579

1.31342

1. 31266

1. 30761

1. 30962

1. 31295

2. 02927

2. 03207

2. 03146

2.03264

2.03012

2. 86268

2.86156

2.86126

2.85885

2.86174

3.81610

3.81633

3.81276

3.82463

3. 81800

4.21306

4. 20206

4. 19730

4.19984

4.20469 
APPFNDIX 10

AC - APY?

$\begin{array}{cccc}\text { OB8 } & \text { X } & \text { Y2 } & Y \\ & & & \\ 1 & 0.00000 & 0.00562 & 0.01165 \\ 2 & 0.00000 & 0.00549 & 0.01127 \\ 3 & 0.00000 & 0.00568 & 0.01137 \\ 4 & 0.00000 & 0.00584 & 0.01137 \\ 5 & 0.00000 & 0.00594 & 0.01148 \\ 6 & 0.00864 & 0.02519 & 0.03158 \\ 7 & 0.00864 & 0.02565 & 0.03150 \\ 8 & 0.00864 & 0.02622 & 0.03251 \\ 9 & 0.00864 & 0.02592 & 0.03270 \\ 10 & 0.00864 & 0.02628 & 0.03236 \\ 11 & 0.06772 & 0.16378 & 0.17515 \\ 12 & 0.06772 & 0.16416 & 0.17592 \\ 13 & 0.06772 & 0.16551 & 0.17603 \\ 14 & 0.06772 & 0.16568 & 0.17769 \\ 15 & 0.06772 & 0.16520 & 0.17716 \\ 16 & 0.11445 & 0.26813 & 0.28293 \\ 17 & 0.11195 & 0.26769 & 0.28343 \\ 18 & 0.11195 & 0.26921 & 0.28392 \\ 19 & 0.11195 & 0.26866 & 0.28312 \\ 20 & 0.11195 & 0.26951 & 0.28390 \\ 21 & 0.13821 & 0.32391 & 0.34086 \\ 22 & 0.13821 & 0.32505 & 0.34032 \\ 23 & 0.13821 & 0.32420 & 0.34027 \\ 24 & 0.13821 & 0.32377 & 0.34044 \\ 25 & 0.13821 & 0.32520 & 0.34049 \\ 26 & 0.23358 & 0.54991 & 0.57325 \\ 27 & 0.23358 & 0.54800 & 0.57265 \\ 28 & 0.23358 & 0.54837 & 0.57225 \\ 29 & 0.23358 & 0.54816 & 0.57187 \\ 30 & 0.23358 & 0.54878 & 0.57384 \\ 31 & 0.27089 & 0.62827 & 0.65504 \\ 32 & 0.27089 & 0.62709 & 0.65500 \\ 33 & 0.27089 & 0.62931 & 0.65686 \\ 34 & 0.27089 & 0.62989 & 0.65636 \\ 35 & 0.27089 & 0.62878 & 0.65595 \\ 36 & 0.30684 & 0.71756 & 0.74630 \\ 37 & 0.30684 & 0.71312 & 0.74291 \\ 38 & 0.30684 & 0.71230 & 0.74071 \\ 39 & 0.30684 & 0.71261 & 0.74128 \\ 40 & 0.30684 & 0.71346 & 0.74089 \\ 41 & 0.30684 & 0.71420 & 0.74200 \\ 42 & 0.35382 & 0.82060 & 0.88419 \\ 43 & 0.35382 & 0.82317 & 0.85654 \\ 44 & 0.35382 & 0.82475 & 0.85746 \\ 45 & 0.35382 & 0.82561 & 0.85748 \\ 46 & 0.35382 & 0.82518 & 0.85753 \\ 47 & 0.39943 & 0.92668 & 0.96289\end{array}$


APPENDIX 10

( CONTINOED )

AC - APY 9

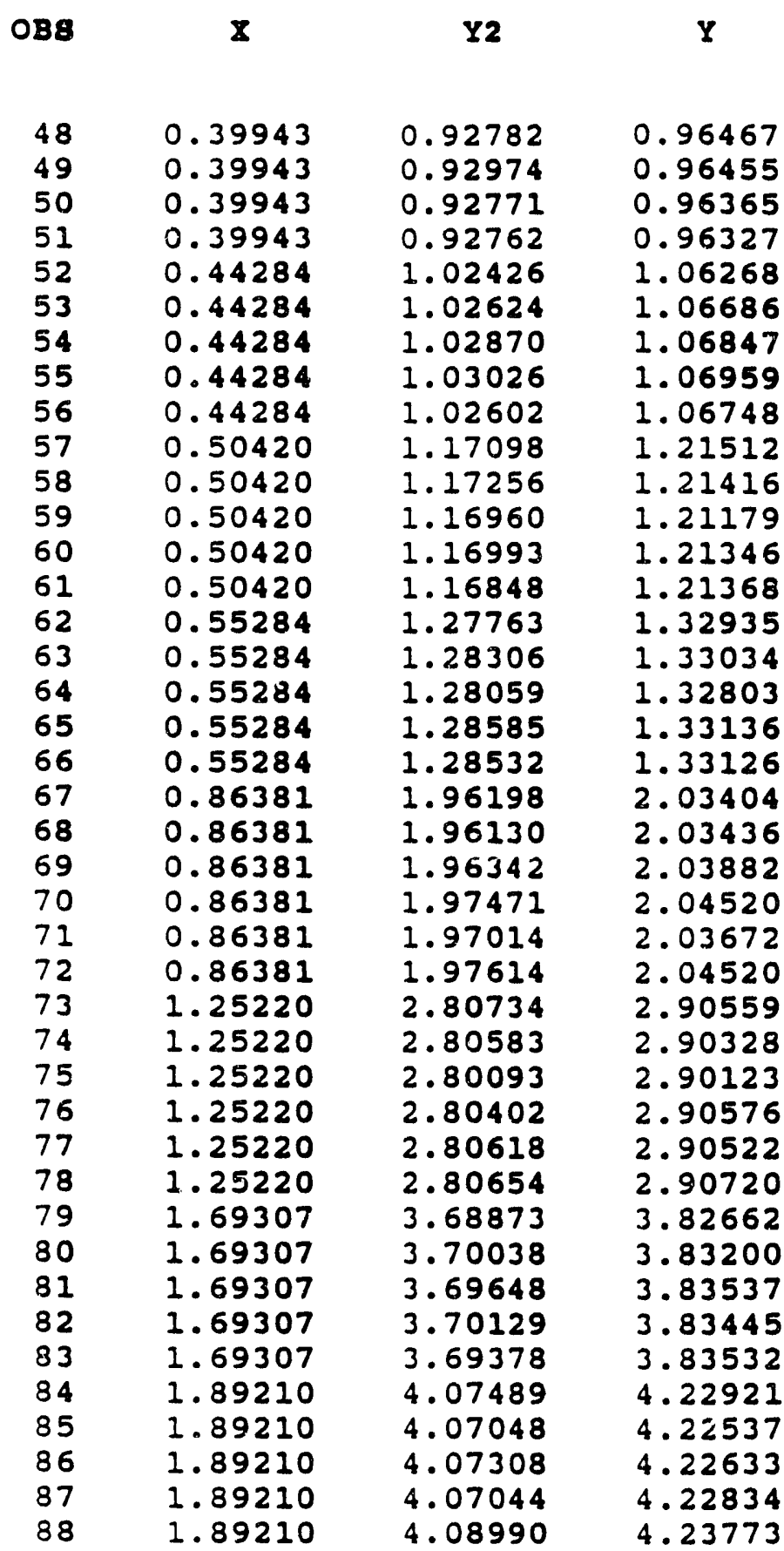


WSRC-TR-92-259

Task 92-053-1

May 29, 1992

Revision 0

Statistlcal Analysis of Test Data for APM Rod Issue Pagr 81 of 87

\author{
APPENDIX 11 \\ DC REGREBSION BTATIBTIC8 FROX 8AB PROGRAK ( APPENDII 13 )
}

BENBOR 2

$\begin{array}{llllll}A 2 & \mathrm{B2} & 82 \mathrm{~A} & 82 \mathrm{B2} & \mathrm{CA} 2 \mathrm{B2} & \text { 8IG_RND2 }\end{array}$

$\operatorname{VAR}(\mathrm{A}) \mathrm{VAR}(\mathrm{B2}) \quad \operatorname{COV}(\mathrm{A2}, \mathrm{B2})$ REBTDOAL $\sigma$

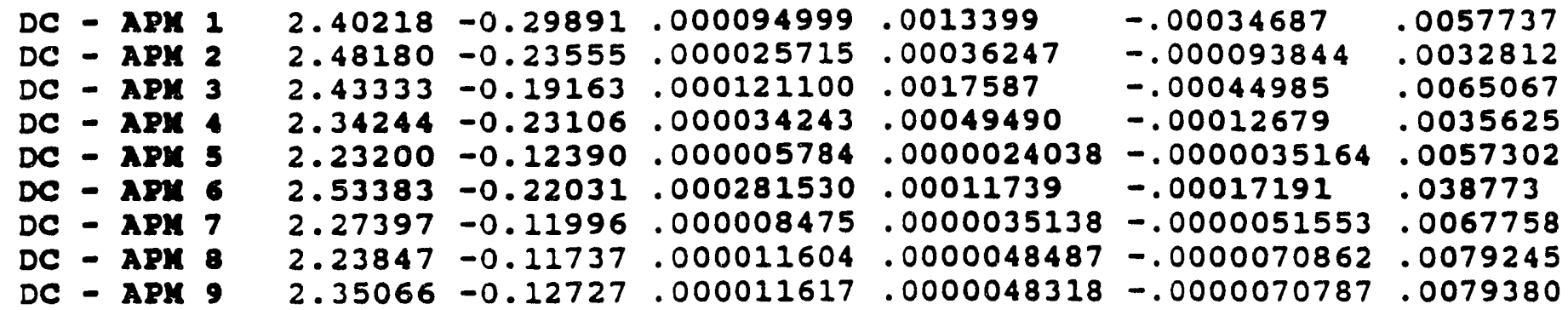

SEYBOR 6

$\begin{array}{llllll}\text { A6 } & B 6 & 82 \lambda 6 & 8286 & \text { CA6B6 } & \text { 8IG_RND6 }\end{array}$

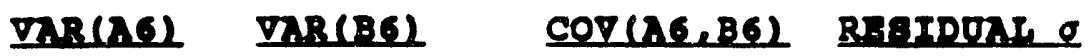

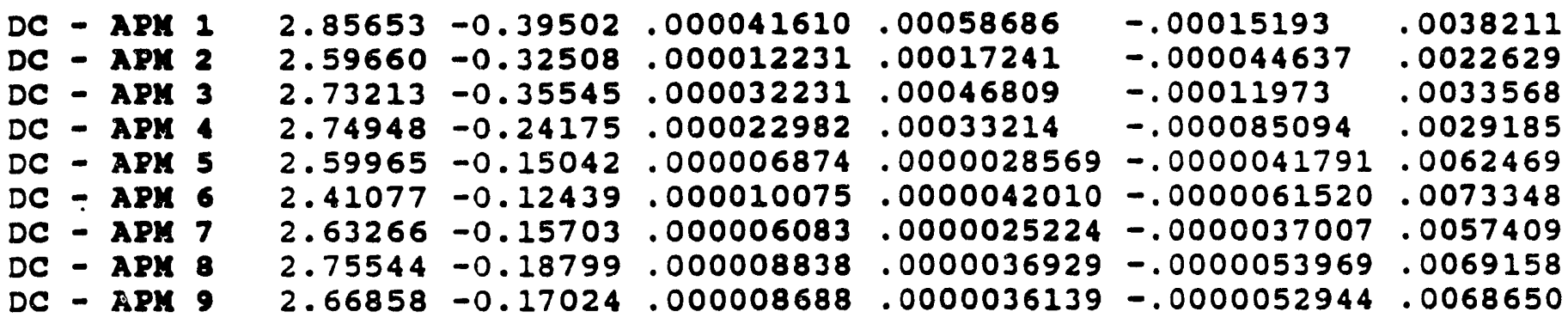


WSRC-TR-92-259

May 29, 1992

Task 92-053-1

Revision 0

Statistical Analysis of Test Data for APM Rod Issue Page 82 of 87

\section{APPENDII 12}

AC REGRB88ION 8TATIBTIC8 FROM BA8 PROGRAK ( APPENDIX 13)

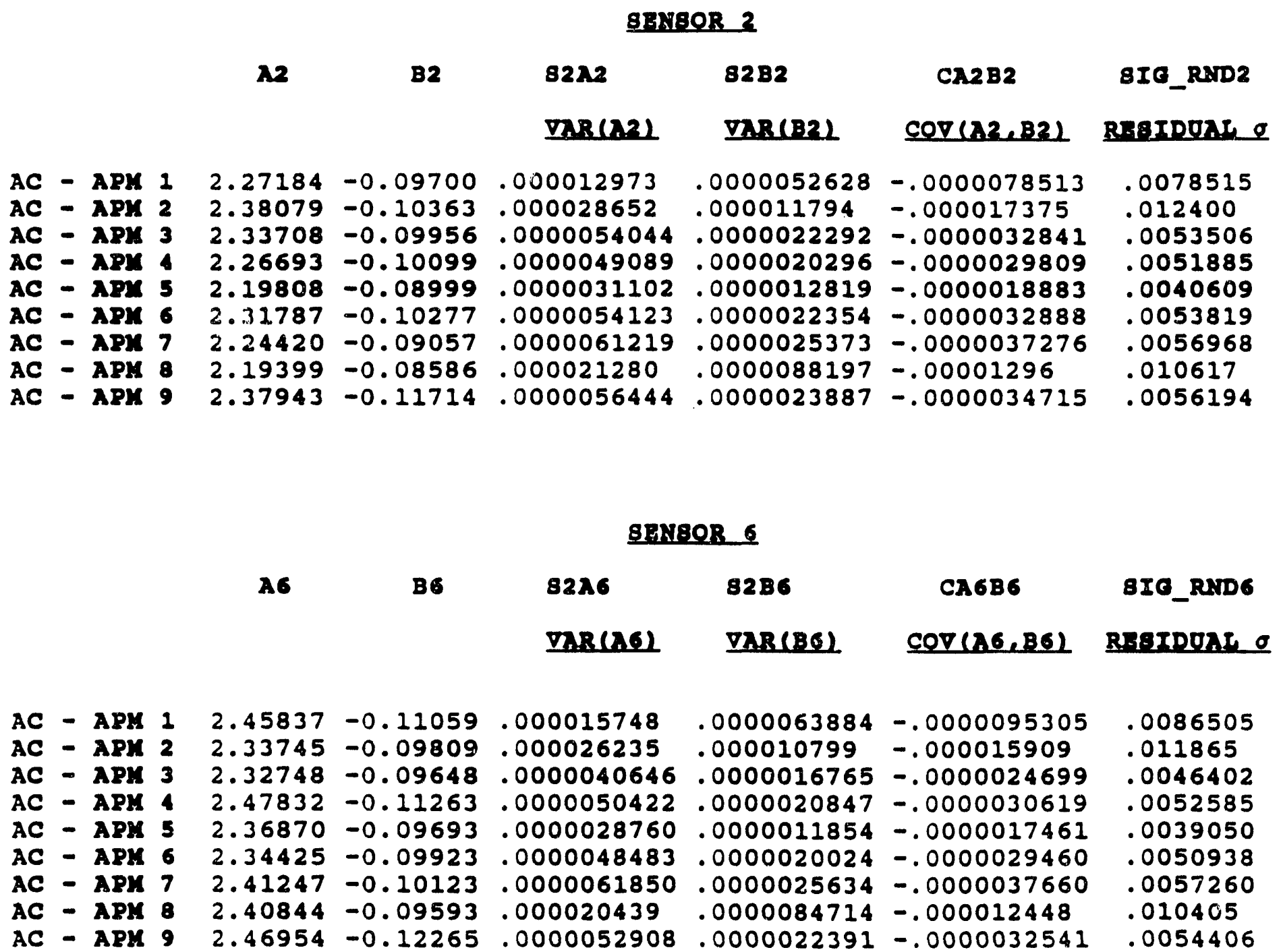


DATA DO;

INFILE 'D: \TOM \ROD1123.TXT';

INPUT A1-A13;

$Y=A 11 ; \quad$ * FOR SENSOR 2 (MV);

$X=A 10$; * WATTS/ GRAM;

$\mathrm{X} 2=\mathrm{X} * \mathrm{X}$;

$W=1 ; \quad \star \star \star \star$ FOR UNWEIGHTED REGRESSION;

: DROP AI-A9 A13;

DATA DO;SET DO;

IF $X$ LE 0.0001 THEN DELETE;

PROC PRINT DATA $=D O(O B S=50)$ UNIFORM;

PROC REG DATA=DO OUTEST=COEFF COVOUT;

MODEL $Y=X X 2 /$ COVB NOINT;

WEIGHT $W$;

DATA DI; SET COEFF; $\mathrm{N}=1$;

IF _TYPE_ = 'PARMS' THEN DO;

MSE $=$ =RMSE_ $\star 2$;

$\mathrm{B1}=\mathrm{X}:$

$\mathrm{B} 2=\mathrm{X} 2$; OUTPUT ;

END:

DATA D3; SET COEFF; $N=1$;

IF _TYPE_='COV' AND _NAME_='X' THEN DO; $\mathrm{V}-\overline{\mathrm{B}} 1=\mathrm{X}$;

END;

$C_{-}^{-12}=X 2$; OUTPUT;

DATA D4; SET COEFF; $\mathrm{N}=1$;

IF_TYPE_ $=\prime$ COV' AND_NAME_ $=\prime X 2^{\prime}$ THEN DO;

- END;

$$
\mathrm{V}_{-} \mathrm{B2}=\mathrm{X} 2 \text {; OUTPUT: }
$$

DATA DZ;SET DO;N=1;IF_N_=1 THEN OUTPUT; KEEP $N$;

DATA STATS2; MERGE DI D3-D4 DZ;BY N;

DROP BI V_BI V_B2 C_12 MSE;

$I D=1$;

$\mathrm{A} 2=\mathrm{B} 1$;

$\mathrm{B} 2=\mathrm{B2}$;

$\mathrm{S} 2 \mathrm{~A} 2=\mathrm{V} \_\mathrm{BI}$; 


\section{APPENDIX 13}

( CONTINOED )

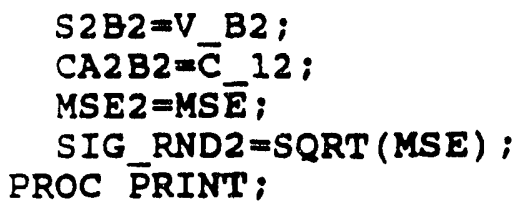




\section{APPENDIX 13}

( CONTINOED )

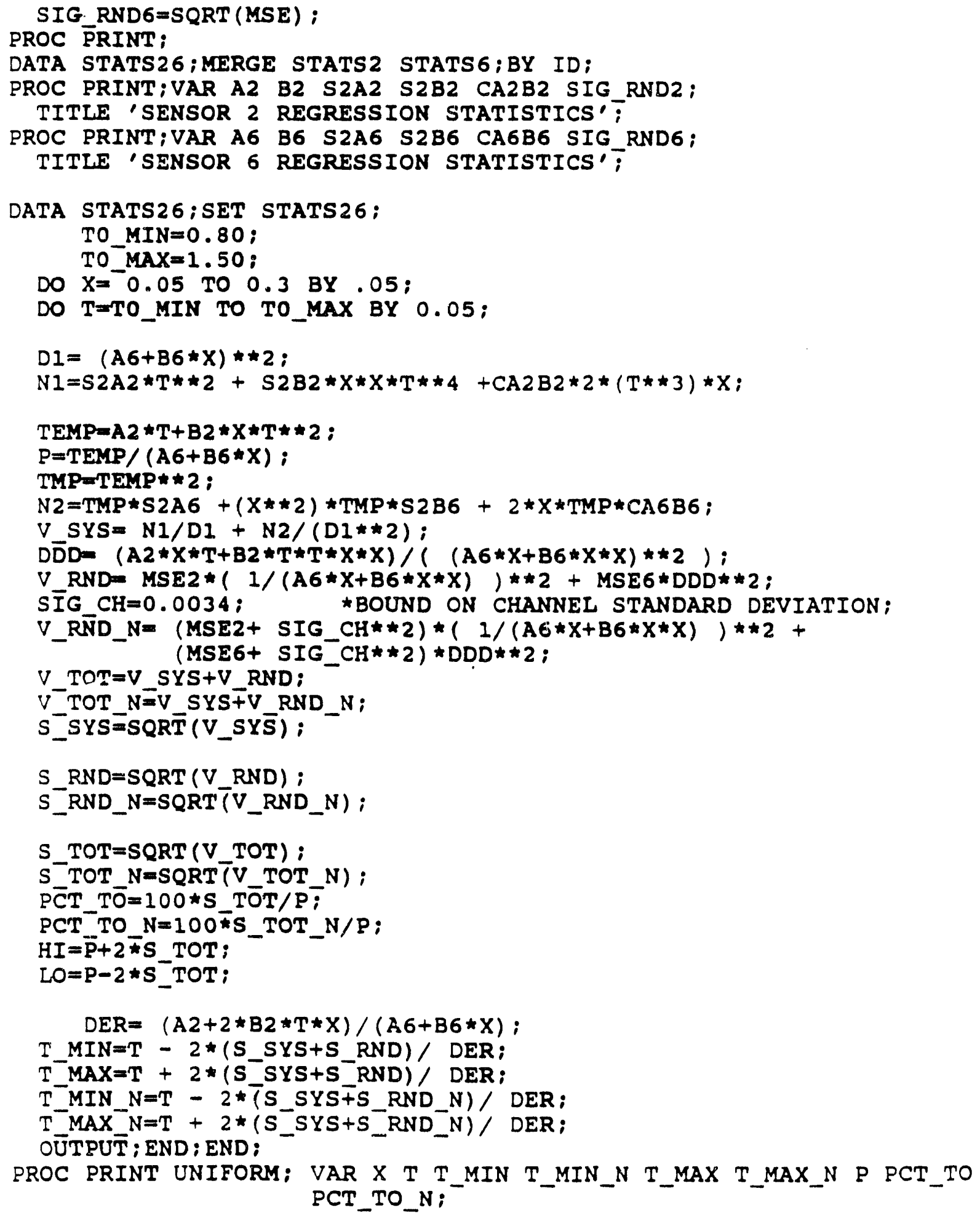




\section{APPENTIX 13}

( CONTINOED )

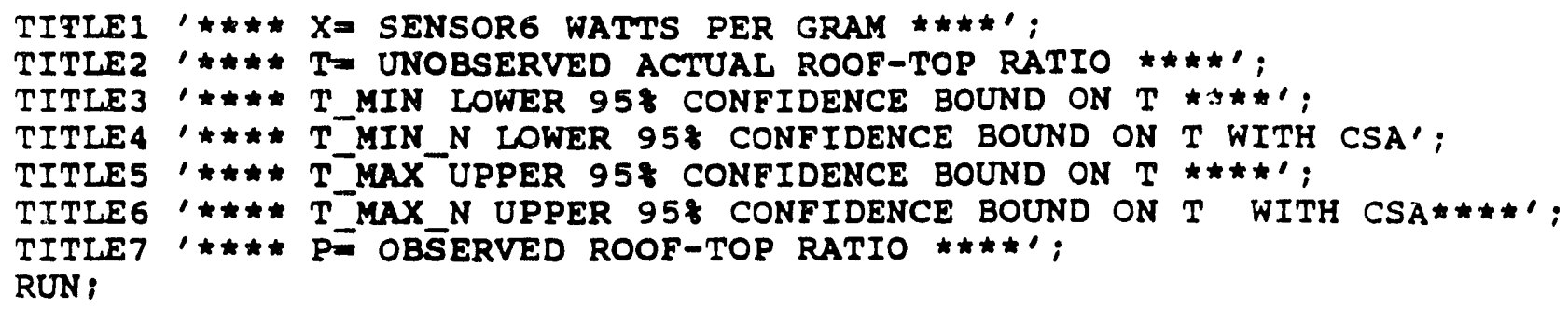

RUN ; 
WSRC-TR-92-259

May 29, 1992

Task 92-053-1

Revision 0

Statistical Analysis of Test Data for APM Rod Issue Page 87 of 87

\section{Appendix 14: The Method of Propagation of Errors}

The description of the method of propagation of errors provided in this appendix is from [3]. Assume that the random variables $x_{1}, x_{2}, \ldots, x_{n}$ have means $\mu_{1}, \mu_{2}, \ldots$, $\mu_{n}$ and variances $\sigma_{11}, \sigma_{22}, \ldots, \sigma_{n n}$. Let the covariance between $x_{i}$ and $x_{j}$ be given by $\sigma_{i j}$ and let $u$ represent a derived value that may be expressed a function $f$ of the $x$ 's:

$$
u=f\left(x_{1}, x_{2}, \ldots, x_{n}\right)
$$

The variance of $u, V(u)$, is approximated by

$$
\begin{aligned}
V(u) \approx\left(\partial f / \partial x_{1}\right)^{2} & \sigma_{11}+\left(\partial f / \partial x_{2}\right)^{2} \sigma_{22}+\ldots+\left(\partial f / \partial x_{n}\right)^{2} \sigma_{n n} \\
& +2\left(\partial f / \partial x_{1}\right)\left(\partial t / \partial x_{2}\right) \sigma_{12}+\ldots+2\left(\partial f / \partial x_{1}\right)\left(\partial f / \partial x_{n}\right) \sigma_{1 n} \\
& +\ldots+ \\
& +2\left(\partial f / \partial x_{n-1}\right)\left(\partial f / \partial x_{n}\right) \sigma_{n-1, n}
\end{aligned}
$$

The partial derivatives, $(\partial f / \partial \cdot)$, are evaluated at the mean values of the random variables. 

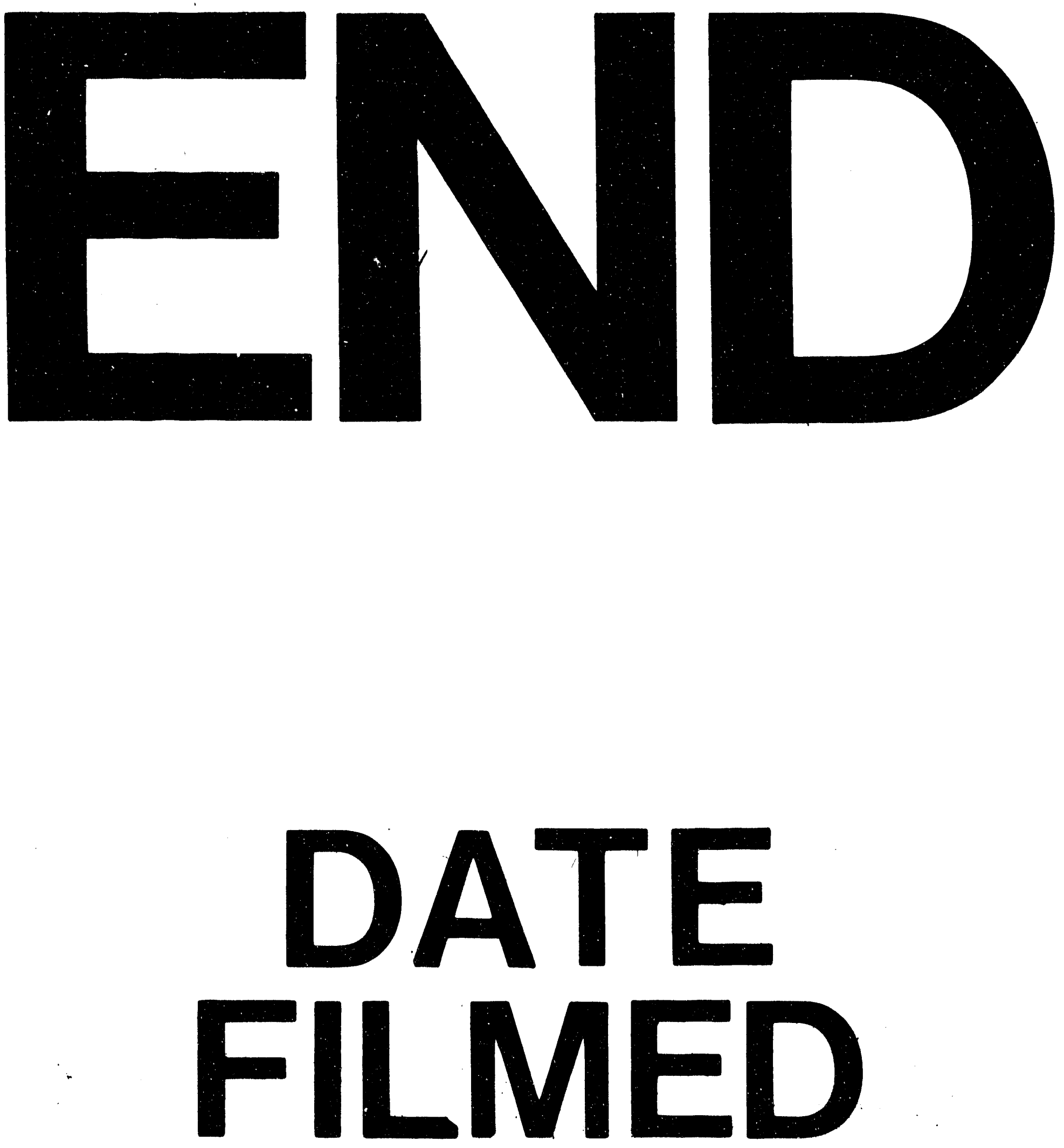

I

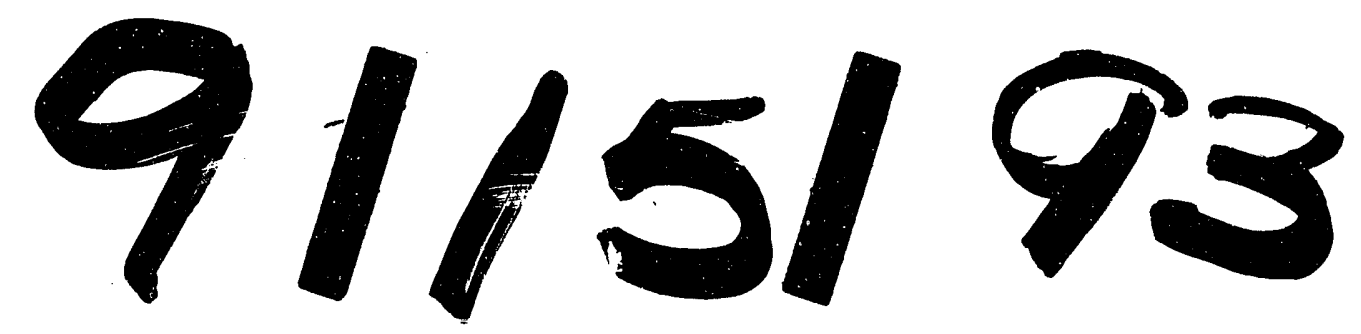


